\title{
Ostracods (Crustacea) of the Early-Middle Permian (Cisarulian-Guadalupian) from Central Thailand (Indochina Block): Part II, Orders Podocopida, Platycopida and Myodocopida
}

Anisong CHITNARIN

School of Geotechnology, Institute of Engineering, Suranaree University of Technology, 111 University Avenue, Mueang District, Nakhon Ratchasima Province, 30000 (Thailand) anisong@sut.ac.th (corresponding author)

Sylvie CRASQUIN CR2P (MNHN, CNRS, UPMC-Paris 6, Sorbonne Universités), 4 place Jussieu, T.46-56, E.5, case 104, 75252 Paris cedex 05 (France) sylvie.crasquin@upmc.fr

Marie-Béatrice FOREL Independent researcher mbforel@yahoo.fr

Prachya TEPNARONG School of Geotechnology, Institute of Engineering, Suranaree University of Technology, 111 University Avenue, Mueang District, Nakhon Ratchasima Province, 30000 (Thailand) prachya@sut.ac.th

Published on 29 December 2017

KEY WORDS

Ostracods,

Podocopida,

Platycopida

Early-Middledocopida,

Thailand,

Indochina Block,

new synonyms,

new species.
Chitnarin A., Crasquin S., Forel M.-B. \& Tepnarong P. 2017. - Ostracods (Crustacea) of the Early-Middle Permian (Cisarulian-Guadalupian) from Central Thailand (Indochina Block): Part II, Orders Podocopida, Platycopida and Myodocopida. Geodiversitas 39 (4): 651-690. https://doi.org/10.5252/g2017n4a1

\section{ABSTRACT}

In the present contribution, we report abundant Early to Middle Permian (Cisarulian-Guadalupian) marine ostracods of the Indochina block from Central Thailand. Twelve localities in the Loei, Phetchabun, and Nakhon Sawan-Lopburi areas were investigated and limestone samples were collected. Out of 135 samples collected, 82 yielded abundant and nicely preserved ostracods. In a first paper, 39 species of 20 genera belonging to Order Palaeocopida were already described. Here is the second part of the ostracod fauna description with 91 species of the orders Podocopida, Platycopida and Myodocopida. Fourteen species are new and described here: Bairdia khaokanaensis Chitnarin, n. sp., Bairdia incisedorsa Chitnarin, n. sp. Bairdia fontainei Chitnarin, n. sp., Bairdia songthami Chitnarin, n. sp., Bairdia grotei Chitnarin, n. sp., Petasobairdia campbelli Chitnarin, n. sp., Fabalicypris hathaithipae Chitnarin, n. sp., Silenites sureeae Chitnarin, n. sp., Acratia chongpani Chitnarin, n. sp., Acratia mongkoli Chitnarin, n. sp., Liuzhinia naramasei Chitnarin, n. sp., Baschkirina ruchae Chitnarin, n. sp., Basslerella wipanuae Chitnarin, n. sp., Basslerella naresi Chitnarin, n. sp. An analysis of the palaeogeographic affinities of the recovered ostracods shows close relationships with those found from South China Block on the Paleo-Tethys realm. 


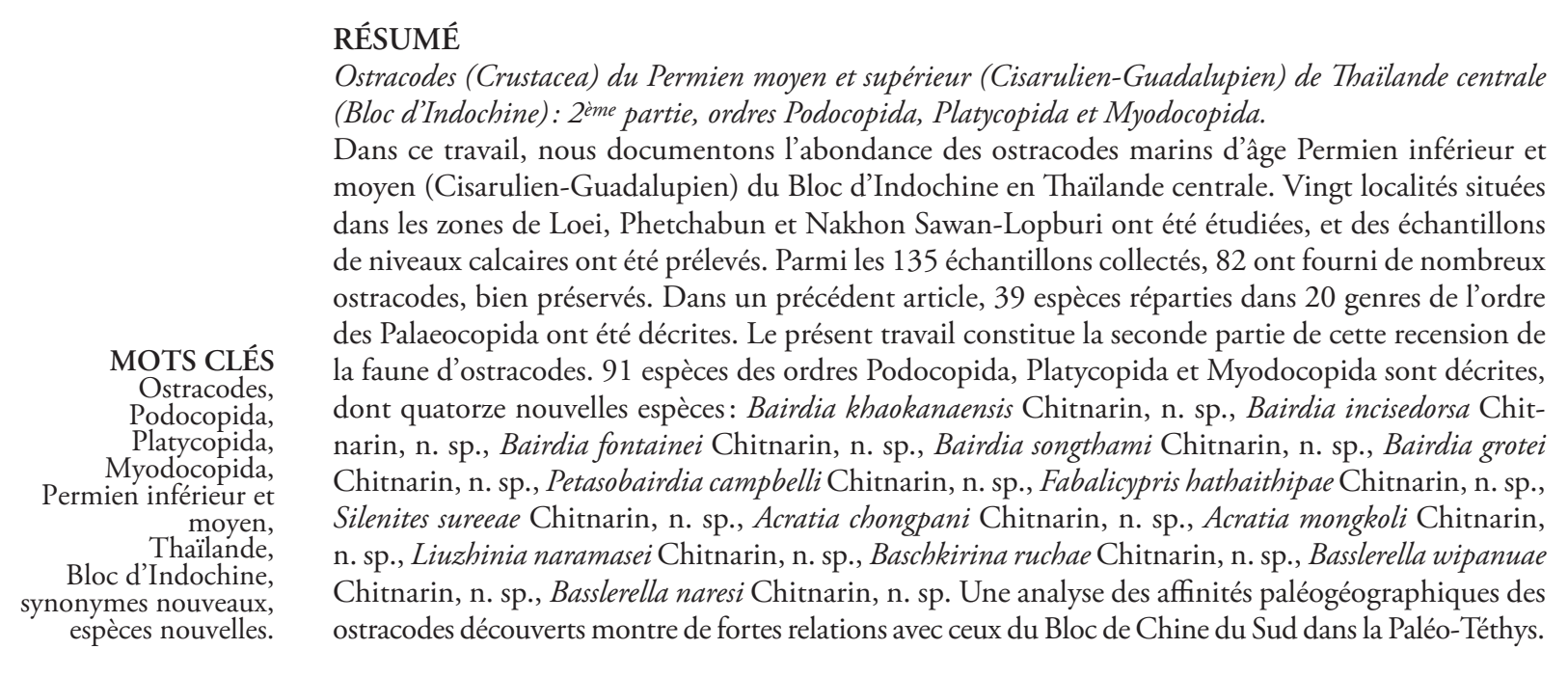

\section{INTRODUCTION}

This article is the second part of the research papers concerning the Early-Middle Permian ostracods discovered in central Thailand. The previous article was devoted to the Order Palaeocopida (Chitnarin et al. 2012); thus, this paper is focuses on three other orders, Podocopida, Platycopida and Myodocopida.

Permian palaeontology and micropalaeontology of Thailand (the mainland of Southeast Asia) has been investigated by many researchers (e.g. Dawson \& Racey 1993; Pérez-Huerta et al. 2007; Metcalfe \& Sone 2008; Sone et al. 2009; Burrett et al. 2014; Ketwetsuriya et al. 2016). The objectives were age calibration, palaeoecological reconstruction, palaeogeographic and palaeobiogeographic implications of this area. Ostracods (Crustacea) are accepted to be one of the most efficient palaeoecological index (e.g. Melnyk \& Maddocks 1988; Crasquin-Soleau \& Baud 1998; Crasquin-Soleau et al. 1999) though data on this group are rare in Thailand. So far, three papers have been published; one on the late Middle Permian (Chitnarin et al. 2008) and another one on Early-Middle Permian (Chitnarin et al. 2012; Burrett et al. 2014), both from central Thailand.

\section{GEOLOGICAL SETTING}

Details of study areas and sections were described in Chitnarin et al. (2012, with references therein). Twelve localities were investigated in three areas namely the Loei, the Phetchabun and the Nakhon Sawan-Lopburi (Figs 1-2). All samples were collected from limestones of Nam Maholan, Pha Nok Khao and Tak Fa Formations (Saraburi Group) which range from early Early Permian to late Middle Permian (Asselian to Capitanian age; Table 1). Please note that the Takli section in Nakhon Sawan-Lopburi area indicated as Middle Permian in Chitnarin et al. (2012) is re-assigned to Early Permian (Artinskian) due to the recent discovery of fusulinids (Pamirina sp. and Pseudofusulina sp.; M. Sone pers. comm. 2016).

\section{MATERIAL AND METHODS}

135 limestone samples were collected and processed by hot acetolysis (Lethiers \& Crasquin-Soleau 1988; Crasquin-Soleau et al. 2005); 82 yielded ostracods. 130 species have been recovered. The fauna belonging to Order Palaeocopida are published (Chitnarin et al. 2012). Therefore, Subclass Podocopa, Order Podocopida (Bairdiidae, Acratiidae, Sigilloidae, Geroiidae, Cytherideidae), Order Platycopida (Cavellinidae) and Subclass Myodocopa, Order Halocyprida (Polycopidae) in this article include 91 species belonging to 18 genera. Fourteen species are newly described. Due to the availability of specimens, many species are left in open nomenclature and are not listed in the systematic part except when needed. We nevertheless figured all the material to allow future studies ans comparisons. All specimens are deposited in Suranaree University of Technology collections (numbers SUT-09-2xxx).

$\begin{array}{ll}\text { ABBREVIATIONS } \\ \text { L } & \text { length; } \\ \text { H } & \text { height; } \\ \text { W } & \text { width; } \\ \text { AB } & \text { anterior border; } \\ \text { VB } & \text { ventral border; } \\ \text { PB } & \text { posterior border; } \\ \text { DB } & \text { dorsal border; } \\ \text { LV } & \text { left valve; } \\ \text { RV } & \text { right valve; } \\ \text { AVB } & \text { anteroventral border; } \\ \text { PVB } & \text { posteroventral border; } \\ \text { ADB } & \text { anterodorsal border; } \\ \text { PDB } & \text { posterodorsal border. }\end{array}$

The classification used in this paper follows Horne et al. (2002) for the Quaternary and living forms and Moore (1961) and Becker (2002) for extinct ones. All the sections are located in Central Thailand. 


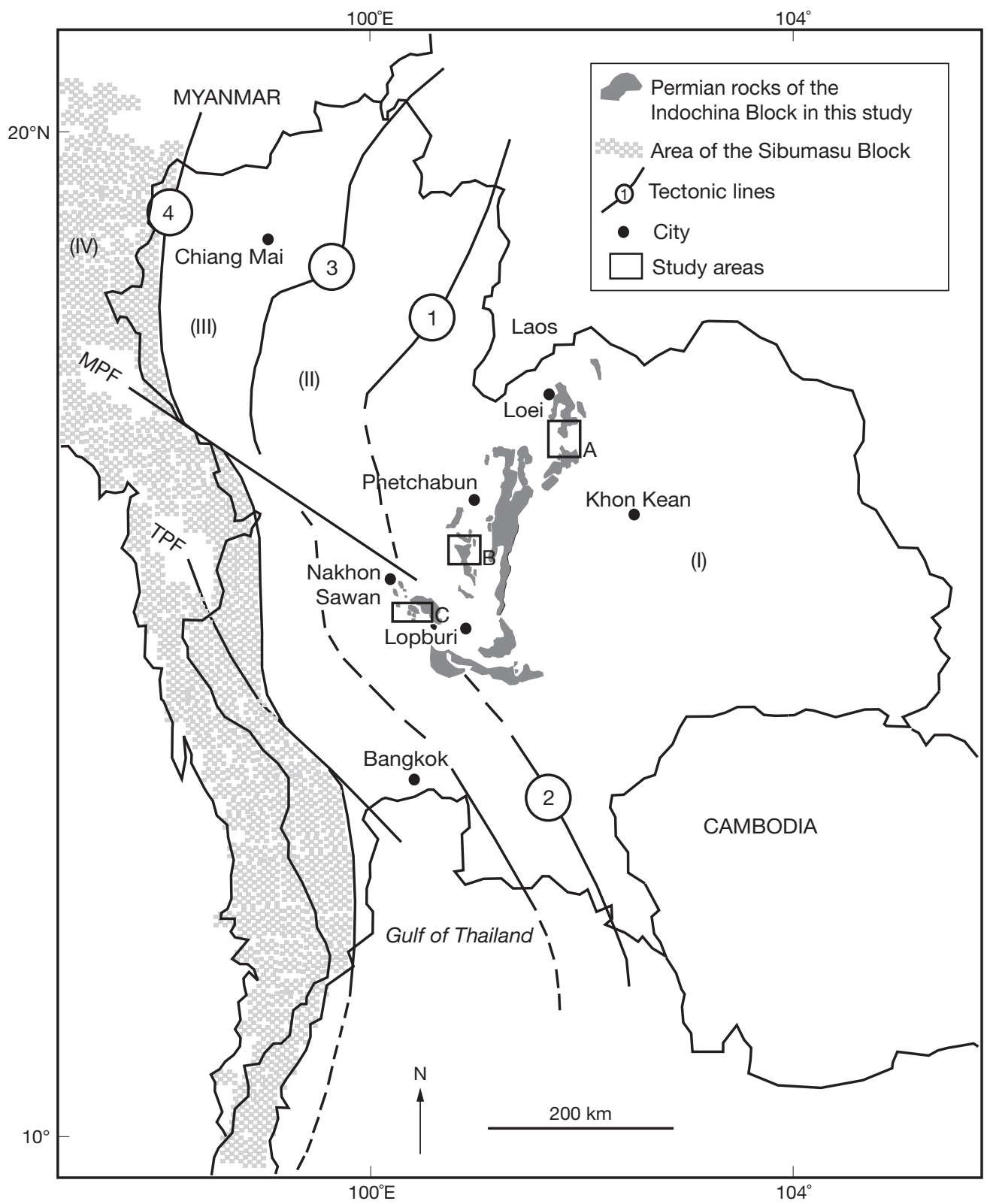

FIG. 1. - Geotectonic map of mainland Thailand, with distribution of Permian rocks of the Indochina block relevant to this study and the studied areas: A, Loei area; B, Phetchabun area; C, Nakhon Sawn-Lopburi area; I, Indochina block; II, Sukhothai zone; III, Intanon zone; IV, Sibumasu block; MPF, Mae Ping Fault; TPF, Three Pagodas Fault (Chitnarin et al. 2012).

\section{SYSTEMATIC PALAEONTOLOGY}

Class OSTRACODA Latreille, 1802

Subclass PODOCOPA Sars, 1866

Order PODOCOPIDA Sars, 1866

Suborder PODOCOPINA Sars, 1866

Superfamily BAIRDIODEA Sars, 1888

Family BAIRDIIDAE Sars, 1888

Genus Bairdia McCoy, 1844

TYPe SPECIES. - Bairdia curta McCoy, 1844, by subsequently designated by Ulrich \& Bassler (1923: 320).
Bairdia khaokanaensis Chitnarin, n. sp.

(Figs 3A-D; 4)

TYPE MATERIAL. - Holotype: one complete carapace (SUT-092226; Fig. 3A); paratypes: two complete carapaces (SUT-09-2223; Fig. 3B and SUT-09-2231; Fig. 3C).

MATERIAL EXAMINED. - One damaged and one complete carapaces.

DiAGNOSIS. - Species of Bairdia with long carapace, ventral outline broadly arched, AB nearly vertical, $A V B$ and PVB of both valves long and convex, VB slightly concave, short and convex DB.

Eтymology. - From the type locality, the Khao Kana section. 


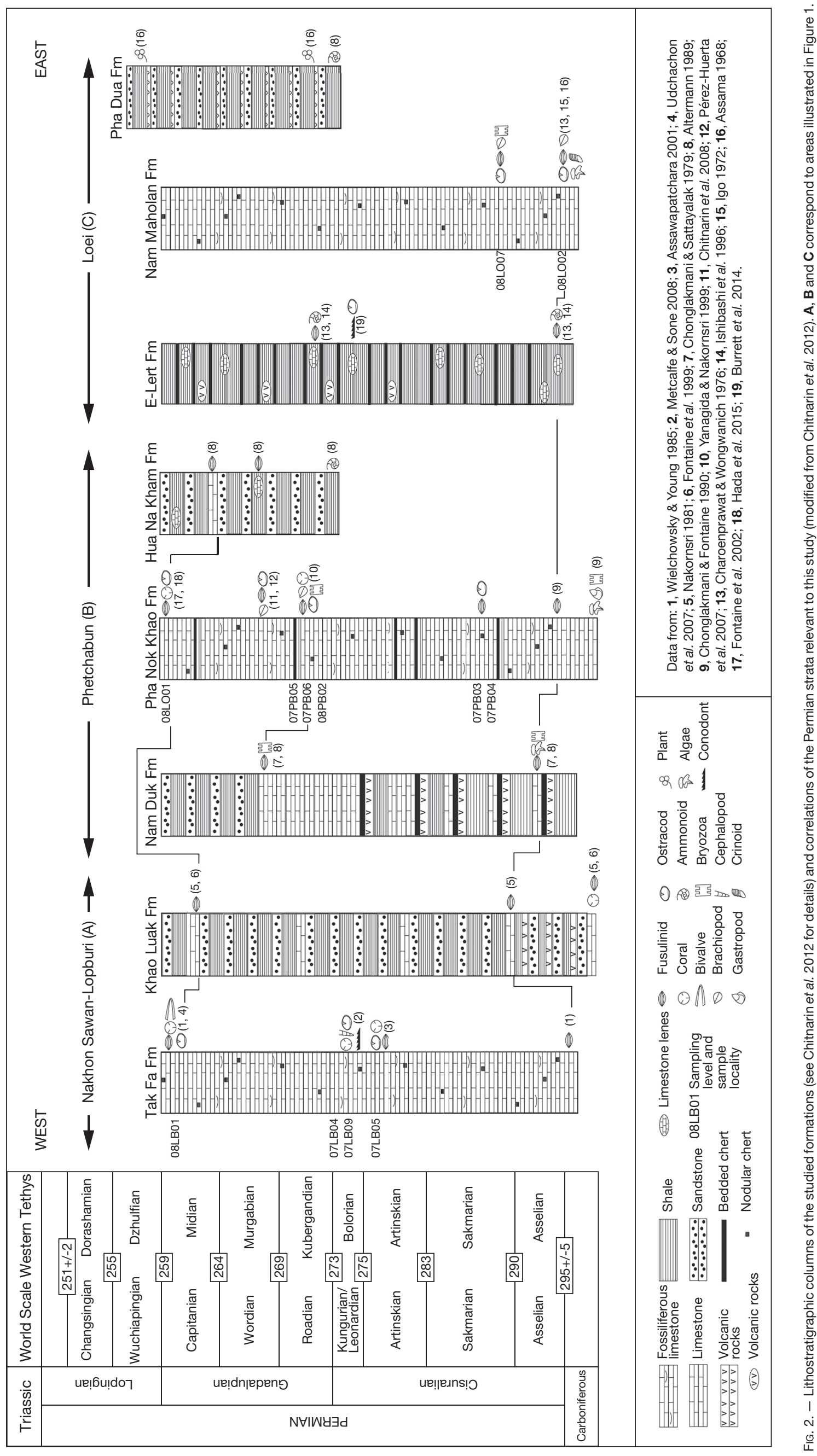


TABLE 1. - Information on studied sections (slightly modified from Chitnarin et al. 2012). Abbreviation: Thick., thickness (in metres).

\begin{tabular}{|c|c|c|c|c|c|c|c|c|c|c|}
\hline Area & $\begin{array}{l}\text { Section } \\
\text { number }\end{array}$ & $\begin{array}{l}\text { Section } \\
\text { name }\end{array}$ & Formation & Age & $\begin{array}{l}\text { Geographic } \\
\text { position }\end{array}$ & $\begin{array}{l}\text { Provincial } \\
\text { boundary }\end{array}$ & Thick. & Lithology & $\begin{array}{l}\text { Limestone } \\
\text { classification }\end{array}$ & $\begin{array}{l}\text { Nb of } \\
\text { samples \& } \\
\text { sample no. }\end{array}$ \\
\hline \multirow{3}{*}{ 'ָ } & 08LO01 & $\begin{array}{l}\text { Khao Tham } \\
\text { Yai }\end{array}$ & $\begin{array}{l}\text { Pha Nok } \\
\text { Khao }\end{array}$ & Capitanian & $\begin{array}{l}16^{\circ} 56^{\prime} 22^{\prime \prime} \mathrm{N}, \\
101^{\circ} 23^{\prime} 37^{\prime \prime} \mathrm{E}\end{array}$ & $\begin{array}{l}\text { Nam Nao } \\
\text { district, } \\
\text { Phetchabun }\end{array}$ & 2 & $\begin{array}{l}\text { Dark gray, } \\
\text { argillaceous } \\
\text { limestone }\end{array}$ & $\begin{array}{l}\text { wackestone- } \\
\text { packstone }\end{array}$ & $\begin{array}{l}4 \\
(08 L O 01-1 \\
\text { to } 4)\end{array}$ \\
\hline & 08LO02 & $\begin{array}{l}\text { Tham Nam } \\
\text { Maholan }\end{array}$ & $\begin{array}{l}\text { Nam } \\
\text { Maholan }\end{array}$ & Asselian & $\begin{array}{l}17^{\circ} 066^{\prime} 23^{\prime \prime} \mathrm{N}, \\
101^{\circ} 52^{\prime} 48^{\prime \prime} \mathrm{E}\end{array}$ & $\begin{array}{l}\text { Wang Saphung } \\
\text { district, Loei }\end{array}$ & 40 & $\begin{array}{l}\text { light gray, } \\
\text { micritic, } \\
\text { limestones }\end{array}$ & $\begin{array}{l}\text { wackestone- } \\
\text { packstone }\end{array}$ & $\begin{array}{l}12 \\
(08 \mathrm{LO} 02-1 \\
\text { to } 12)\end{array}$ \\
\hline & 08LO07 & $\begin{array}{l}\text { Sak Chai } \\
\text { Quarry }\end{array}$ & $\begin{array}{l}\text { Pha Nok } \\
\text { Khao }\end{array}$ & $\begin{array}{l}\text { Asselian- } \\
\text { Sakmarian }\end{array}$ & $\begin{array}{l}16^{\circ} 35^{\prime} 07^{\prime \prime} \mathrm{N}, \\
101^{\circ} 52^{\prime} 38^{\prime \prime} \mathrm{E}\end{array}$ & $\begin{array}{l}\text { Khon San } \\
\text { district, } \\
\text { Chaiyaphum }\end{array}$ & 90 & $\begin{array}{l}\text { drak gray, } \\
\text { argillaceous, } \\
\text { limestones }\end{array}$ & $\begin{array}{l}\text { wackestone- } \\
\text { packstone }\end{array}$ & $\begin{array}{l}12 \\
(08 L O 07-1 \\
\text { to 12) }\end{array}$ \\
\hline \multirow{5}{*}{$\begin{array}{l}\frac{5}{3} \\
\frac{0}{\pi} \\
\frac{5}{0} \\
\frac{1}{0} \\
\frac{c}{2}\end{array}$} & 07PB03 & Khao Kana & $\begin{array}{l}\text { Pha Nok } \\
\text { Khao }\end{array}$ & $\begin{array}{l}\text { Asselian- } \\
\text { Sakmarian }\end{array}$ & $\begin{array}{l}16^{\circ} 04^{\prime} 12^{\prime \prime} \mathrm{N}, \\
101^{\circ} 54^{\prime} 20 ” \mathrm{E}\end{array}$ & $\begin{array}{l}\text { Chon Dan } \\
\text { district, } \\
\text { Phetchabun }\end{array}$ & 15 & $\begin{array}{l}\text { dark gray, } \\
\text { micritic } \\
\text { limestones }\end{array}$ & wackestone & $\begin{array}{l}7 \\
(07 P B 03-1 \\
\text { to } 7)\end{array}$ \\
\hline & 07PB04 & Nong Phai & $\begin{array}{l}\text { Pha Nok } \\
\text { Khao }\end{array}$ & $\begin{array}{l}\text { Asselian- } \\
\text { Sakmarian }\end{array}$ & $\begin{array}{l}16^{\circ} 01^{\prime} 06^{\prime \prime} \mathrm{N}, \\
101^{\circ} 58^{\prime} 59 " \mathrm{E}\end{array}$ & $\begin{array}{l}\text { Nong Phai } \\
\text { district, } \\
\text { Phetchabun }\end{array}$ & 30 & $\begin{array}{l}\text { gray-light to } \\
\text { gray, micritic } \\
\text { limestones }\end{array}$ & $\begin{array}{l}\text { wackestone- } \\
\text { packstone }\end{array}$ & $\begin{array}{l}7 \\
(07 P B 04-1 \\
\text { to } 7)\end{array}$ \\
\hline & 07PB05 & $\begin{array}{l}\text { Ban Naen } \\
\text { Sawan I }\end{array}$ & Tak Fa & Roadian? & $\begin{array}{l}15^{\circ} 54^{\prime} 11^{\prime \prime} \mathrm{N}, \\
100^{\circ} 53^{\prime} 36 " \mathrm{E}\end{array}$ & $\begin{array}{l}\text { Bung Sam } \\
\text { Phan district, } \\
\text { Phetchabun }\end{array}$ & 30 & $\begin{array}{l}\text { dark gray, } \\
\text { argillaceous } \\
\text { limestones }\end{array}$ & $\begin{array}{l}\text { mudstone- } \\
\text { wackestone }\end{array}$ & $\begin{array}{l}7 \\
(07 P B 05-1 \\
\text { to } 7)\end{array}$ \\
\hline & $\begin{array}{l}\text { 07PB06- } \\
\text { 07PB08 }\end{array}$ & $\begin{array}{l}\text { Ban Naen } \\
\text { Sawan II }\end{array}$ & Tak Fa & Roadian? & $\begin{array}{l}15^{\circ} 55^{\prime} 08^{\prime \prime} \mathrm{N}, \\
100^{\circ} 55^{\prime} 46^{\prime \prime} \mathrm{E}\end{array}$ & $\begin{array}{l}\text { Bung Sam } \\
\text { Phan district, } \\
\text { Phetchabun }\end{array}$ & 90 & $\begin{array}{l}\text { light gray, } \\
\text { micritic- } \\
\text { crystalline } \\
\text { limestones }\end{array}$ & $\begin{array}{l}\text { mudstone- } \\
\text { wackestone }\end{array}$ & $\begin{array}{l}21 \\
\text { (07PB06-1 } \\
\text { to 8), } \\
\text { (07PB07-1 } \\
\text { to 4), } \\
\text { (07PB08-1 } \\
\text { to 9) }\end{array}$ \\
\hline & $\begin{array}{l}\text { 08PB01- } \\
\text { 08PB03 }\end{array}$ & $\begin{array}{l}\text { Phu Phra } \\
\text { That }\end{array}$ & Tak Fa & Roadian? & $\begin{array}{l}15^{\circ} 58^{\prime} 22^{\prime \prime} \mathrm{N}, \\
100^{\circ} 52^{\prime} 21^{\prime \prime} \mathrm{E}\end{array}$ & $\begin{array}{l}\text { Chon Dan } \\
\text { district, } \\
\text { Phetchabun }\end{array}$ & 70 & $\begin{array}{l}\text { dark gray- } \\
\text { gray, micritic } \\
\text { limestones }\end{array}$ & $\begin{array}{l}\text { wackestone- } \\
\text { packstone }\end{array}$ & $\begin{array}{l}19 \\
\text { (08PB02-1 } \\
\text { to 13), } \\
\text { (08PB03-1 } \\
\text { to } 6)\end{array}$ \\
\hline \multirow{4}{*}{ 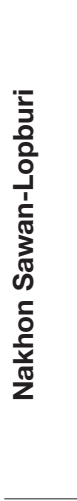 } & 07LB04 & $\begin{array}{l}\text { Phu Lam } \\
\text { Yai }\end{array}$ & Tak Fa & Kungurian? & $\begin{array}{l}15^{\circ} 20^{\prime} 21^{\prime \prime} \mathrm{N}, \\
100^{\circ} 36^{\prime} 15^{\prime \prime} \mathrm{E}\end{array}$ & $\begin{array}{l}\text { Tak Fa district, } \\
\text { Nakhon Sawan }\end{array}$ & 30 & $\begin{array}{l}\text { gray, micritic } \\
\text { limestone }\end{array}$ & $\begin{array}{l}\text { mudstone- } \\
\text { wackestone }\end{array}$ & $\begin{array}{l}18 \\
(07 L B 04-1 \\
\text { to } 18)\end{array}$ \\
\hline & 07LB05 & Ta Kli & Tak Fa & Artinskian & $\begin{array}{l}15^{\circ} 19^{\prime} 05^{\prime \prime} \mathrm{N}, \\
100^{\circ} 22^{\prime} 46^{\prime \prime} \mathrm{E}\end{array}$ & $\begin{array}{l}\text { Ta Kli District, } \\
\text { Nakhon Sawan }\end{array}$ & 50 & $\begin{array}{l}\text { dark gray, } \\
\text { argillaceous } \\
\text { limestone }\end{array}$ & $\begin{array}{l}\text { wackstone- } \\
\text { packstone }\end{array}$ & $\begin{array}{l}20 \text { (07LB05-1 } \\
\text { to } 5) \text {, } \\
\text { (07LB05-A1 } \\
\text { to A3, B1 to } \\
\text { B3, D1 to D3) }\end{array}$ \\
\hline & 07LB09 & $\begin{array}{l}\text { Khao Phu } \\
\text { Chongkho }\end{array}$ & Tak Fa & Kungurian? & $\begin{array}{l}15^{\circ} 22^{\prime} 45^{\prime \prime} \mathrm{N}, \\
100^{\circ} 35^{\prime} 12^{\prime \prime} \mathrm{E}\end{array}$ & $\begin{array}{l}\text { Tak Fa District, } \\
\text { Nakhon Sawan }\end{array}$ & 1 & $\begin{array}{l}\text { light gray, } \\
\text { micritic } \\
\text { limestone }\end{array}$ & $\begin{array}{l}\text { wackstone- } \\
\text { packstone }\end{array}$ & $\begin{array}{l}2 \\
\text { (07LB09-1 } \\
\text { to 2) }\end{array}$ \\
\hline & 08LB01 & $\begin{array}{l}\text { Khao } \\
\text { Somphot }\end{array}$ & Tak Fa & Capitanian & $\begin{array}{l}15^{\circ} 11^{\prime} 16^{\prime \prime} \mathrm{N}, \\
101^{\circ} 31^{\prime} 10^{\prime \prime} \mathrm{E}\end{array}$ & $\begin{array}{l}\text { Lam Sonthi } \\
\text { District, Lopburi }\end{array}$ & 15 & $\begin{array}{l}\text { dark gray, } \\
\text { micritic, } \\
\text { argillaceous } \\
\text { limestones }\end{array}$ & $\begin{array}{l}\text { wackstone- } \\
\text { packstone }\end{array}$ & $\begin{array}{l}6 \\
(08 L B 01-1 \\
\text { to } 6)\end{array}$ \\
\hline
\end{tabular}

DimENSIONS. $-\mathrm{H}=0.23-0.57 \mathrm{~mm}, \mathrm{~L}=0.51-1.28 \mathrm{~mm}, \mathrm{H} / \mathrm{L}=$ 0.43-0.47, Holotype: $\mathrm{H}=0.563 \mathrm{~mm}, \mathrm{~L}=1.22 \mathrm{~mm}$; Paratypes: $\mathrm{H}=$ $0.322, \mathrm{~L}=0.726$ (Fig. 4; both paratypes of the same size).

TyPe HORIZON. - Sample 07PB03-3, Khao Kana section, Pha Nok Khao Formation, Sakmarian, Early Permian.

TYPe LOCALITY. - Khao Kana section (160.'12”N, 100 54’20”E), Phetchabun Province, Central Thailand.

OCCURRENCES. - Samples 07PB03-1, 07PB03-3 and 07PB03-5, Khao Kana section, Phetchabun Province, Pha Nok Khao Formation, Early Permian, Central Thailand.

\section{DESCRIPTION}

Carapace long; dorsal outline regularly arched; DB short (30\% of L); ADB nearly straight, angle with $\mathrm{DB}$ is $150^{\circ}$;
$\mathrm{AB}$ round with moderate radius of curvature, maximum convexity located just below mid $\mathrm{H}$; angle between $\mathrm{AB}$ and $\mathrm{ADB}$ is $150^{\circ}$; $\mathrm{AVB}$ long and convex; VB slightly concave; PVB long and convex; posteroventral region flattened; PB round with small radius of curvature, maximum convexity located below mid H; PDB nearly straight, angle with DB of $140^{\circ}$; LV slightly overlaps RV all around the carapace, except at AVB and PVB; maximum $\mathrm{H}$ located at mid $\mathrm{L}$.

\section{REMARKS}

This species can be compared to Bairdia subfusiformis Hamilton, 1942 from the Guadalupian, Middle Permian of Texas (Hamilton 1942) in general outline, but it can be 
TABLE 2. - Stratigraphic distribution of Early-Middle Permian ostracod species from Central Thailand (Indochina block). Dark blocks ( $\mathbf{\square})$ refer to the species described in the present paper, white blocks ( $\square$ ) refer to the species previously described in Chitnarin et al. (2012).

\section{Species}

Fabalicypris sp. B

Acratia ? sp.

Baschkirina cf. huzhouensis Forel, 2010

Microcheilinella sp. B

BAIRDIIDAE indet. sp. A

BAIRDIIDAE indet. sp. B

Knoxiella sp. 2

Bairdia sp. B

Bairdia sp. C

Bairdia sp. L

Petasobairdia sp.

Bairdiacypris sp. C

Microcheilinella sp. C

Microcheilinella shicheni Crasquin, 2010

Polycope sp. D

Bairdia guangxiensis Guan, 1978

Bairdiacypris sp. A

Bairdia incisedorsa Chitnarin, n. sp.

Silenites sp.

Microcheilinella sp. D

Bairdiacypris deloi Bradfield, 1935

?Hollinella $(H.) \mathrm{sp}$.

Shleesha sp.

Reviya subsompongensis Chitnarin, 2008

Bairdia sp. D

Bairdia sp. M

Bairdia sp. I

Fabalicypris sp. C

Bairdiacypris sp. $\mathrm{H}$

Langdaia meesooki Chitnarin, 2012

Paraparchitiidae sp.

Geffenina mariebeatriceae Chitnarin, 2012

Kloedcytherella oertlii Kozur, 1985

Bairdia broutini Crasquin, 2010

Bairdia menardensis Harlton, 1929

Bairdia cf. zhongyingensis Wang, 1978

Bairdia altiarcus Chen, 1958

Bairdia sp. F

Bairdiacypris longirobusta Chen, 1958

Microcheilinella sp. E

Microcoelonella takliensis Chitnarin, 2012

Microcoelonella takfansis Chitnarin, 2012

Microcoelonella? sp.

Knightina? sp.

Shemonaella sp. 2

Samarella sp. 3

Samarella sonei

Eukloedenella? sp.

Knoxiella sp. 1

Samarella sp. 1

Bairdiacypris sp. B

Bairdia sp. H

Petasobairdia campbelli Chitnarin, n. sp.

Petasobairdia subnantongensis Chen, 1987

Lobobairdia ventriconcava (Chen, 1958)

Bairdia urodeloformis Chen, 1987

Bairdia cf. fangnianqiaoi Crasquin, 2010

Bairdia cf. deweveri Crasquin, 2010

Bairdia sp. A

Bairdia fontainei Chitnarin, n. sp.

Bairdia pierrevalentini Crasquin, 2010

Bairdia sp. G

Microcheilinella sp. F

Microcheilinella sp. A

Samarella sp. 2

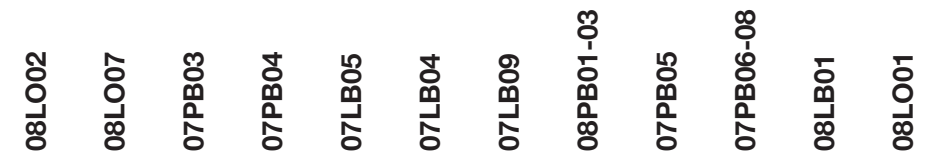

$-$

$-\quad-$

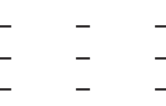

-

$-z^{-}-$




\section{Species}

Knightina cf. ultima (Kozur, 1985)

Shishaella sp.

Fabalicypris sp. A

Bairdiacypris sp. E

Cavellina sp.

Sulcella suprapermiana Kozur, 1985

Bairdia songthami Chitnarin, n. sp.

Bairdia cf. calida Chen, 1958

Bairdiacypris sp. D

Bairdia beedei Ulrich \& Bassler, 1906

Cryptobairdia seminalis (Knight, 1928)

Bairdia cf. B.? mianyangensis Chen, 1982

Bairdia khaokanaensis Chitnarin, n. sp.

Bairdia cf. bassoni Crasquin, 2010

Bairdiacypris sp. G

Kempfina cf. qinglaii (Crasquin, 2008)

Sulcella mesopermiana Kozur, 1985

Hollinella $(H$.$) martensiformis Crasquin, 2010$

Hollinella $(H$.$) herrickana (Girty, 1909)$

Hollinella $(H$.$) herrickana? (Girty, 1909)$

Kirkbya sp. 1

Kirkbya sp. 2

Sargentina chatarameei Chitnarin, 2012

Geffenina posterodorsospina Chitnarin, 2012

Geisina sp.

Bairdia sp. K

Fabalicypris hathaithipae Chitnarin, n. sp.

Microcheilinella sp. G

Luizhinia naramasei Chitnarin, n. sp.

Acratia chongpani Chitnarin, n.sp.

Cryptobairdia sp.

Acratia mongkoli Chitnarin, n. sp.

Basslerella wipanuae Chitnarin, n. sp

Bairdia sp. J

?Pseudacanthoscapha sp.

Polytylites sp.

Cyathus caperata (Guan, 1978)

Cyathus elliptica Shi, 1987

Samarella viscusforma Chitnarin, 2012

Kempfina sp.

Bairdia cf. urodeloformis Chen, 1987

Bairdia deducta deducta Zalanyi, 1974

Polycope sp. A

Silenites sureeae Chitnarin, n. sp.

Bairdia piscariformis Chen, 1958

Polycope sp. C

Bairdia deweveri Crasquin, 2010

Liuzhinia praeantalayaensis Forel, 2010

Baschkirina ruchae Chitnarin, n. sp.

Baschkirina cf. ballei Crasquin, 2010

Bairdia lungtanensis Chen, 1958

Basslerella tota Chen \& Bao, 1986

Bairdia grotei Chitnarin, n. sp.

Bairdia trianguliformis Chen, 1958

Bairdia sp. E

Bairdiacypris sp. F

Bohlenatia sp.

Basslerella naresi Chitnarin, n. sp.

Polycope sp.B

Paraparchites chenshii Crasquin, 2010

Permoyoungiella sp.

Knightina sp.

Kirkbya sp.

Shemonaella sp. 1

Kloedenellidae indet.

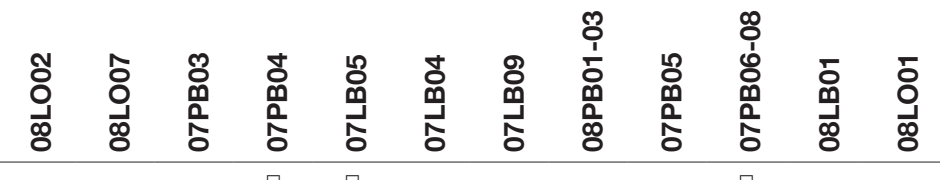

$\begin{array}{llllllllllll}- & - & - & \square & \square & - & - & - & - & \square & - & - \\ - & - & - & \square & - & - & - & - & - & - & - & -\end{array}$

$\begin{array}{lllllllllll}- & - & - & \square & - & - & - & - & - & - & - \\ - & - & - & \square & - & - & - & \square & - & - & -\end{array}$

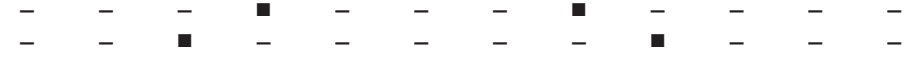

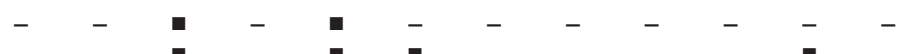

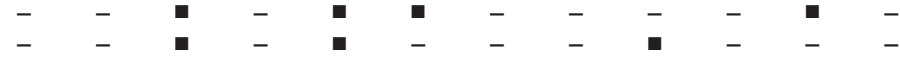

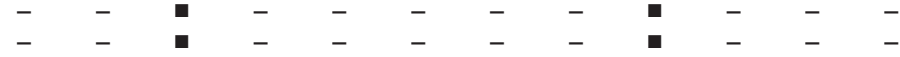

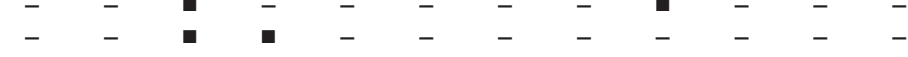

(2) 

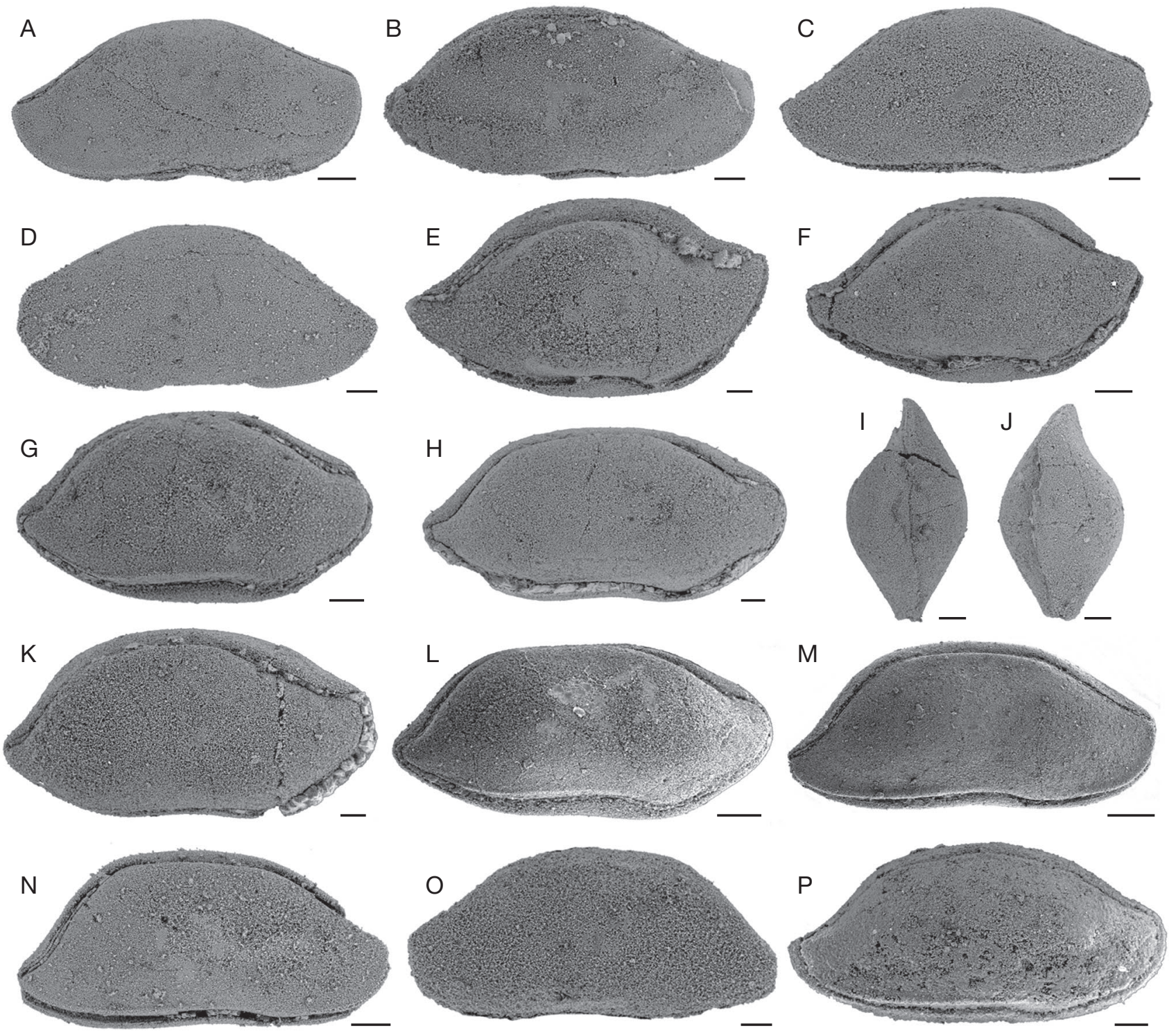

FIG. 3. - Ostracods from Indochina Block, Central Thailand. All specimens are stored at the Suranaree University of Technology Collections (Nakhon Ratchasima, Thailand), numbers SUT-09-xxxx. All the specimens are represented by complete carapaces. A-D, Bairdia khaokanaensis Chitnarin, n. sp.; A, holotype, right lateral view, SUT-09-2223, sample 07PB03-3; B, paratype, right lateral view, SUT-09-2226, sample 07PB03-3; C, paratype, right lateral view, SUT-092231, sample 07PB03-3; D, left lateral view, SUT-09-2232, sample 07PB03-5; E, F, I, J, Bairdia incisedorsa Chitnarin, n. sp.; E, holotype, right lateral view, SUT-09-2216, sample 07PB05-3; F, paratype, right lateral view, SUT-09-2215, sample 07PB05-3; I, paratype, dorsal view, SUT-09-2221, sample 07PB05-3; J, ventral view, paratype, SUT-09-2220, sample 07PB05-3. G, H, K, L Bairdia fontainei Chitnarin, n. sp.; G, holotype, right lateral view, SUT-09-2187, sample 07LB05-3; H, paratype, right lateral view, SUT-09-2182, sample 07PB04-5; K, paratype, right lateral view, SUT-09-2183, sample 07PB05-3; L, right lateral view, SUT-09-2178, sample 07LB05-5; M-P, Bairdia songthami Chitnarin, n. sp.; M, holotype, right lateral view, SUT-09-2283, sample 07LB05-5; N, paratype, right lateral view, SUT-09-2278, sample 07PB05-4; O, paratype, left lateral view, SUT-09-2281, sample 07PB03-1; P, right lateral view, SUT-09-2284, sample 07LB05-A2. Scale bars: $100 \mu \mathrm{m}$.

differentiated by its shorter and more convex $\mathrm{DB}$, the longer and more convex AVB and PVB. Bairdia khaokanaensis Chitnarin, n. sp. can also be compared to B. girtyi Sohn, 1960 from the Early Permian of South China (Chen \& Bao 1986: 115, pl. 2, figs 6, 7) in lateral outline but they can be differentiated by the longer and flat $\mathrm{DB}$ and the moderate overlapping at DB of the latter species. AVB and PVB of B. khaokanaensis Chitnarin, n. sp. are longer and more convex.
Bairdia incisedorsa Chitnarin, n. sp.

(Figs 3E-F, I-J)

TYPE MATERIAL. - Holotype: one complete carapace (SUT-09-2216; Fig. 3E); paratype, one complete carapace (SUT-09-2215; Fig. 3F).

ETYMOLOGY. — From diagnosis character.

MATERIAL EXAMINED. — Five complete and three incomplete carapaces. 
Dimensions. $-\mathrm{H}=0.46-0.50 \mathrm{~mm}, \mathrm{~L}=0.85-0.90 \mathrm{~mm}, \mathrm{H} / \mathrm{L}=$ 0.54-0.55, Holotype: $\mathrm{H}=0.501 \mathrm{~mm}, \mathrm{~L}=0.901 \mathrm{~mm}$; Paratype: $\mathrm{H}=0.469, \mathrm{~L}=0.855$.

TYPE HORIZON. - Sample 07PB05-3, Ban Naen Sawan I section, Tak Fa Formation, Roadian, Middle Permian.

Type locality. - Ban Naen Sawan I section (15² 54'11'N, $100^{\circ}$ 53'36”E), Phetchabun Province, Central Thailand.

OCCURRENCES. - Sample 07PB05-3, Ban Nean Sawan I section, Phetchabun Province, Tak Fa Formation, Middle Permian, Central Thailand.

DiAGNOSIS. - Species of Bairdia with robust carapace, strong overlap of $L V$ over $\mathrm{RV}$ in dorsal region, hinge invaginated, $\mathrm{AB}$ with very small radius of curvature and maximum of convexity located very high, tapering $\mathrm{PB}$ with maximum of curvature located at mid $\mathrm{H}$, slight overlap of LV on RV at AVB and PVB, dorsal view spindle-shaped.

\section{DESCRIPTION}

Carapace robust; dorsal outline broadly arched, DB of RV convex, DB of LV, hinge short and strongly invaginated; $\mathrm{ADB}$ short and concave; anterior end tapered and pointing upward, located above mid $\mathrm{H}$; AVB long and slightly convex, angle between $\mathrm{AVB}$ and $\mathrm{VB}$ is $120^{\circ}$; VB short, VB of RV concave, VB of LV slightly convex; PVB long and slightly convex, angle between $\mathrm{PVB}$ and $\mathrm{VB}$ is $155^{\circ}$; $\mathrm{PB}$ tapering and located at mid $\mathrm{H}$; PDB long and concave; anterior, posterior, anteroventral and posteroventral regions flattened laterally; maximum $\mathrm{H}$ located at mid L; surface smooth; LV larger and strongly overlaps RV at dorsal and ventral borders, slightly overlaps at AVB and PVB; in dorsal view, carapace spindle-shaped, with median part inflated and extremities strongly flattened.

\section{REMARKS}

Bairdia incisedorsa Chitnarin, n. sp. could be easily identifiable by its diagnostic characters. However, in lateral view, it is closed to B. permiana Hamilton, 1942 from the Guadalupian, Middle Permian of Texas (Hamilton 1942). The two species can be differentiated by dorsal characteristics and the lower $\mathrm{H} / \mathrm{L}$ ratio of $B$. incisedors Chitnarin, n. sp. The present species can be compared with B. subleguminoides Chen, 1987 from the Late Permian of South China (Shi \& Chen 1987) but the latter species is more elongated with longer anterior part and $\mathrm{DB}$ more convex at RV.

\section{Bairdia fontainei Chitnarin, n. sp.} (Figs 3G-H, K-L; 5)

Bairdia cf. piscariformis Chen in Chitnarin, Crasquin, Chonglakmani, Broutin, Grote \& Thanee et al. 2008: 347, 349, fig. 6 (1-2). n. syn.

TYPE MATERIAL. - Holotype, one complete carapace (SUT-092187; Fig. 3G); paratypes, two complete carapaces (SUT-09-2182; Fig. 3H and SUT-09-2178; Fig. 3L).

Eтymology. - In honour of Dr Henry Fontaine, France.

MATERIAL EXAMINED. - Ten complete carapaces.

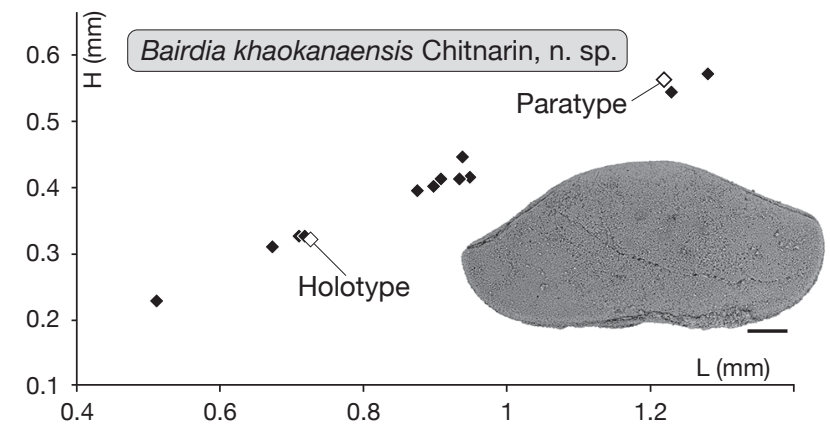

FIG. 4. - Height (H)-length (L) diagram of Bairdia khaokanaensis Chitnarin, n. sp. Scale bar: $100 \mu \mathrm{m}$.

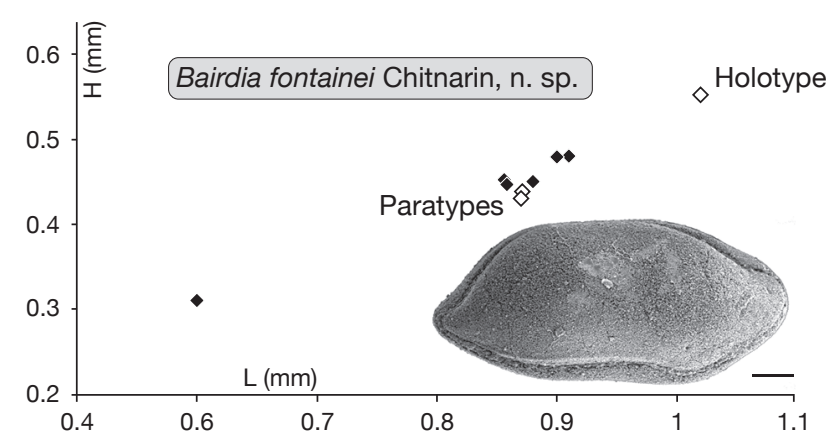

FIG. 5. - Height (H)-length (L) diagram of Bairdia fontainei Chitnarin, n. sp. Scale bar: $100 \mu \mathrm{m}$.

Dimensions. $-\mathrm{H}=0.44-0.48 \mathrm{~mm}, \mathrm{~L}=0.87-0.92 \mathrm{~mm}, \mathrm{H} / \mathrm{L}=0.48$ 0.53 , Holotype: $\mathrm{H}=0.55 \mathrm{~mm}, \mathrm{~L}=1.02 \mathrm{~mm}$; Paratype (Fig. $3 \mathrm{H}$ ): $\mathrm{H}=0.43, \mathrm{~L}=0.87$; Paratype (Fig. $3 \mathrm{~L}$ ): $\mathrm{H}=0.44, \mathrm{~L}=0.91$ (Fig. 5).

TYPE HORIZON. - Sample number 07LB05-5, Ta Kli section, Tak Fa Formation, Artinskian, Early Permian.

Type LOCAlity. - Ta Kli section (15¹9’05”N, 100²2’46”E), Nakhon Sawan Province, Central Thailand.

Occurrences. - Sample 02TH46-07, Bung Sam Phan section, Tak Fa Formation, (Chitnarin et al. 2008), Phetchabun Province, Middle Permian, Central Thailand; sample 07LB05-05, Ta Kli section, Tak Fa Formation, Nakhon Sawan Province, Middle Permian, Central Thailand; sample 07PB05-3, Ban Nean Sawan I section, Phetchabun Province, Tak Fa Formation, Middle Permian, Central Thailand; samples 07PB04-2 and 07PB04-5, Nong Phai section, Pha Nok Khao Formation, Phetchabun Province, Early Permian, Central Thailand.

DiAGNOSIS. - Species of Bairdia with spindle-shape carapace and a ventral ridge along $\mathrm{VB}$ of $\mathrm{RV}, \mathrm{DB}$ and $\mathrm{VB}$ nearly straight and of equal length, $\mathrm{H}$ maximum at mid- $\mathrm{L}$, distinct overlap of $\mathrm{LV}$ on $\mathrm{RV}$ at $\mathrm{ADB}$ and $\mathrm{PDB}$.

\section{DESCRIPTION}

Spindle shape carapace in lateral view; DB and VB nearly straight and of equal length; at RV, $\mathrm{ADB}$ straight and has an angle of $150^{\circ}$ with $\mathrm{DB}, \mathrm{ADB}$ of $\mathrm{LV}$ slightly convex; $\mathrm{AB}$ rounded with medium radius of curvature, maximum convexity located at mid $\mathrm{H} ; \mathrm{AVB}$ of both valves straight, angle between $\mathrm{AVB}$ and $\mathrm{VB}$ is $150^{\circ}$; VB of RV slightly concave, VB of LV slightly concave to nearly straight; $\mathrm{PVB}$ of both valves straight, angle between PVB and VB is $150^{\circ}$; PB tapering, 


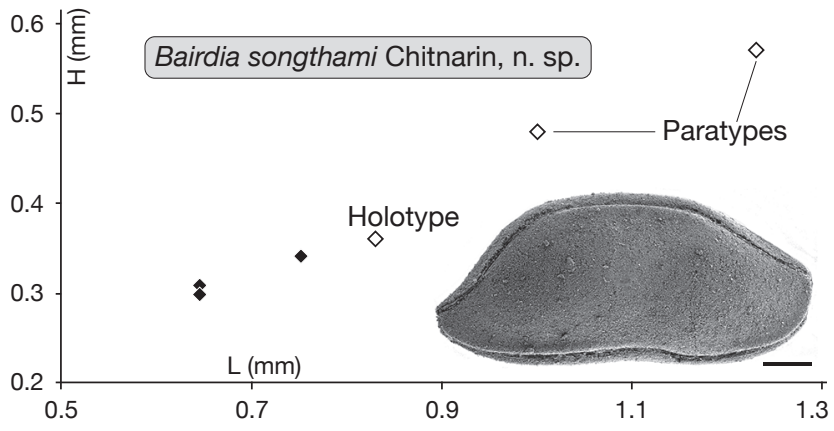

FIG. 6. - Height (H)-length (L) diagram of Bairdia songthami Chitnarin, n. sp. Scale bar: $100 \mu \mathrm{m}$.

maximum convexity located at mid $\mathrm{H}$; PDB of both valves straight, angle between $\mathrm{PDB}$ and $\mathrm{DB}$ is about $135^{\circ}$; surface smooth; presence of a ventral ridge along $\mathrm{VB}$ of $\mathrm{RV}$; greatest $\mathrm{H}$ in the median region; $\mathrm{LV}$ strongly overlaps $\mathrm{RV}$ at $\mathrm{ADB}$, PDB and VB, slightly overlaps at both ends.

\section{REMARKS}

Bairdia fontainei Chitnarin, n. sp. includes the species identified as B. cf. piscariformis Chen, 1958 from the Middle Permian limestone in Phetchabun area (Chitnarin et al. 2008). However, it can be differentiated from $B$. piscariformis Chen, 1958 from the Early Permian of South China (Chen 1958: 245, pl. 4, figs 9-10) by the nearly straight DB and VB, the equal length of both valves, the straight AVB and PVB and the presence of ventral ridge on RV. B. fontainei Chitnarin, n. sp. is close to B. guadalupiana Hamilton, 1942 from the Middle Permian of Texas (Hamilton 1942) in general outline but the anterior part of the latter species is more rounded and with larger radius of curvature.

\section{Bairdia songthami Chitnarin, n. sp.} (Figs 3M-P; 6)

TYPE MATERIAL. - Holotype: one complete carapace (SUT-092283, Fig. 3M); paratypes: two complete carapaces (SUT-09-2278, Fig. 3N and SUT-09-2284; Fig. 3P).

ETYMOLOGY. - In honour of Dr Wichanet Songtham, Northeastern Institute of Petrified Wood and Mineral Resources, Nakhon Ratchasima Rajabhat University, Thailand.

MATERIAl EXAMined. - Six complete carapaces.

Dimensions. $-\mathrm{H}=0.30-0.57 \mathrm{~mm}, \mathrm{~L}=0.64-1.12 \mathrm{~mm}, \mathrm{H} / \mathrm{L}=$ 0.45-0.46, Holotype: $\mathrm{H}=0.360 \mathrm{~mm}, \mathrm{~L}=0.83 \mathrm{~mm}$; Paratype (Fig. $3 \mathrm{~N}$ ): $\mathrm{H}=0.47, \mathrm{~L}=1.00$; Paratype (Fig. $3 \mathrm{P}$ ): $\mathrm{H}=0.57 \mathrm{~mm}$, $\mathrm{L}=1.23 \mathrm{~mm}$ (Fig. 6).

Type HORIZON. - Sample 07LB05-2, Ta Kli section, Tak Fa Formation, Middle Permian.

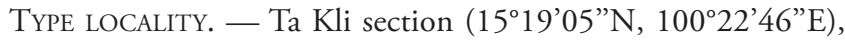
Nakhon Sawan Province, central Thailand.

OCCURRENCES. - Sample 07PB03-1, Khao Kana section, Pha Nok Khao Formation, Phetchabun Province, Early Permian; sample 07PB05-6, Ban Naen Sawan I section, Tak Fa Formation, Phetchabun
Province, Middle Permian; samples 07LB05-2 and 07LB05-A2, Ta Kli section, Tak Fa Formation, Nakhon Sawan Province, Middle Permian, Central Thailand.

Diagnosis. - Species of Bairdia with flat carapace, DB nearly straight and long, AVB, VB, PVB flattened laterally and underlined by a ridge all along ventral part at RV.

\section{DESCRIPTION}

Carapace flat and subtrapezoid; dorsal outline broadly arched; DB of both valves long, straight, horizontal to slightly inclined to anterior; $\mathrm{ADB}$ long, angle between $\mathrm{ADB}$ and $\mathrm{DB}$ is $150^{\circ}$; angle between $\mathrm{PDB}$ and $\mathrm{DB}$ is $135^{\circ}$; $\mathrm{AB}$ rounded with medium radius of curvature, maximum convexity located below mid $\mathrm{H}$; AVB convex; VB concave; PVB long and slightly convex; AVB, $\mathrm{VB}, \mathrm{PVB}$ flattened and underlined by a fine ridge all along the ventral margin at RV; PB tapered, located at lower fourth of $\mathrm{H}$; maximum of $\mathrm{H}$ located at mid L; surface smooth; LV larger than $\mathrm{RV}$, and moderately overlaps RV all around the carapace, except at anterior and posterior ends; in dorsal view, carapace very thin.

\section{REMARKS}

Bairdia songthami Chitnarin, n. sp. can be compared to B. girtyi from the Early Permian of South China (Chen 1958); however, in the present Thai material, the maximum of convexity of $A B$ and $\mathrm{PB}$ is located lower and DB is longer. Bairdia songthami Chitnarin, n. sp. differs from B. fontainei Chitnarin, n. sp. by its more convex AVB and PVB; here, AVB, VB and PVB are flattened latteraly and the ventral margin is underlined by a ventral ridge.

Bairdia grotei Chitnarin, n. sp. (Figs 7A-F; 8)

Bairdia sp. 1 - Crasquin et al. 2010b: 25, pl. 1, fig. 23. n. syn.

TYPE MATERIAL. - Holotype: one complete carapace (SUT-092206, Fig. 7F); paratypes: two complete carapaces (SUT-09-2189, Fig. 7B and SUT-09-2195, Fig. 7E).

EтYMOLogy. - In honour of Dr Paul J. Grote, Suranaree University of Technology, Thailand.

MATERIAL EXAMINED. - 11 complete carapaces.

DimeNSIONS. $-\mathrm{H}=0.35-0.45 \mathrm{~mm}, \mathrm{~L}=0.62-0.74 \mathrm{~mm}, \mathrm{H} / \mathrm{L}=0.57-$ 0.61 , Holotype: $\mathrm{H}=0.636 \mathrm{~mm}, \mathrm{~L}=1.07 \mathrm{~mm}$; Paratype (Fig. $7 \mathrm{~B}$ ): $\mathrm{H}=0.45 \mathrm{~mm}, \mathrm{~L}=0.706$; Paratype (Fig. $7 \mathrm{E}): \mathrm{H}=0.45 \mathrm{~mm}, \mathrm{~L}=$ $0.74 \mathrm{~mm}$ (Fig. 8).

Type Horizon. - Sample 07PB04-2, Nong Phai section, Pha Nok Khao Formation, Asselian-Sakmarian, Early Permian.

TyPe LOCALITY. - Nong Phai section (1601'06”N, 10058'59”E), Phetchabun Province, central Thailand.

OCCURRENCES. - Jadar Block, NW Serbia, latest Permian. Samples 07PB04-2 and 07PB04-5, Nong Phai section, Pha Nok Khao Formation, Phetchabun Province, Early Permian, Central Thailand; samples 07LB05-A1, 07LB05-B2, 07LB05-D2, Ta Kli section, Nakhon Sawan Province, Middle Permian, Central Thailand; sample 07LB04-17, Phu Lam Yai section, Tak Fa Formation, Nakhon Sawan Province, Early Permian?, Central Thailand; sample 07LB09-1, Khao Phu Chongkho 

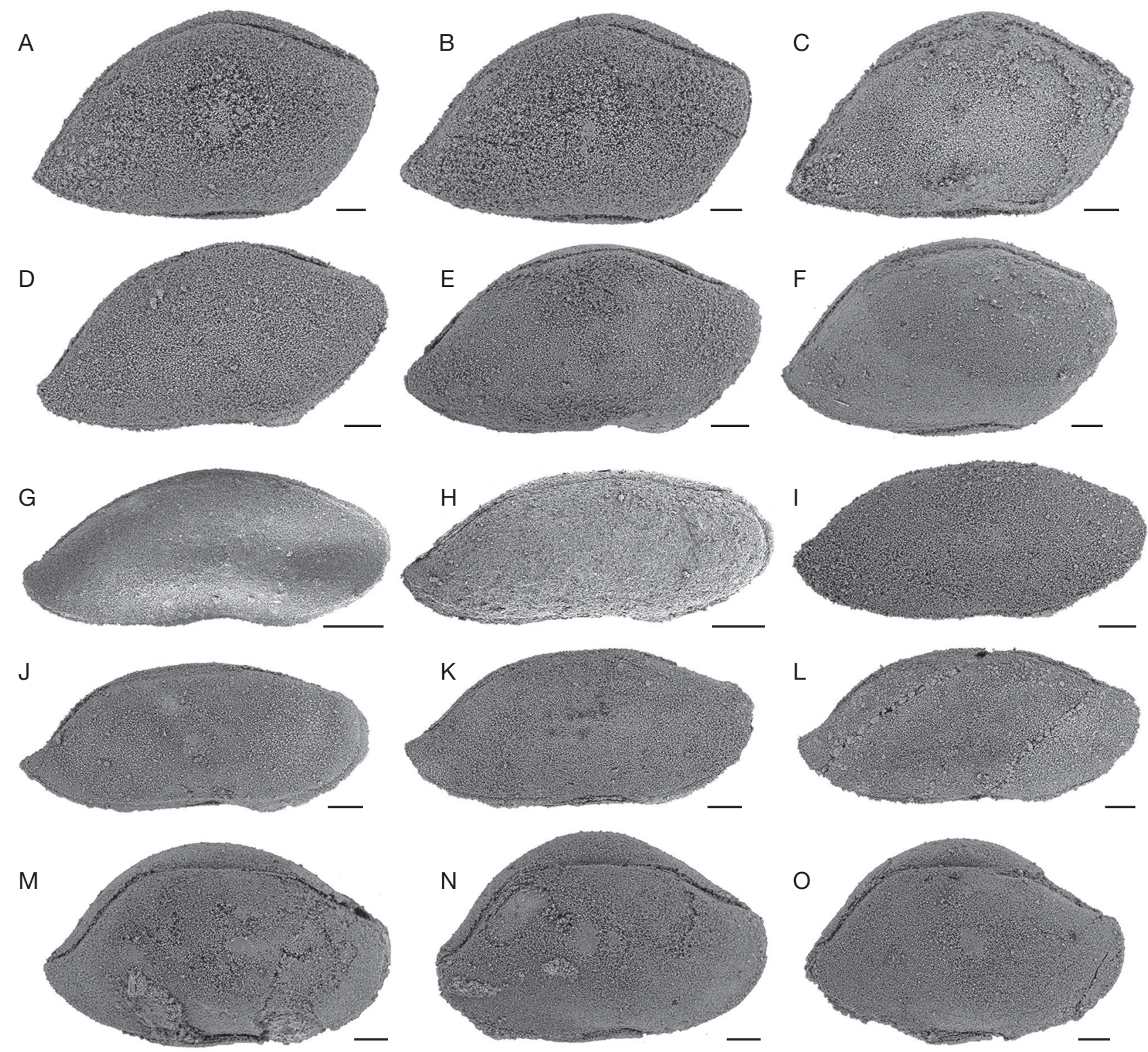

FIG. 7. - Ostracods from Indochina Block, Central Thailand. All specimens are stored at the Suranaree University of Technology Collections (Nakhon Ratchasima, Thailand) numbers SUT-09-xxxx. All the specimens are complete carapaces in right lateral view. A-F, Bairdia grotei Chitnarin, n. sp.; A, SUT-09-2188, sample 07PB04-2; B, paratype, SUT-09-2189, sample 07PB04-2; C, SUT-09-2192, sample 07LB05-B2; D, SUT-09-2203, sample 07PB04-2; E, paratype, SUT-09-2204, sample 08LO07-1; F, holotype, SUT-09-2206, sample 08LO02-11. G-L, Bairdia cf. fangnianqiaoi Crasquin, 2010; G, SUT-09-2260, sample 07LB05-4; H, SUT09-2263, sample 08LB01-1; I, SUT-09-2261, sample 07LB05-B3; J, SUT-09-2270, sample 07LB05-B3; K, SUT-09-2271, sample 07PB04-2; L, SUT-09-2279, sample 07PB04-2. M-O, Bairdia cf. B.? mianyangensis Chen, 1982; M, SUT-09-2254, sample 07PB03-3; N, SUT-09-2252, sample 07PB03-3; O, SUT-09-2250, sample 07PB03-3. Scale bars: $100 \mu \mathrm{m}$. .

section, Tak Fa Formation, Nakhon Sawan Province, Middle Permian, Central Thailand; sample 08LO02-11, Tham Nam Maholan section, Nam Maholan Formation, Loei Province, Early Permian, Northeastern Thailand; 08LO07-1, Sak Chai Quarry section, Nam Maholan Formation, Loei Province, Early Permian, Northeastern Thailand.

Diagnosis. - Species of Bairdia whith spindle shape, PB located at lower $30 \%$ of $\mathrm{H}, \mathrm{AB}$ located at $75 \%$ of $\mathrm{H}, \mathrm{PDB}$ long and parallel to $\mathrm{AVB}, \mathrm{H} / \mathrm{L}=0.57-0.61$.

\section{DESCRIPTION}

Carapace spindle-shaped; dorsal outline broadly arched, maximum of convexity of $\mathrm{AB}$ at upper $30 \%$ of $\mathrm{H}$, of $\mathrm{PB}$ below lower fourth of $\mathrm{H}$; DB straight and slightly inclined backward $\left(10^{\circ}\right)$; ADB straight, angle with $\mathrm{DB}$ of $130^{\circ}$; $A B$ rounded with medium radius of curvature, maximum convexity located above mid $\mathrm{H}$; AVB long and nearly straight, angle between AVB and VB is $140^{\circ}$; VB straight at LV, slightly concave at RV; PVB long, slightly convex; $\mathrm{PB}$ rounded with small radius of curvature, maximum convexity located below lower fourth of $\mathrm{H}$; PDB long and straight, parallel to AVB; angle between PDB and DB of $150^{\circ}$; LV slightly overlaps RV all around the carapace, overlap moderate at $\mathrm{DB}$ and $\mathrm{ADB}$. 


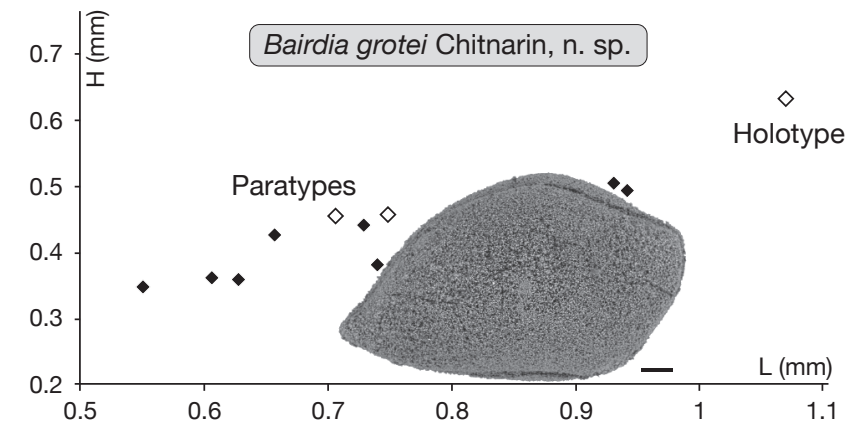

FIG. 8. - Height (H)-length (L) diagram of Bairdia grotei Chitnarin, n. sp. Scale bar: $100 \mu \mathrm{m}$.

\section{REMARKS}

Bairdia grotei Chitnarin, n. sp. can be compared to B. gaelleae Crasquin, 2010 from the latest Permian of Meishan, South China (Crasquin et al. 2010a) in lateral view; however here the $\mathrm{AB}$ has a smaller radius of curvature and $\mathrm{ADB}$ and $\mathrm{DB}$ are straighter.

\section{Bairdia cf. fangnianqiaoi Crasquin, 2010} (Fig. 7G-L)

Bairdia cf. fangnianqiaoi Crasquin in Crasquin et al., 2010a: 342, 344, fig. 9K-N.

MATERIAL EXAMINED. - 13 complete carapaces.

OCCURrenCES. - Sample 07PB04-2, Nong Phai section, Pha Nok Khao Formation, Phetchabun province, Early Permian, Central Thailand; samples 07LB05-3, 07LB05-4, 07LB05-B3, 07LB05-C1, Ta Kli section, Tak Fa Formation, Nakhon Sawan Province, Middle Permian, Central Thailand; sample 08LB01-1, Khao Som Phot section, Tak Fa Formation, Lopburi Province, Middle Permian, Central Thailand.

Dimensions. $-\mathrm{H}=0.20-0.60 \mathrm{~mm}, \mathrm{~L}=0.60-1.07 \mathrm{~mm}, \mathrm{H} / \mathrm{L}=$ $0.36-0.43$

\section{REMARKS}

The specimens recovered are very similar to Bairdia fangnianqiaoi Crasquin, 2010 from the latest Permian of Meishan section (Crasquin et al. 2010a) in lateral view but can be differentiated by a smaller $\mathrm{H} / \mathrm{L}$ ratio and regularly arched dorsal outline at LV.

\section{Bairdia cf. mianyangensis Chen, 1982}

(Fig. 7M-O)

Bairdia cf. mianyangensis Chen, 1982: 123, pl. IV, figs 14-18.

MAterial EXAMined. - Four complete and three incomplete carapaces.

OCCURRENCES. - Samples 07PB03-1 and 07PB03-3 Khao Kana section, Pha Nok Khao Formation, Phetchabun Province, Early Permian.

Dimensions. $-\mathrm{H}=0.47-0.64 \mathrm{~mm}, \mathrm{~L}=0.61-1.11 \mathrm{~mm}, \mathrm{H} / \mathrm{L}=$ $0.57-0.58$.
REMARKS

Chen (in Chen \& Shi 1982: 123, pl. 4, figs 14-18) described a new species Bairdia? mianyangensis from the latest Permian of Mianyang, Hubei, South China. Their specimens are incomplete carapaces but such characters as unusual DB of RV inclined frontward with strong overlap at DB and flattened AVB and PVB can be distinguished. The specimens recovered here resemble B.? mianyangensis in lateral outline and the mentioned characters; however, our specimens are longer and the $\mathrm{VB}$ is less concave than those of $B$.? mianyangensis.

\section{Bairdia guangxiensis Guan, 1978}

(Fig. 9A-C)

Bairdia guangxiensis Guan in Guan et al., 1978: 154, pl. 38, figs 3-4. Chen \& Shi 1982: 122, pl. 5, fig. 17. — Chen \& Bao 1986: 114, pl.3, figs 7-8. — Shi \& Chen 1987: 31, pl. 3, figs 1-9; 2002: 67, pl. 5, figs 1-9, pl. 28, figs 1-2.

MATERIAL EXAMINED. - Two complete and 14 incomplete carapaces.

Occurrences. - Wugang, Hunan Province, Early Permian (Guan et al. 1978); Nantong section, (Chen \& Shi 1982); WellBao-1, Chihsia Limestone, Jiangsu Province, Early Permian (Chen \& Bao 1986); Meishan section, Changxing, Zhejiang, South China, latest Permian (Shi \& Chen 1987); Matan and Pingding sections, Guangxi, South China, Late Permian (Shi \& Chen 2002); samples 07PB06-3, 07PB07-4 and 07PB08-2, Ban Naen Sawan II section, Tak Fa Formation, Phetchabun Province, Middle Permian, Central Thailand.

Dimensions. $-\mathrm{H}=0.55-0.84 \mathrm{~mm}, \mathrm{~L}=0.82-1.18 \mathrm{~mm}, \mathrm{H} / \mathrm{L}=$ $0.66-0.71$

\section{REMARKS}

Bairdia guangxiensis can be easily recognized by presence of ventral beak-like lateral inflations on both valves and flat ventral area. Small differences can be recognized among our specimens; for example, DB of LV is convex unlike the flat DB of those described from Late Permian of Guangxi (Shi \& Chen 2002: pl. 5, figs 1 and 9), and are of medium size.

\section{Bairdia cf. bassoni Crasquin, 2010} (Fig. 9E-F)

Bairdia cf. bassoni Crasquin, 2010: 340, fig. 7Y, Z, C'-F'.

Material EXAMined. - Eight complete carapaces.

OCCURrEnCES. - Sample 07PB03-3, Khao Kana section, Pha Nok Khao Formation, Phetchabun province, Early Permian, Central Thailand.

Dimensions. $-\mathrm{H}=0.364-0.529 \mathrm{~mm}, \mathrm{~L}=0.617-0.930 \mathrm{~mm}$, $\mathrm{H} / \mathrm{L}=0.56-0.58$.

\section{REMARKS}

The specimens attributed to Bairdia cf. bassoni Crasquin, 2010 are characterized by regularly arched DB, maximal $\mathrm{H}$ located at mid L, and both ends compressed laterally; however, $\mathrm{AB}$ and $\mathrm{PB}$ are smaller than those of B. bassoni Crasquin, 2010 

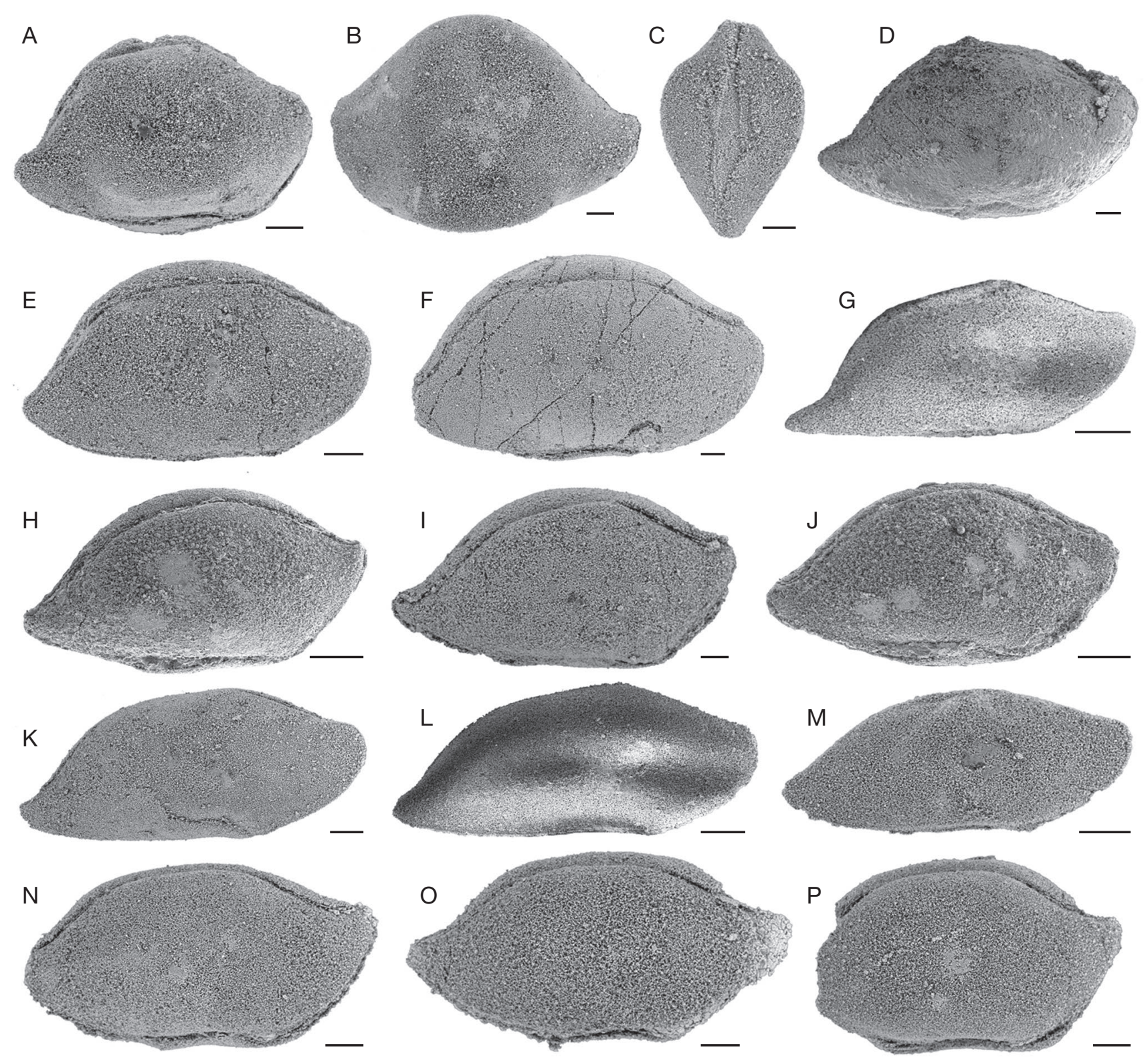

FIG. 9. - Ostracods from Indochina Block, Central Thailand. All specimens are stored at the Suranaree University of Technology Collections (Nakhon Ratchasima, Thailand) numbers SUT-09-xxxx. All the specimens are represented by complete carapaces. A-C, Bairdia guangxiensis Guan, 1978; A, right lateral view, SUT-09-2207, sample 07PB07-4; B, left lateral view, SUT-09-2208, sample 07PB06-3; C, ventral view, SUT-09-2213, sample 07PB07-3. D, Bairdia cf. zhongyingensis Wang, 1978 sensu Chen \& Bao, 1986; right lateral view, SUT-09-2339, sample 07LB05-A2; E, F, Bairdia bassoni Crasquin, 2010; E, right lateral view, SUT-09-2117, sample 07PB03-3; F, right lateral view, SUT-09-2118, sample 07PB03-3; G-J, Bairdia urodeloformis Chen, 1987; G, right lateral view, SUT-09-2332, sample 08LB01-1; H, right lateral view, SUT-09-2325, sample 07LB05-5; I, right lateral view, SUT-09-2335, sample 08PB05-3; J, right lateral view, SUT-09-2338, sample 07LB05-5; K-M, Bairdia cf. urodeloformis Chen, 1987; K, right lateral view, SUT-09-2151, sample 07LB05-B2; L, right lateral view, SUT-09-2152, sample 07LB05-3; M, right lateral view, SUT-09-2156, sample 08LO02-2; N-P, Bairdia sp. A; N, right lateral view, SUT-09-2285, sample 07PB04-2; O, right lateral view, SUT-09-2291, sample 07PB04-2; P, right lateral view, SUT-09-2290, sample 07PB04-2. Scale bars: $100 \mu \mathrm{m}$.

(Crasquin et al. 2010a) from the latest Permian of Meishan section, Eastern China. The overlap at DB in our specimens is stronger.

\section{Bairdia urodeloformis Chen, 1987}

(Fig. 9G-J)

Bairdia urodeloformis Chen in Shi \& Chen, 1987: 40, pl. 4, figs 1723. - Crasquin et al. 2010a: 348, figs 7G-N.
Rectobairdia firmata Chen in Chen \& Shi, 1982: pl. 7, fig. 10 only.

Bairdia macdonelli Harlton, 1929b: 157, pl. 3, figs 7a, b. — Shi \& Chen 1987: 35, pl. 1, figs 1-7, pl. 18, figs 1-4; 2002: 63, pl. 2, figs 1-4.

Material eXamined. - One incomplete and eight complete carapaces.

OCCURRENCES. - Meishan section, Zhejiang Province, latest Permian (Shi \& Chen 1987; Crasquin et al. 2010a); Matan and Pingding sections, Guangxi, South China, Late Permian (Shi \& Chen 2002); sample 07PB04-2, Nong Phai section, Pha Nok Khao 
Formation, Phetchabun Province, central Thailand, Early Permian; samples 07LB05, 07LB05-A2, Ta Kli section, Tak Fa Formation, Nakhon Sawan province, Middle Permian, central Thailand; sample 07PB05-3, Ban Naen Sawan I section, Tak Fa Formation, Phetchabun Province, central Thailand, Middle Permian; .

DiMENSIONS. $-\mathrm{H}=0.25-0.51 \mathrm{~mm}, \mathrm{~L}=0.54-1.25 \mathrm{~mm}, \mathrm{H} / \mathrm{L}=0.41-0.46$

\section{REMARKS}

Such characters as long carapace, broadly arched dorsal outline of LV, angulated dorsal outline of RV with small AB located very high and small $\mathrm{PB}$ located very low induce the attribution to Bairdia urodeloformis Chen, 1987 described from the latest Permian of South China (Shi \& Chen 1987).

\section{Bairdia cf. urodeloformis Chen, 1987} (Fig. 9K-M)

Bairdia urodeloformis Chen in Shi \& Chen, 1987: 40, pl. 4, figs 17- 23. Material EXAMINED. - 12 complete carapaces.

OCCURRENCES. - Sample 08LO02-2, Tham Nam Maholan section, Nam Maholan Formation, Loei Province, northeastern Thailand, Early Permian; samples 07LB05-A1, 07LB05-B2 and 07LB05-B3, Ta Kli section, Tak Fa Formation, Nakhon Sawan Province, Central Thailand, Middle Permian; sample 08LB01-1, Khao Som Phot section, Tak Fa Formation, Lopburi Province, central Thailand, Middle Permian.

Dimensions. $-\mathrm{H}=0.22-0.45 \mathrm{~mm}, \mathrm{~L}=0.63-1.05 \mathrm{~mm}, \mathrm{H} / \mathrm{L}=$ $0.34-0.43$.

\section{REMARKS}

The specimens are related to Bairdia urodeloformis from the latest Permian of South China (see references above) by their long carapace, small $\mathrm{AB}$ with maximum of convexity located very high and small $\mathrm{PB}$ with maximum of convexity located very low. The differences are the longer carapace, the more convex PVB, and the overlap along dorsal border.

\section{Bairdia cf. calida Chen, 1958}

(Fig. 10A, B)

Bairdia calida Chen, 1958: 243, pl. IV, figs 9-15.

MATERIAL EXAMINED. - One complete and two incomplete carapaces.

OCCURRENCES. - Sample 07PB03-7, Khao Kana section, Pha Nok Khao Formation, Phetchabun province, Central Thailand, Early Permian; sample 07PB05-3, Ban Naen Sawan I section, Pha Nok Khao Formation, Phetchabun province, Central Thailand, Middle Permian.

Dimensions. $-\mathrm{H}=0.715-0.747 \mathrm{~mm}, \mathrm{~L}=1.22-1.33 \mathrm{~mm}, \mathrm{H} / \mathrm{L}=$ $0.56-0.58$.

\section{REMARKS}

The specimens are close to Bairdia calida Chen, 1958 from the Early Permian of Jiangsu Province, Eastern China (Chen 1958) which is characterized by a narrow ventral ridge on $\mathrm{RV}$ and the dorsal ridge on LV. In our specimens, the ventral ridge on RV is observed; though, the dorsal ridge on $\mathrm{LV}$ is not recognized.

\section{Bairdia cf. zhongyingensis Wang, 1978}

(Fig. 9D)

Bairdia zhongyingensis Wang, 1978: 16, pl. III, fig. 2a-c.

MATERIAL EXAMINED. - One complete carapace.

OCCURRENCES. - Sample 07LB05-A2, Ta Kli section, Tak Fa Formation, Nakhon Sawan Province, central Thailand, Middle Permian.

Dimensions. $-\mathrm{H}=0.712 \mathrm{~mm}, \mathrm{~L}=1.31 \mathrm{~mm}, \mathrm{H} / \mathrm{L}=0.54$.

\section{REMARKS}

The specimen resembles Bairdia zhongyingensis Wang, 1978 from the Late Permian of South China (Wang 1978) in lateral outline, especially for the small radius of curvature of $A B$, small radius of curvature of $\mathrm{PB}$ and concave $\mathrm{VB}$; however, radius of curvature of $A B$ of our specimen is largerand higher and the overlap of LV on RV is not clear.

\section{Bairdia beedei Ulrich \& Bassler, 1906}

(Fig. 10C, D)

Bairdia beedei Ulrich \& Bassler, 1906: 161, pl. XI, figs 19, 20. Chen 1958: 249, pl. 5, figs 1-4.

Material eXAmined. - Five complete carapaces.

OCCURRENCES. - Kwanshan and Lungtan sections, Jiangsu Province, Early Permian (Chen 1958); samples 07PB03-1, 07PB03-7, Khao Kana section, Pha Nok Khao Formation, Phetchabun Province, Early Permian; sample 07PB04-2, Nong Phai section, Pha Nok Khao Formation, Phetchabun Province, Central Thailand, Early Permian.

Dimensions. $-\mathrm{H}=0.49-0.86 \mathrm{~mm}, \mathrm{~L}=0.82-1.13 \mathrm{~mm}, \mathrm{H} / \mathrm{L}=$ $0.54-0.57$

\section{REMARKS}

Our specimens have elongate, subfusiform carapace in lateral view with strong overlap of $L V$ on $R V$ at $D B$. DB of RV is steeply arched, DB of LV is straight and the VB is broadly convex. The carapaces of the studied specimens are longer than Bairdia beedei Ulrich \& Bassler from Late Carboniferous of North America (Ulrich \& Bassler 1906).

\section{Bairdia altiarcus Chen, 1958}

(Fig. 10E, F)

Bairdia altiarcus Chen, 1958: 223, pl. 3, figs 21-23, pl. 4, figs 11, 12. - Crasquin et al. 2008a: pl. 2, figs 5-6. - Yuan et al. 2009: pl. 1, fig. 17.

Non Cryptobairdia altiarca - Kozur 1985a: 231, table II, pl. 3, fig. 1. Cryptobairdia altiarcus - Shi \& Chen 2002: 69, pl. 7, figs 1-12.

Material eXAmined. - One incomplete and eleven complete carapaces. 

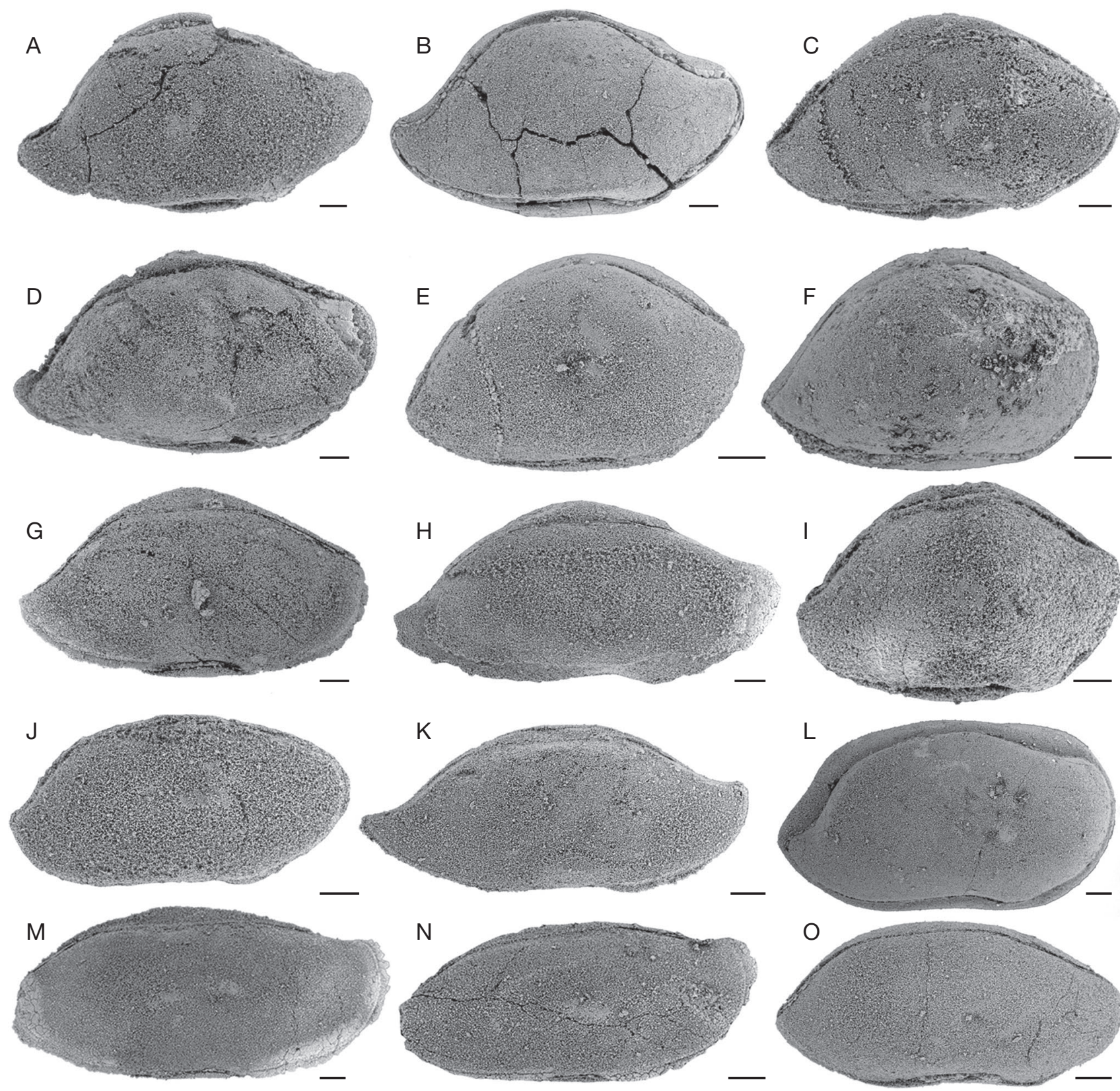

FIG. 10. - Ostracods from Indochina Block, Central Thailand. All specimens are stored at the Suranaree University of Technology Collections (Nakhon Ratchasima, Thailand) numbers SUT-09-xxxx. All the specimens are complete carapaces in right lateral view. A, B, Bairdia cf. calida Chen, 1958; A, SUT09-2127, sample 07PB03-7; B, SUT-09-2128, sample 07PB25-3; C, D, Bairdia beedei Ulrich \& Bassler, 1906; C, SUT-09-2685, sample 07PB03-3; D, SUT09-2687, sample 07PB04-2; E, F, Bairdia altiarcus Chen, 1958; E, SUT-09-2105, sample 07LB05-D2; F, SUT-09-2101, sample 07LB05-D3; G, H, Bairdia trianguliformis Chen, 1958; G, SUT-09-2318, sample 08LO07-10; H, SUT-09-2319, sample 08LO07-1; I, Kempfina cf. qinglaii (Crasquin, 2008), SUT-09-2533, sample 07PB03-7; J, Bairdia sp. D SUT-09-2300, sample 07LB09-2; K, Bairdia sp. E, SUT-09-2301, sample 08LO02-10; L, Bairdia sp. F, SUT-09-2311, sample 07LB05-5; M, N, Bairdia sp. G; M, SUT-09-2307, sample 07PB04-2; N, SUT-09-2306, sample 07PB04-2; 0, Bairdia sp. H, SUT-09-2310, sample 07PB05-6. Scale bars: $100 \mu \mathrm{m}$.

OCCURRENCES. - Kwanshan and Lungtan sections, Chihsia Limestone, Nanking Province, Early Permian (Chen 1958); Matan and Pingding sections, Guangxi, South China, Late Permian (Shi \& Chen 2002); Lercara Formation, Sicily, Italy, Middle Triassic (Crasquin et al. 2008a); samples 07LB05-B1, 07LB05-C3, 07LB05-D2 and 07LB05-D3, Ta Kli section, Tak Fa Formation, Nakhon Sawan Province, Central Thailand, Middle Permian.

Dimensions. $-\mathrm{H}=0.47-0.896 \mathrm{~mm}, \mathrm{~L}=0.74-1.30 \mathrm{~mm}, \mathrm{H} / \mathrm{L}=$ $0.61-0.68$.

\section{REMARKS}

The specimens recovered in this study can be easily assigned to Bairdia altiarcus from the Early Permian of Eastern China (Chen 1958) by their lateral characters such as highly arched dorsal outline, $\mathrm{AB}$ with large radius of curvature, acuminate and slightly upturned PB. However, some carapaces are compressed and distorted. Generally, our specimens are smaller than the Chinese specimens. 


\section{Bairdia trianguliformis Chen, 1958}

(Fig. 10G-H)

Bairdia trianguliformis Chen, 1958: 244, pl. 6, figs 9-12. - Chen \& Shi 1982: 121, pl. 4, figs 6-8. — Shi \& Chen 2002: 66, pl. 4, figs 3-9.

MATERIAl EXAMINED. - Two complete and three incomplete carapaces.

OCCurrences. - Kwanshan and Lungtan sections, Jiangsu Province, Early Permian (Chen 1958); Nantong section, Jiangsu Province, latest Permian (Chen \& Shi 1982); Matan and Pingding sections, Guangxi province, Late Permian (Shi \& Chen 2002); sample 08LO02-9, Tham Nam Maholan section, Nam Maholan Formation, Loei province, Northeastern Thailand, Early Permian; samples 08LO07-1 and 08LO07-10, Sak Chai Quarry section, Pha Nok Khao Formation, Loei Province, Northeastern Thailand, Early Permian; samples 07LB05-B1 and 07LB05-C3, Ta Kli section, Tak Fa Formation, Nakhon Sawan province, Central Thailand, Early Permian.

Dimensions. $-\mathrm{H}=0.61-0.89 \mathrm{~mm}, \mathrm{~L}=1.19-1.79 \mathrm{~mm}, \mathrm{H} / \mathrm{L}=$ $0.48-0.50$.

\section{REMARKS}

Bairdia trianguliformis from the Early Permian of Eastern China (Chen 1958) can be recognized by the strong overlap of $\mathrm{LV}$ on $\mathrm{RV}$ in dorsal region that makes a triangular area in the central of DB. The carapace is long and thin. Posterior end is tapering.

Bairdia deweveri Crasquin, 2010

(Fig. 12A-C)

Bairdia deweveri Crasquin in Crasquin, Forel, Feng, Yuan, Baudin \& Collin et al. 2010a: 342, figs 7O-T.

Bairdia cf. trianguliformis Chen in Shi \& Chen, 1987: 37, pl. 2, figs 1-8.

Bairdia galei Croneis \& Thurman, 1939: 325, pl. 7, fig. 21. Shi \& Chen 1987: 37, pl. 1, figs 19-22. — Crasquin et al. 2008b: pl. 2, figs 11,12 .

MATERIAL EXAMINED. - Ten complete carapaces.

Occurrences. - Bulla section, Dolomites, Italy, Bulla Member, Bellerophon Formation, Changxingian (Crasquin et al. 2008b); Meishan section, Changxing Formation, Baoqing and Meishan Members (Shi \& Chen 1987); sample 08LO02-10, Tham Nam Maholan section, Nam Maholan Formation, Loei Province, northeastern Thailand, Early Permian; samples 07PB04-2 and 07PB04-5, Nong Phai section, Pha Nok Khao Formation, Phetchabun Province, central Thailand, Early Permian.

Dimensions. $-\mathrm{H}=0.280-0.594 \mathrm{~mm}, \mathrm{~L}=0.532-1.150 \mathrm{~mm}$, $\mathrm{H} / \mathrm{L}=0.48-0.52$.

\section{REMARKS}

The specimens are attributed to Bairdia deweveri Crasquin, 2010 described from the Late Permian of Italy, and South China (see synonymy) due to the angular dorsal outline of both valves, and $\mathrm{AB}$ angular, pointing upward and located almost as high as DB. PB is tapering and located very low, nearly at VB.
Bairdia cf. deweveri Crasquin, 2010

(Fig. 11A, B)

Bairdia deweveri Crasquin in Crasquin, Forel, Feng, Yuan, Baudin \& Collin et al. 2010a: 342, figs 7O-T.

MATERial EXAMINED. - Nine incomplete carapaces.

Occurrences. - Sample 07PB04-2, Nong Phai section, Pha Nok Khao Formation, Phetchabun Province, Early Permian; sample 07LB05-C2, Ta Kli section, Tak Fa Formation, Nakhon Sawan province, Central Thailand, Early Permian.

Dimensions. $-\mathrm{H}=0.40-0.55 \mathrm{~mm}, \mathrm{~L}=0.77-0.99 \mathrm{~mm}, \mathrm{H} / \mathrm{L}=$ $0.48-0.54$.

\section{REMARKS}

The present species can be compared to Bairdia deweveri Crasquin, 2010 from the latest Permian of Meishan section, South China (Crasquin et al. 2010a: 19, pl. 7, figs 15-20); however, AB of $B$. deweveri has smaller radius of curvature than in our specimens.

\section{Bairdia broutini Crasquin, 2010} (Fig. 11D-E)

Bairdia broutini Crasquin in Crasquin, Forel, Feng, Yuan, Baudin \& Collin et al. 2010a: 340, 342, figs 9O-T.

Rectobairdia tantilla Kummerow, 1953: 52, pl. 5, figs 8a, b. — Shi \& Chen 1987: 41, pl. 5, figs 3, 4, 7, 8 (?5, 6, 9, 10); 2002: 71, pl. 8, figs 5-7, pl. 9, figs 6-9.

MATERIAL EXAMINED. - Three complete and two incomplete carapaces.

OcCurRences. - Meishan section, South China, latest Permian (Crasquin et al. 2010a); sample 07LB05-2, Ta Kli section, Tak Fa Formation, Nakhon Sawan Province, Central Thailand, Early Permian; sample 07LB04-13, Phu Lam Yai section, Tak Fa Formation, Nakhon Sawan Province, Central Thailand, Early Permian.

DiMENSIONS. $-\mathrm{H}=0.43-0.54 \mathrm{~mm}, \mathrm{~L}=0.71-0.88 \mathrm{~mm}, \mathrm{H} / \mathrm{L}=0.60-0.62$.

Bairdia pierrevalentini Crasquin, 2010

(Fig. 11G-H)

Bairdia pierrevalentini Crasquin in Crasquin, Forel, Feng, Yuan, Baudin \& Collin et al. 2010a: 347, 348, figs 9A-D.

MATERial EXAMined. - Two incomplete carapaces.

OCCurrences. - Meishan section, South China, latest Permian (Crasquin et al. 2010a); sample 07PB04-5, Nong Phai section, Pha Nok Khao Formation, Phetchabun Province, Central Thailand, Early Permian.

DiMENSIONS. - $\mathrm{H}=0.45-0.46 \mathrm{~mm}, \mathrm{~L}=0.74-0.77 \mathrm{~mm}, \mathrm{H} / \mathrm{L}=0.58-0.62$.

\section{Bairdia deducta deducta Zalányi, 1974}

(Fig. 11J-L)

Bairdia deducta deducta Zalányi, 1974: 196, 197, pl. 12, fig. 1a-c. Kozur 1985a: 62 (not illustrated). - Crasquin-Soleau \& Baud 1998: pl. 8, figs 10-13, 16. 

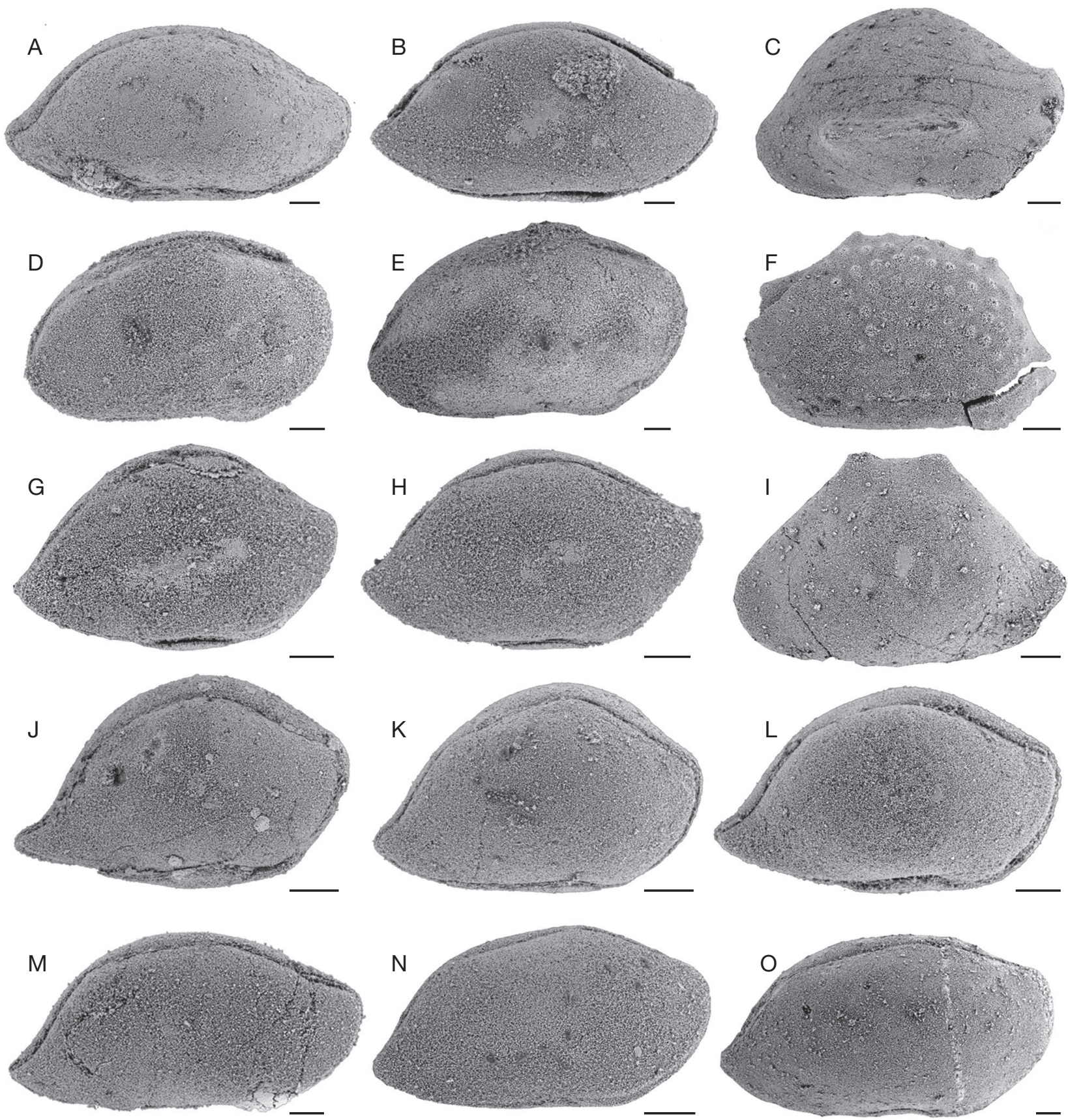

FIG. 11. - Ostracods from Indochina Block, Central Thailand. All specimens are stored at the Suranaree University of Technology Collections (Nakhon Ratchasima, Thailand) numbers SUT-09-xxxx. A, B, Bairdia cf. deweveri Crasquin, 2010; A, right lateral view of a complete carapace, SUT-09-2129, sample 07PB03-3; B, right lateral view of a complete carapace, SUT-09-2130, sample 07PB03-3; C, Bairdia sp. B, external view of a right valve, SUT-09-2295, sample 07PB08-2; D, E, Bairdia broutini Crasquin, 2010; D, right lateral view of a complete carapace, SUT-09-2112, sample 07LB05-2; E, right lateral view of a complete carapace, SUT-09-2116, sample 07LB04-13; F, Bairdia sp. C, left lateral view of a complete carapace, SUT-09-2296, sample 07PB06-5; G, H, Bairdia pierrevalentini Crasquin, 2010; G, right lateral view of a complete carapace, SUT-09-2256, sample 07PB04-5; H, right lateral view of a complete carapace, SUT-09-2257, sample 07PB04-5; I, Petasobairdia sp., left lateral view of a complete carapace, SUT-09-2577, sample 07PB08-3; J-L, Bairdia deducta deducta Zalanyi, 1974 sensu Kozur 1985; J, right lateral view of a complete carapace, SUT-09-2172, sample 07PB05-2; K, right lateral view of a complete carapace, SUT-09-2177, sample 08LB01-6; L, right lateral view of a complete carapace, SUT-09-2176, sample 08LO02-9; M-O, Bairdia lungtanensis Chen, 1958; M, right lateral view of a complete carapace, SUT-09-2244, sample 07PB03-7; N, right lateral view of a complete carapace, SUT-09-2246, sample 08LO02-9; 0, right lateral view of a complete carapace, SUT-09-2245, sample 08LO02-11. Scale bars: $100 \mu \mathrm{m}$.

Not Bairdia deducta Zalányi, 1974: 196, 197, pl. 12, fig. 1a-c.

Cryptobairdia deducta deducta - Kozur 1985a: pl. 6, fig. 2.

MATERIAL EXAMINED. - Seven complete carapaces.
OCCURRENCES. - Nagyvisnýo Formation, Bükk Mountains, Hungary, Late Permian (Kozur 1985a); Episkopi Formation, late Middle-early Late Permian, Hydra Island, Greece (Crasquin-Soleau \& Baud 1998); sample 08LO02-9, Tham Nam Maholan section, Nam Maholan Formation, Loei Province, northeast Thailand, Early Permian; sam- 
ples 07PB05-2 and 07PB05-3, Ban Naen Sawan I section, Pha Nok Khao Formation, Phetchabun Province, central Thailand, Middle Permian; sample 08LB01-6, Khao Som Phot section, Tak Fa Formation, Lopburi Province, central Thailand, late Middle Permian.

Dimensions. $-\mathrm{H}=0.411-0.511 \mathrm{~mm}, \mathrm{~L}=0.680-0.790 \mathrm{~mm}$, $\mathrm{H} / \mathrm{L}=0.60-0.64$.

\section{Bairdia lungtanensis Chen, 1958} (Fig. 11M-O)

Bairdia lungtanensis Chen, 1958: 224, 246, pl.4, Figs 1-8.

MATERIAL EXAMINED. - Five complete carapaces.

OCCURRENCES. - Lungtan section, Chihsia Limestone, Nanking Province, Early Permian (Chen 1958); samples 08LO02-9 and 08LO02-11, Tham Nam Maholan section, Nam Maholan Formation, Loei province, northeastern Thailand, Early Permian; sample 07PB03-7, Khao Kana section, Pha Nok Khao Formation, Phetchabun province, central Thailand, Early Permian; sample 07LB05-1, Ta Kli section, Tak Fa Formation, Middle Permian, Nakhon Sawan province, Central Thailand.

Dimensions. $-\mathrm{H}=0.39-0.75 \mathrm{~mm}, \mathrm{~L}=0.71-1.40 \mathrm{~mm}, \mathrm{H} / \mathrm{L}=$ $0.51-0.54$.

\section{REMARKS}

The specimens recovered from central Thailand are of different sizes; the specimen shown in Fig. $11 \mathrm{M}$ is comparable with illustration of Chen (1958: fig. 1, pl. 4) but others are somewhat different that may suggest ontogenic morphological changes.

\section{Bairdia menardensis Harlton, 1929}

(Fig. 12D-F)

Bairdia menardensis Harlton, 1929b: 158, pl. 8, figs 1a-d. - Chen 1958: 248, 249, pl. 3, figs 1-7, 13.

MATERIAL EXAMINED. - Ten complete carapaces.

OCCURRENCES. - Lungtan section, Nanking Province, Early Permian (Chen 1958); samples 07LB05-A3, 07LB05-B1, 07LB05-D3, Ta Kl section, Tak Fa Formation, Nakhon Sawan Province, central Thailand, Early Permian; sample 08LB01-6, Khao Som Phot section, Tak Fa Formation, Lopburi Province, central Thailand, Middle Permian.

DiMENSIONS. $-\mathrm{H}=0.60-0.78 \mathrm{~mm}, \mathrm{~L}=0.95-1.17 \mathrm{~mm}, \mathrm{H} / \mathrm{L}=0.63$

\section{REMARKS}

Sohn (1960) considered Bairdia menardensis as a junior synonym of B. grahamensis Harlton, 1928. The figured specimens of B. grahamensis (Sohn 1960: 45, pl. 1, figs 9-10, 15, 16) are different from our specimens. However, the characters such as subrhombohedral carapace, highly arched DB, narrowly rounded $\mathrm{AB}$ and $\mathrm{PB}$, and upturned $\mathrm{PB}$ agree well with characters of Bairdia menardensis described by Chen (1958) from the Early Permian of China. In the studied specimens, AVB is slightly convex and maximum of curvature of $A B$ is located at or just above mid $\mathrm{H}$.
Bairdia piscariformis Chen, 1958

(Fig. 13K-L)

Bairdia piscariformis Chen, 1958: 245, pl. 4, figs 9, 10.

MATERIAl EXAMINED. - Four complete carapaces.

OcCurrences. - Lungtan section, Chihsia Limestone, Nanking Province, Early Permian (Chen 1958); sample 08LO02-1, Tham Nam Maholan section, Nam Maholan Formation, Loei province, northeastern Thailand, Early Permian; sample 07LB09-2, Khao Phu Chongkho section, Tak Fa Formation, Phetchabun province, central Thailand, Middle Permian.

DiMENSIONS. $-\mathrm{H}=0.39-0.63 \mathrm{~mm}, \mathrm{~L}=0.79-1.30 \mathrm{~mm}, \mathrm{H} / \mathrm{L}=0.48$.

\section{REMARKS}

Bairdia piscariformis, from the Early Permian of China (Chen 1958) is characterized by a long carapace, broadly arched dorsal outline, rounded $A B$ with medium radius of curvature, rounded $\mathrm{PB}$ with small radius of curvature, and straight $\mathrm{ADB}$, $\mathrm{DB}, \mathrm{PDB}$ at RV.

Genus Lobobairdia Kollmann, 1963

TYPE SPECIES. - Lobobairdia salinaria Kollmann, 1963 by original designation.

\section{Lobobairdia ventriconcava (Chen, 1958)}

(Fig. 12G-I)

Bairdia ventriconcava Chen, 1958: 243, pl.3, figs 8-12

Lobobairdia ventriconcava - Chen \& Bao 1986: 118, pl. 2, figs 1-4. Shi \& Chen 2002: 80, pl. 14, figs 1-4, 12-14.

Material EXAmined. - Eight complete and four incomplete carapaces.

OCcurrences. - Jiangsu Province, Early Permian (Chen 1958; Chen \& Bao 1986); Guangxi Province, South China, Late Permian (Shi \& Chen 2002); sample 07PB04-2, Nong Phai section, Pha Nok Khao Formation, Phetchabun Province, central Thailand, Early Permian; samples 07PB05-2, 07PB07-1 and 07PB07-3, Ban Naen Sawan I and II sections, Tak Fa Formation, Phetchabun Province, central Thailand, Middle Permian; sample 08PB02-13, Phu Prathat section, Tak Fa Formation, Phetchabun Province, central Thailand, Middle Permian.

DiMENSIONS. - $\mathrm{H}=0.48-0.61 \mathrm{~mm}, \mathrm{~L}=0.76-0.92 \mathrm{~mm}, \mathrm{H} / \mathrm{L}=0.63-0.66$.

Genus Petasobairdia Chen, 1982

TYPe SPECIES. - Petasobairdia bicornuta Chen, 1982 by original designation.

\section{Petasobairdia subnantongensis Chen, 1987}

(Fig. 15A-C)

Petasobairdia subnantongensis Chen in Shi \& Chen, 1987: 47, pl. 8, figs $1-4$, pl. 19, figs 8, 9. - Shi \& Chen 2002: 75, pl. 14, figs 1214. - Crasquin et al. 2010a: 350, figs 13F-H. 

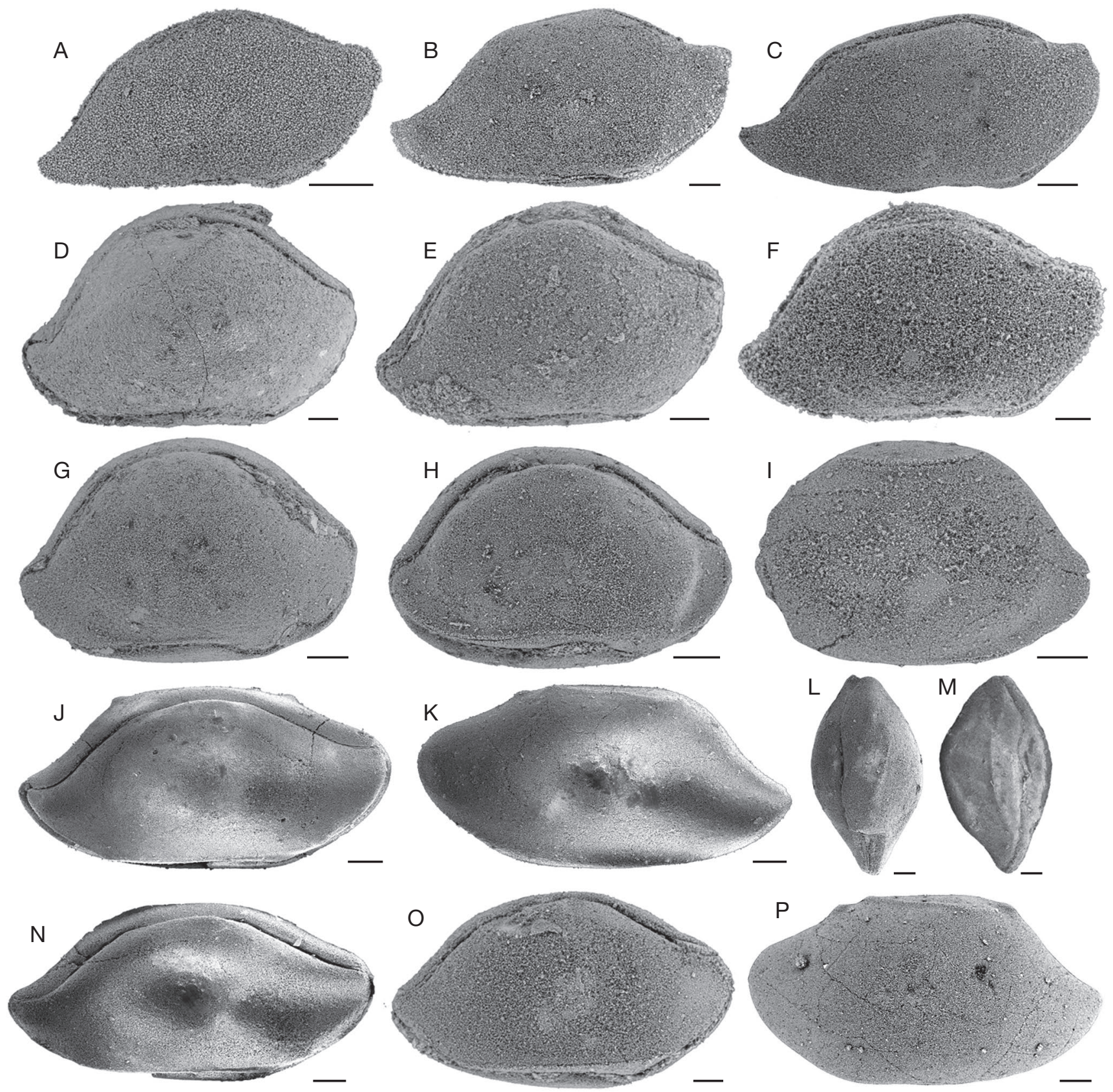

FIG. 12. - Ostracods from Indochina Block, Central Thailand. All specimens are stored at the Suranaree University of Technology Collections (Nakhon Ratchasima, Thailand) numbers SUT-09-xxxx. All the specimens are represented by complete carapaces. A-C, Bairdia deweveri Crasquin, 2010; A, right lateral view, SUT-092165, sample 07PB04-2; B, right lateral view, SUT-09-2161, sample 07PB04-2; C, right lateral view, SUT-09-2169, sample 08LO02-10; D-F, Bairdia menardensis Harlton sensu Chen (1958); D, right lateral view, SUT-09-2140, sample 07LB05-B1; E, right lateral view, SUT-09-2147, sample 07LB05-D3; F, right lateral view, SUT-09-2148, sample 08LB01-6; G-I, Lobobairdia ventriconcava (Chen, 1958); G, right lateral view, SUT-09-2539, sample 07PB05-2; H, right lateral view, SUT09-2540, sample 07PB05-2; I, left lateral view, SUT-09-2541, sample 07PB07-3; J-P, Petasobairdia campbelli Chitnarin, n. sp.; J, holotype, right lateral view, SUT-09-2568, sample 07LB05-5; K, paratype, left lateral view, SUT-09-2571, sample 07LB05-5; L, dorsal view, SUT-09-2572, sample 07LB05-5; M, ventral view, SUT-09-2573, sample 07LB05-5; N, paratype, right lateral view, SUT-09-2570, sample 07LB05-5; O, left lateral view, SUT-09-2574, sample 07PB05-5; P, left lateral view, SUT-09-2575, sample 07PB05-5. Scale bars: $100 \mu \mathrm{m}$.

Petasobairdia nantongensis Chen in Chen \& Shi, 1982: 130, pl. 6, figs 7-9 (non figs 1-6). — Crasquin et al. 2008b: pl. 4, figs 16, 17.

MATERIAL EXAMINED. - 23 complete and six incomplete carapaces.

OCCurRences. - Jiangsu Province, latest Permian (Chen \& Shi 1982); Meishan section, Zhejiang Province, latest Permian (Shi \& Chen 1987; Crasquin et al. 2010a); Guangxi Province, South China, Late Permian (Shi \& Chen 2002); sample 07LB05-5, Ta Kli sec- tion, Tak Fa Formation, Nakhon Sawan Province, central Thailand, Early Permian; samples 07PB05-2 and 07PB05-3, Ban Naen Sawan I section, Tak Fa Formation, Phetchabun Province, central Thailand, Middle Permian; sample 08PB03-3, Phu Pra that section, Tak Fa Formation, Phetchabun Province, central Thailand, Middle Permian.

Dimensions. $-\mathrm{H}=0.45-0.62 \mathrm{~mm}, \mathrm{~L}=0.81-0.96 \mathrm{~mm}, \mathrm{H} / \mathrm{L}=$ 0.61-0.64. 
Petasobairdia campbelli Chitnarin, n. sp. (Figs 12J-P; 17)

TYPe MATERIAL. - Holotype, one complete carapace (SUT-092568, Fig. 12F); paratypes, two complete carapaces (SUT-09-2570, Fig. $12 \mathrm{~N}$ and SUT-09-2571, Fig. 12K).

Etymology. - In honour of Dr Hamish Campbell, GNS, New Zealand.

Material EXAMINED. - Nine complete carapaces.

Dimensions. $-\mathrm{H}=0.51-0.69 \mathrm{~mm}, \mathrm{~L}=1.07-1.29 \mathrm{~mm}$, Holotype: $\mathrm{H}=0.57 \mathrm{~mm}, \mathrm{~L}=1.18 \mathrm{~mm}$; Paratype (Fig. $12 \mathrm{~N}): \mathrm{H}=0.51 \mathrm{~mm}, \mathrm{~L}=$ $1.07 \mathrm{~mm}$; Paratype (Fig. 12K): $\mathrm{H}=0.55 \mathrm{~mm}, \mathrm{~L}=1.28 \mathrm{~mm}$ (Fig. 17).

TyPe HORIZON. - Sample 07LB05-5, Ta Kli section, Tak Fa Formation, Early Permian.

Type LOCAliTy. - Ta Kli section (1519'05”N, 100²2'46”E), Nakhon Sawan Province, central Thailand.

Occurrences. - Sample 07LB05-5, Ta Kli section, Tak Fa Formation, Nakhon Sawan Province, central Thailand, Early Permian; sample 07PB05-5, Ban Naen Sawan I section, Tak Fa Formation, Phetchabun Province, central Thailand, Middle Permian.

Diagnosis. - Species of Petasobairdia with a long carapace, DB of LV nearly horizontal with an horizontal dorsal ridge ended by small spines at both ends, DB of RV convex underlined by a simple ridge, maximum $\mathrm{H}$ located in median region, ventral ridge at both valves.

\section{DESCRIPTION}

Long, subfusiform carapace; dorsal outline angulated, DB of RV convex underlined by a simple ridge, DB of LV nearly horizontal, underlined by a straight dorsal ridge ended by spines at both ends; $\mathrm{ADB}$ of $\mathrm{RV}$ concave, $\mathrm{ADB}$ of LV slightly concave, angle between $\mathrm{AD}$ and $\mathrm{DB}$ is $160^{\circ}$; $\mathrm{AB}$ rounded with small radius of curvature, maximum convexity located above mid $\mathrm{H}$; AVB slightly convex, angle between AVB and VB is $150^{\circ}$; VB straight with a faint ventral ridge both valves; PVB long, slightly convex, making an angle of $155^{\circ}$ with VB; PB tapering, with maximum of convexity located below mid $\mathrm{H}$; PDB slightly concave on $\mathrm{LV}$, concave on $\mathrm{RV}$, angle between $\mathrm{PDB}$ and $\mathrm{DB}$ is $135^{\circ}$; maximum $\mathrm{H}$ all along DB; strong overlap of $\mathrm{LV}$ on $\mathrm{RV}$ along dorsal outline; in dorsal view carapace biconvex flattened laterally.

\section{REMARKS}

Petasobairdia campbelli Chitnarin, n. sp. can be compared to P. levicornuta Chen, 2002 from the latest Permian of Guangxi in South China (Shi \& Chen 2002: 75, pl. 16, figs 1-7, pl. 17, figs 1-6) by its small dorsal ridge on LV with a backward-pointing spine. However, our specimens differ from $P$. levicornuta by the angular dorsal outline, DB of LV horizontal and presence of small spines at both ends of the horizontal dorsal ridge on LV.

\section{Genus Cryptobairdia Sohn, 1960}

TYPE SPECIES. — Bairdia ventricosa Roth \& Skinner, 1930 by original designation,
Cryptobairdia seminalis (Knight, 1928)

(Fig. 15D-F)

Bairdia seminalis Knight, 1928: 320, pl. 43, fig. 2a-d. — Kellett 1934: 127, pl. 15, fig. 2a-c.

Cryptobairdia seminalis - Sohn 1960: 51. - Chen \& Shi 1982: 126, pl. 7, fig. 1-4.

MATERIAL EXAMINED. - Eight complete and two incomplete carapaces.

Occurrences. - Henrietta Formation, Missouri, Middle to Late Pennsylvanian (Knight 1928); Des Moines series and Neva Formation, Kansas, Early Pennsylvanian to Early Permian (Kellett 1934); Nantong section, Jiangsu Province, latest Permian (Chen \& Shi 1982); samples 07PB03-5 and 07PB03-7, Khao Kana section, Pha Nok Khao Formation, Phetchabun Province, central Thailand, Early Permian; sample 07PB04-2, Nong Phai section, Pha Nok Khao Formation, Phetchabun Province, central Thailand, Early Permian.

Dimensions. $-\mathrm{H}=0.31-0.59 \mathrm{~mm}, \mathrm{~L}=0.43-0.84 \mathrm{~mm}, \mathrm{H} / \mathrm{L}=$ $0.70-0.72$.

Cryptobairdia sp.

(Figs 15G; 16G-I)

MATERIAL EXAMINED. — Four complete and three incomplete carapaces

OCCURRENCES. - Samples 08LO07-1 and 08LO07-10, Sak Chai Quarry section, Pha Nok Khao Formation, Chaiyaphum Province, northeastern Thailand, Early Permian; sample 07LB05-2, Ta Kli section, Tak Fa Formation, Nakhon Sawan Province, central Thailand, Early Permian; samples 07PB03-1, 07PB03-7 Khao Kana section, Pha Nok Khao Formation, Phetchabun province, central Thailand, Early Permian; sample 07PB05-6, Ban Naen Sawan I section, Tak Fa Formation, Phetchabun province, central Thailand, Middle Permian.

Dimensions. $-\mathrm{H}=0.30-0.75 \mathrm{~mm}, \mathrm{~L}=0.57-1.11 \mathrm{~mm}, \mathrm{H} / \mathrm{L}=$ 0.61-0.64.

\section{REMARKS}

The specimens can be compared to Cryptobairdia seminalis (Knight, 1928) (see above) by the subelliptical outline and the wide $A B$; however, some differences are recognized such as the longer $\mathrm{ADB}$ of the former species and position of maximal $\mathrm{H}$ which is at mid $\mathrm{L}$ for the former species and in front of mid $\mathrm{L}$ for the latter species.

Genus Bairdiacypris Bradfield, 1935

TYPe SPECIES. - Bairdiacypris deloi Bradfield, 1935 by original designation.

Bairdiacypris longirobusta Chen, 1958

(Fig. 15M)

Bairdiacypris longirobusta Chen, 1958: 255, pl. 7, figs 1-3. Chen \& Shi 1982: 136, pl. 10, figs 12-18. — Shi \& Chen 1987: pl. 12, figs 21, 22; 2002: pl. 21, figs 4-7.

MATERIAL EXAMined. - One complete carapace. 

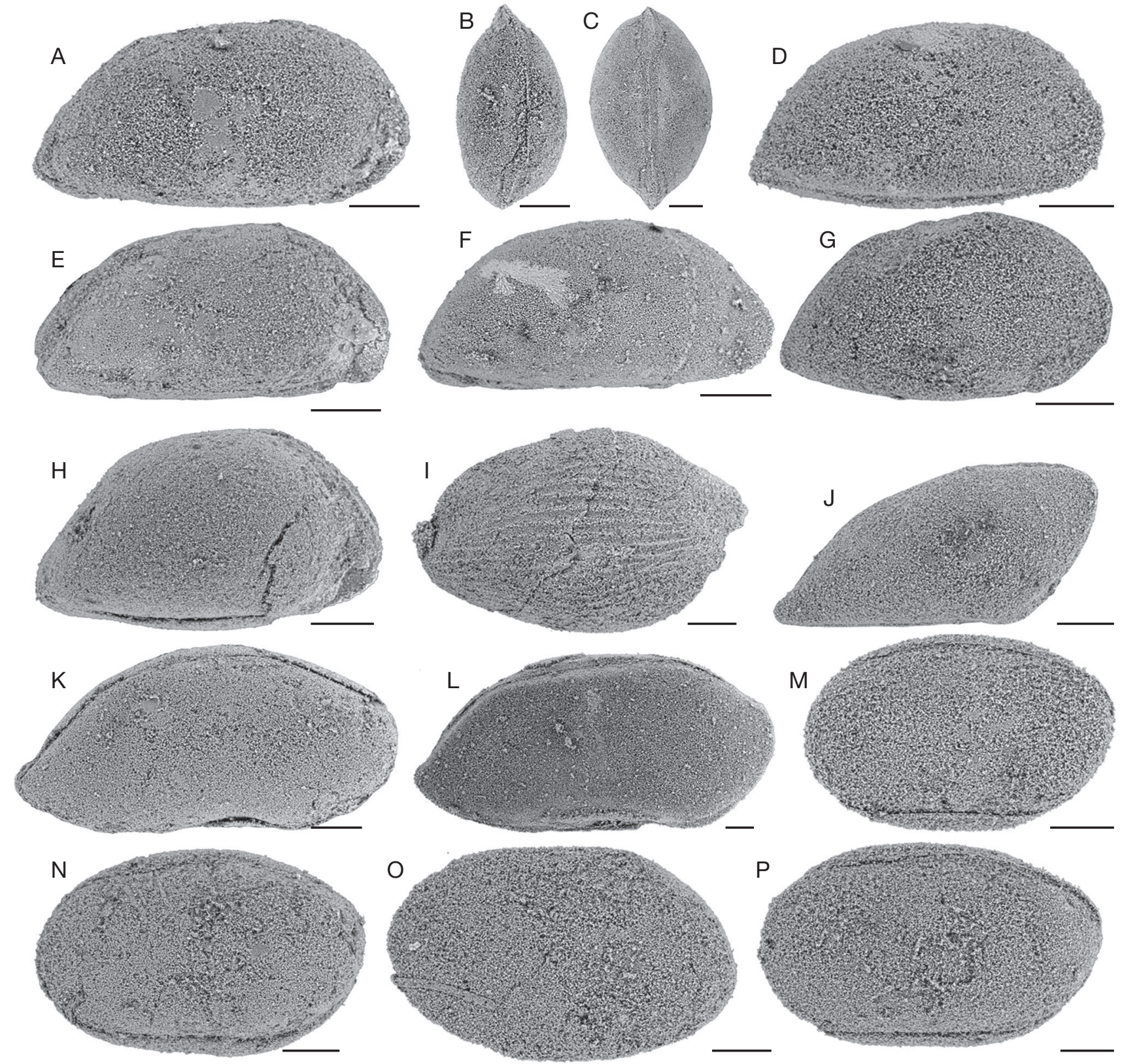

FIG. 13. - Ostracods from Indochina Block, Central Thailand. All specimens are stored at the Suranaree University of Technology Collections (Nakhon Ratchasima, Thailand) numbers SUT-09-xxxx. All the specimens are represented by complete carapaces. A-H, Liuzhinia naramasei Chitnarin, n. sp.; A, holotype, right lateral view, SUT-09-2071, sample 07LB09-1; B, dorsal view, SUT-09-2070, sample 07LB09-1; C, ventral view, SUT-09-2081, sample 07LB05-A2; D, right lateral view, SUT-092080, sample 07LB05-A2; E, paratype, right lateral view, SUT-09-2076, sample 07PB03-5; F, right lateral view, SUT-09-2086, sample 07LB05-B2; G, right lateral view, SUT-09-2095, sample 07LB09-2; H, paratype, right lateral view, SUT-09-20742074, sample 08L01-1; I, ?Pseudocanthoscapha sp., right lateral view, SUT-09-2647, sample 08LO07-2; J, Bohlenatia sp., right lateral view, SUT-09-2491, sample 08LO02-1; K, L, Bairdia piscariformis Chen, 1958; K, right lateral view, SUT-09-2299, sample 07LB09-1; L, right lateral view, SUT-09-2298, sample 08LO02-1; M-P, Cavellina sp.; M, right lateral view, SUT-09-2494, sample 07PB03-3; N, right lateral view, SUT-09-2493, sample 07PB03-3; O, left lateral view, SUT-09-2506, sample 07PB03-3; P, right lateral view, SUT-09-2495, sample 07PB03-3. Scale bars: 100 $\mu$ m.

OCCURRENCES. - Kwanshan and Lungtan sections, Chihsia Limestone, Nanking Province, Early Permian (Chen 1958); Nantong section, Jiangsu Province, latest Permian (Chen \& Shi 1982); Meishan section, Zhejiang Province, latest Permian (Shi \& Chen 1987); Matan and Pingding sections, Guangxi. South China, latest Permian (Shi \& Chen 2002); sample 07LB05-5, Ta Kli section, Tak Fa Formation, Nakhon Sawan Province, central Thailand, Early Permian.

Dimensions. $-\mathrm{H}=0.58 \mathrm{~mm}, \mathrm{~L}=1.36 \mathrm{~mm}, \mathrm{H} / \mathrm{L}=0.42$.
Bairdiacypris deloi Bradfield, 1935

(Fig. 16B)

Bairdiacypris deloi Bradfield, 1935: 93, pl. 7, figs 8a, b, 9a, b. Cooper 1946: 53, pl. 4, figs 22, 23. - Cordell 1952: 95, pl. 19, figs 19, 20. - Sohn 1960: 58, pl. 2, fig. 29; pl. 3 figs 1-5. - Ishizaki 1964: 154, pl. 16, fig. 12. — Lethiers et al. 1989: pl. 2, fig. 9. Crasquin-Soleau \& Baud 1998: pl. 3, fig. 15.

Material eXAmined. - One complete carapace. 

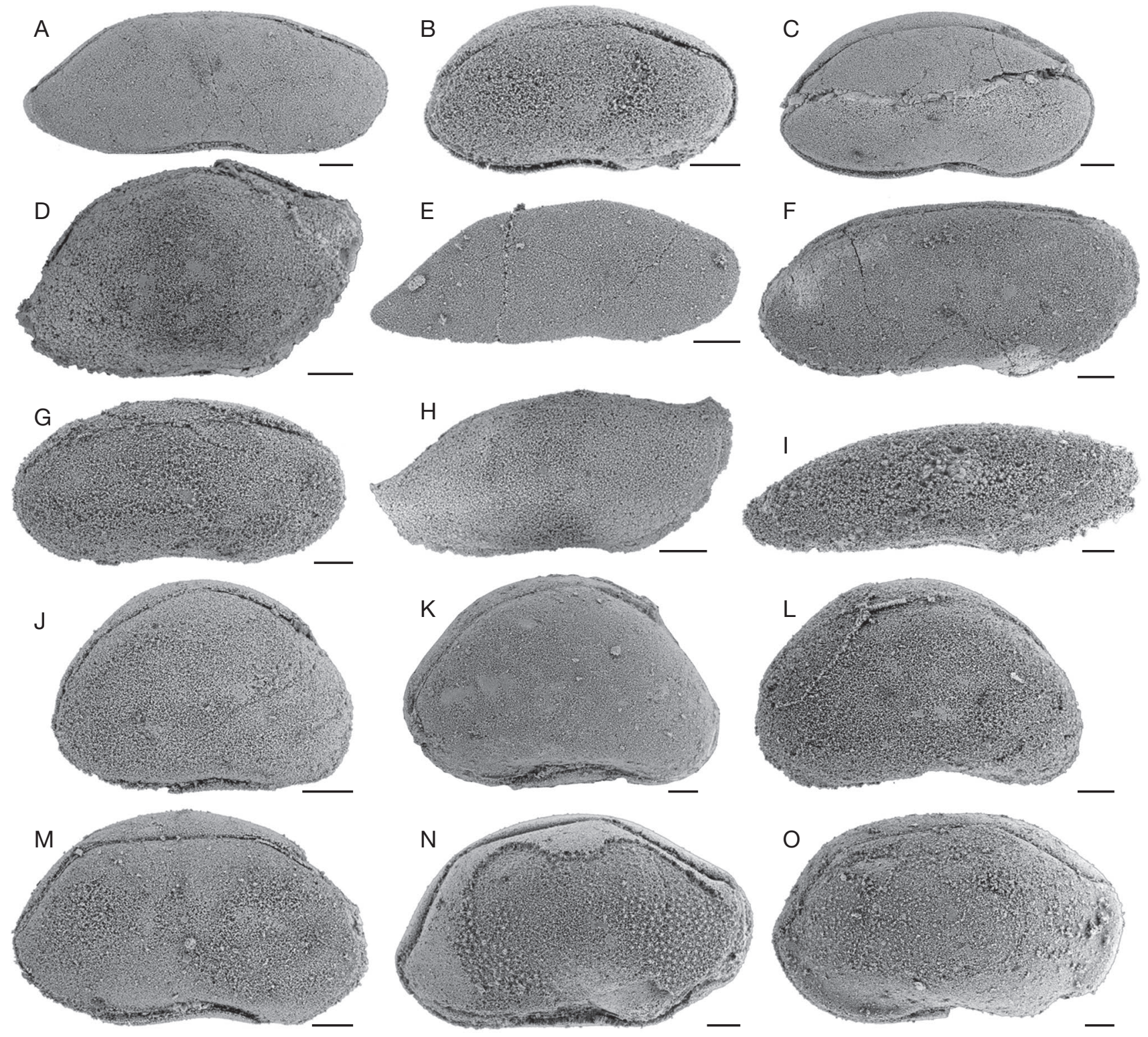

FIG. 14. - Ostracods from the limestones of the Indochina Block, Central Thailand. All specimens are stored at the Suranaree University of Technology Collections (Nakhon Ratchasima, Thailand) numbers SUT-09-xxxx. All the specimens are complete carapaces in right laeral view. A, Bairdiacypris sp. A, SUT-09-2340, sample 07PB05-3. B, C, Bairdiacypris sp. B; B, SUT-09-2341, sample 08LB01-2; C, SUT-09-2351, sample 07LB04-4; D, Bairdia sp. I, SUT-09-2314, sample 07LB04-13; E, Bairdiacypris sp. C, SUT-09-2352, sample 07PB08-3; F, Fabalicypris sp. A, SUT-09-2525, sample 08PB01-2; G, Fabalicypris sp. B, SUT-09-2526, sample 08LB01-3; H, Bairdia sp. J, SUT-09-2315, sample 08LO07-10; I, Bairdiidae indet. sp. A, SUT-09-2492, sample 08LB01-6; J-L, Silenites sureeae Chitnarin, n. sp.; J, paratype, SUT-09-2648, sample 07PB04-5; K, holotype, SUT-09-2650, sample 07LB05-D3; L, SUT-09-2661, sample 08LO02-10; M, Silenites sp., SUT09-2647, sample 07PB05-5; N, O, Kempfina sp.; N, SUT-09-2534, sample 08LB01-2; O, SUT-09-2536, sample 08LO01-4. Scale bars: 100 4 m.

OCCURRENCES. - Pennsylvanian of Western USA (Bradfield 1935; Cooper 1946; Cordell 1952; Sohn 1960); Middle Permian of northeast Japan, (Ishizaki 1964); Middle Permian of Tunisia (Lethiers et al. 1989); Late Permian of Hydra Island, Greece, (Crasquin-Soleau \& Baud 1998); sample 07PB05-6, Ban Naen Sawan I section, Tak Fa Formation, Phetchabun Province, central Thailand, Middle Permian.

Dimensions. $-\mathrm{H}=0.49 \mathrm{~mm}, \mathrm{~L}=1.09, \mathrm{H} / \mathrm{L}=0.45$.

\section{Genus Fabalicypris Cooper, 1946}

TYPE SPECIES. - Fabalicypris wileyensis Cooper, 1946 by original designation.

\section{Fabalicypris hathaithipae Chitnarin, n. sp.}

(Figs 16C-F; 18)

TYPE MATERIAL. - Holotype, one complete carapace (SUT-092341, Fig. 16C); paratypes, two complete carapaces (SUT-09-2348, Fig. 16D and SUT-09-2349, Fig. 16E).

Etymology. - In honour of Assistant Professor Dr Hathaithip Tassanapak, Department of Biology, Mahasarakham University, Thailand.

MATERIAL EXAMINED. - Twenty complete carapaces. 

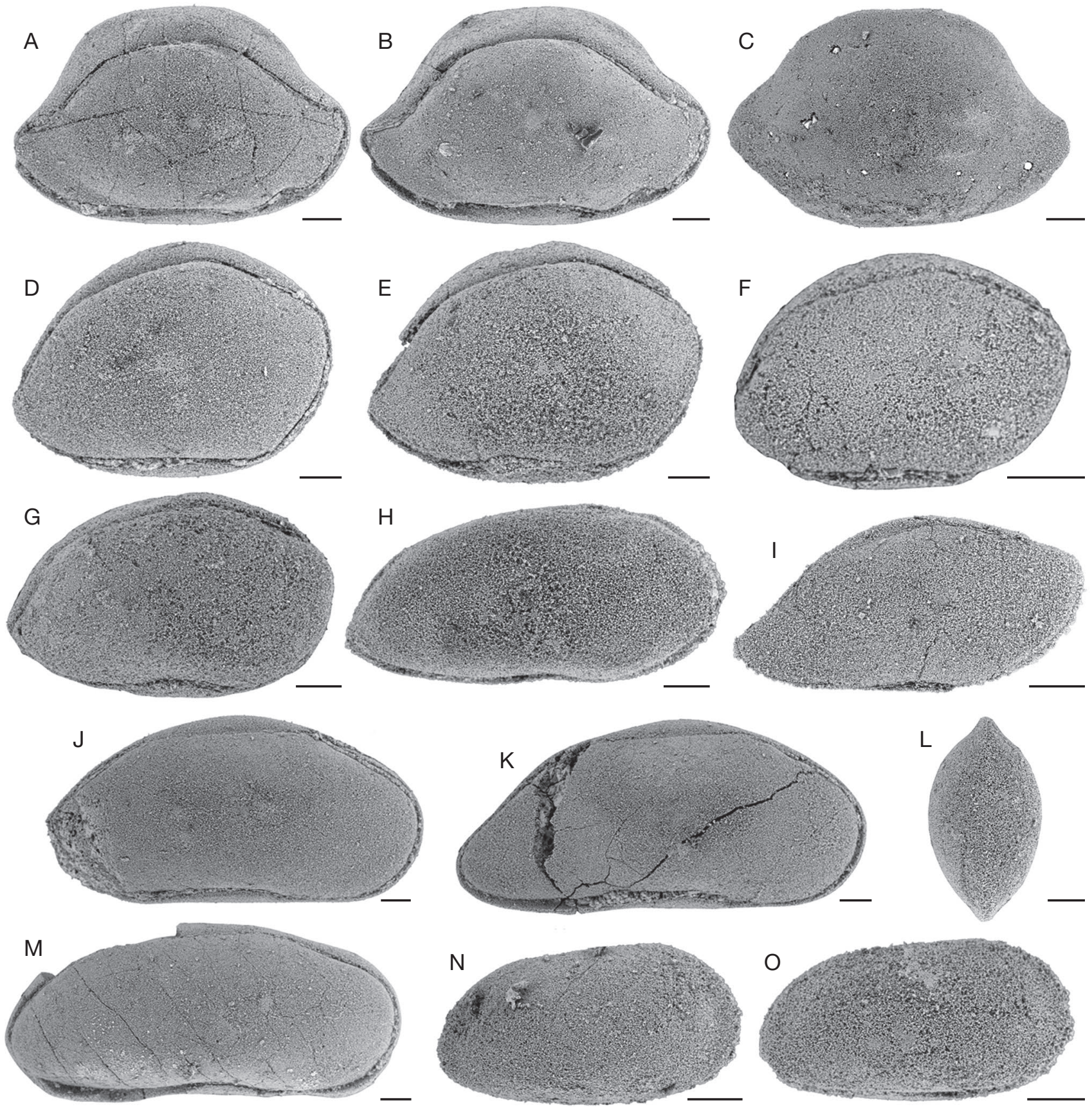

FIG. 15. - Ostracods from Indochina Block, Central Thailand. All specimens are stored at the Suranaree University of Technology Collections (Nakhon Ratchasima, Thailand) numbers SUT-09-xxxx. All the specimens are represented by complete carapaces. A-C, Petasobairdia subnantongensis Chen, 1987; A, right lateral view, SUT-09-2578, sample 07PB05-2; B, right lateral view, SUT-09-2583, sample 07PB05-3; C, left lateral view, SUT-09-2581, sample 07PB05-2. D-F, Cryptobairdia seminalis (Knight) sensu Shi \& Chen, 1982; D, right lateral view, SUT-09-2507, sample 07PB04-2; E, right lateral view, SUT-09-2508, sample 07PB04-2; F, right lateral view, SUT-09-2511, sample 07LB05-5. G, Cryptobairdia sp., right lateral view, SUT-09-2515, sample 07PB03-17. H, Fabalicypris sp. C, right lateral view, SUT-09-2527, sample 07LB07-17. I, Bairdia sp. K, right lateral view, SUT-09-2317, sample 08LO07-8. J, K, Bairdiacypris sp. D; J, right lateral view, SUT-09-2355, sample 07PB05-3; K, right lateral view, SUT-09-2353, sample 07PB05-3. L, N, O, Liuzhinia praeantalyaensis Forel, 2010; L, dorsal view, SUT-09-2099, sample 08LO02-5; N, right lateral view, SUT-09-2100, sample 08LO02-5; O, right lateral view, SUT-09-2099, sample 08LO02-5. M, Bairdiacypris longirobusta Chen, 1958, right lateral view, SUT-09-2361, sample 07LB05-5. Scale bars: $100 \mu \mathrm{m}$.

DiMENSIONS. $-\mathrm{H}=0.31-0.46 \mathrm{~mm}, \mathrm{~L}=0.66-1.02 \mathrm{~mm}, \mathrm{H} / \mathrm{L}=0.41$ 0.45 , Holotype: $\mathrm{H}=0.40 \mathrm{~mm}, \mathrm{~L}=0.96 \mathrm{~mm}$; Paratype (Fig. 16D): $\mathrm{H}=0.42 \mathrm{~mm}, \mathrm{~L}=0.92 \mathrm{~mm}$; Paratype (Fig. 16E): $\mathrm{H}=0.41 \mathrm{~mm}$, $\mathrm{L}=0.89 \mathrm{~mm}$ (Fig. 18).

TyPe HORIZON. - Sample 07PB04-2, Nong Phai section, Pha Nok Khao Formation, Asselian-Sakmarian, Early Permian.
TyPe LOCALITY. — Nong Phai section (1601'06”N, 10058'59"E), Pha Nok Khao Formation, Phetchabun Province, central Thailand.

Occurrences. - Sample 07PB04-2, Nong Phai section, Pha Nok Khao Formation, Phetchabun province, central Thailand, Early Permian; sample 07LO07-8, Sak Chai Quarry section, Pha Nok Khao Formation, Chaiyaphum Province, northeastern Thailand, Early Permian; sample 07LB05-5, Ta Kli section, Tak Fa Formation, 


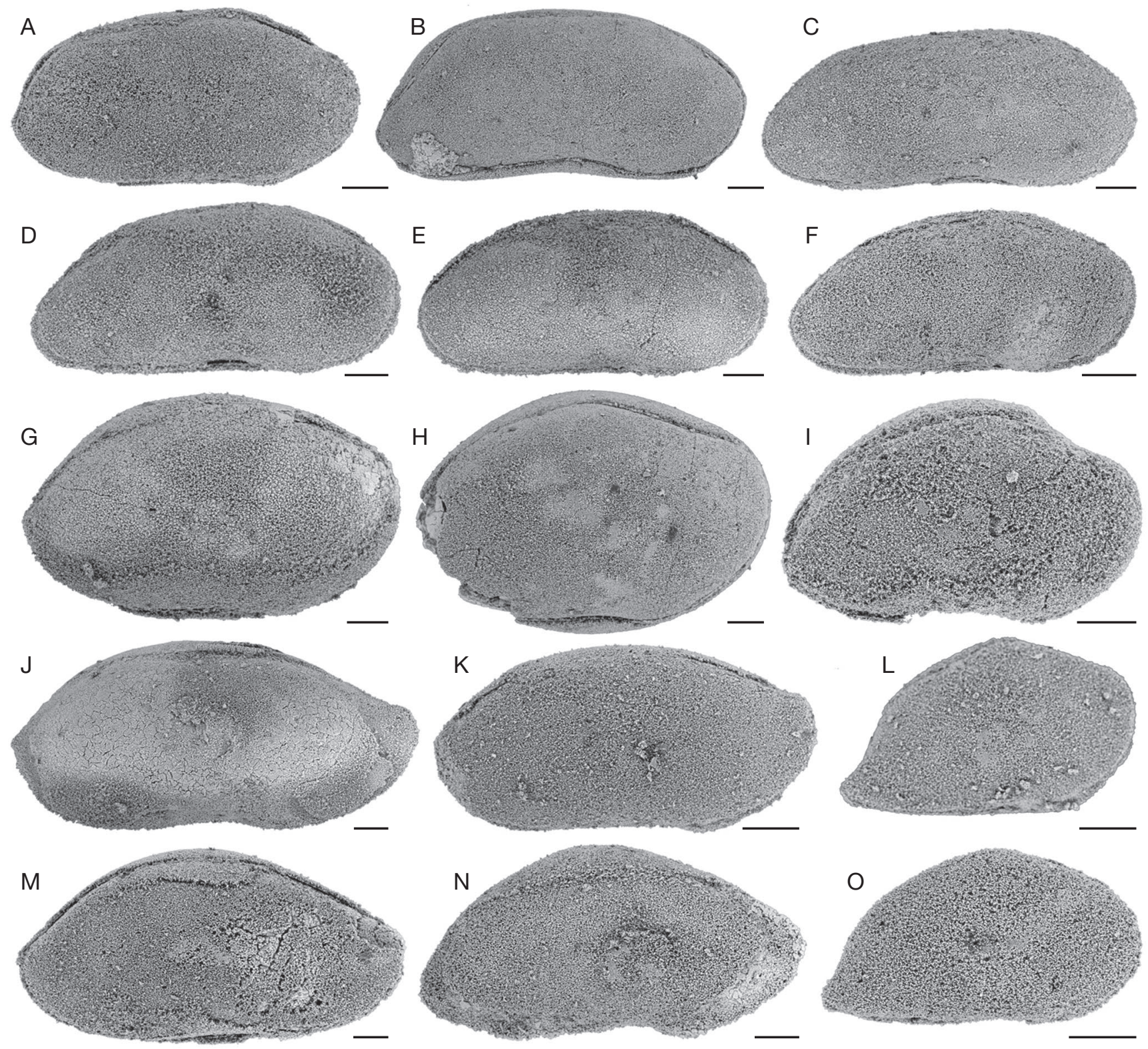

FIG. 16. - Ostracods from the limestones of the Indochina Block, Central Thailand. All specimens are stored at the Suranaree University of Technology Collections (Nakhon Ratchasima, Thailand) numbers SUT-09-xxxx. All specimens are complete carapaces in right lateral view. A, Bairdiacypris sp. E, SUT-09-2357, sample 07PB03-1; B, Bairdiacypris deloi Bradfield, 1935, SUT-09-2360, sample 07PB05-6; C-F, Fabalicypris hathaithipae Chitnarin, n. sp.; C, holotype, SUT-092341, sample 07PB04-2; D, paratype, SUT-09-2348, sample 07PB04-2; E, paratype, SUT-09-2349, sample 07PB04-2; F, SUT-09-2235, sample 08LO07-8; G-I, Cryptobairdia sp., G, SUT-09-2364, sample 08LO07-1; H, SUT-09-2362, sample 07P05-6; I, SUT-09-2363, sample 07P03-1; J, K, Bairdiacypris sp. F; J, SUT-092366, sample 08LO07-2; K, SUT-09-2367, sample 08LO02-11; L, Bairdia sp. L, SUT-09-2369, sample 07PB08-2; M, Bairdiacypris sp. G, SUT-09-2370, sample 07PB03-7; N, Bairdiacypris sp. H, SUT-09-2372, sample 07LB04-13; O, Bairdia sp. M, SUT-09-2373, sample 07LB09-2. Scale bars: 100 4 m.

Nakhon Sawan Province, central Thailand, Early Permian; sample 07LB04-13, Phu Lam Yai section, Tak Fa Formation, Nakhon Sawan Province, central Thailand, Early Permian.

DiAGNOSIS. - Species of Bairdiacypris with maximum of convexity of $\mathrm{AB}$ and $\mathrm{PB}$ located just below mid $\mathrm{H}$, angles of $\mathrm{ADB}$ and $\mathrm{PDB}$ with $\mathrm{DB}$ are of $140^{\circ}$, angles of $\mathrm{ADB}$ and $\mathrm{PDB}$ with $\mathrm{DB}$ are of $160^{\circ}$, maximum $\mathrm{H}$ located in front of mid $\mathrm{L}$.

\section{DESCRIPTION}

Carapace long, subelliptical; dorsal outline broadly arched; DB straight, inclined rearward with an angle of $10^{\circ}$; $\mathrm{ADB}$ short, straight and angle with $\mathrm{DB}$ is $140^{\circ}$; $\mathrm{AB}$ rounded with medium radius of curvature, maximum convexity located below mid $\mathrm{H}$ (at 40-45\%); AVB short and slightly convex; VB slightly concave, angle of AVB and VB is $160^{\circ}$; PVB slightly convex; $\mathrm{PB}$ round with medium radius of curvature, slightly smaller than $\mathrm{AB}$, maximum convexity located at lower $30 \%$ of $\mathrm{H}$; PDB short, straight, angle with DB is $140{ }^{\circ}$ maximum $\mathrm{H}$ located in front of mid L, at antrior third of L; surface smooth.

\section{REMARKS}

Fabalicypris hathaithipae Chitnarin, n. sp. can be compared to Fabalicypris elliptica Chen, 1958 from the Early Permian of Jiangsu Province, Eastern China (Chen 1958: 256, pl. VII, 
figs 7-9) in lateral outline. Fabalicypris elliptica is distinct from the present new species by a more convex AVB and lower position of maximum of convexity of $\mathrm{PB}$ and maximum $\mathrm{H}$ located anteriorly at $30 \%$ of L. Futhermore, the long carapace, straight DB, developed PDB slope exhibited by F elliptica are rather indicative of the genus Bairdiacypris, a revision of this species will be necessary.

\section{Genus Silenites Coryell \& Booth, 1933}

TYPE SPECIES. — Silenites silenus Coryell \& Booth, 1933 by original designation.

\section{Silenites sureeae Chitnarin, n. sp.}

(Figs 14J-L; 19)

TYPE MATERIAL. - Holotype, one complete carapace (SUT-092650, Fig. 14K); paratypes, two complete carapaces (SUT-09-2648, Fig. 14J and SUT-09-2661, Fig. 14L);

ETymology. - In honour of Dr Suree Teerarungsikul, Department of Mineral Resources, Thailand.

MATERIAL EXAMINED. - One incomplete and 13 complete carapaces.

DimENSIONS. - $\mathrm{H}=0.42-0.58 \mathrm{~mm}, \mathrm{~L}=0.60-0.90 \mathrm{~mm}, \mathrm{H} / \mathrm{L}=0.62$ 0.70, Holotype: $\mathrm{H}=0.73 \mathrm{~mm}, \mathrm{~L}=1.08 \mathrm{~mm}$; Paratype (Fig. 14J): $\mathrm{H}=0.42 \mathrm{~mm}, \mathrm{~L}=0.60 \mathrm{~mm}$; Paratype (Fig. $14 \mathrm{~L}$ ): $\mathrm{H}=0.58 \mathrm{~mm}$, $\mathrm{L}=0.90 \mathrm{~mm}$. (Fig. 19).

Type Horizon. - Sample 07LB05-D3, Ta Kli section, Tak Fa Formation, Nakhon Sawan Province, central Thailand, Early Permian.

Type LOCAliTy. — Nong Phai section (1601'06”N, 10058'59”E), Pha Nok Khao Formation, Phetchabun Province, central Thailand.

OCCURRENCES. - Samples 08LO02-5 and 08LO02-10, Nam Maholan section, Nam Maholan Formation, Loei Province, northeast Thailand, Early Permian; sample 07PB04-5, Nong Phai section, Pha Nok Khao Formation, Phetchabun Province, central Thailand, Early Permian; samples 07LB05-D2 and 07LB05-D3, Ta Kli section, Tak Fa Formation, Nakhon Sawan Province, Early Permian; sample 07PB08-3, Ban Nean Sawan II section, Tak Fa Formation, Phetchabun Province, central Thailand, Middle Permian; samples 08LB01-3 and 08LB01-6, Khao Som Phot section, Tak Fa Formation, Lopburi Province, Middle Permian.

DiAGNOSIS. - Species of Silenites with rounded subtriangular carapace, highly arched dorsal outline, short and straight DB of RV, H max is located at mid $\mathrm{L}, \mathrm{AB}$ and $\mathrm{PB}$ of the same size.

\section{DESCRIPTION}

Carapace subtriangular; dorsal outline highly arched; DB short, convex at $\mathrm{LV}$, straight at $\mathrm{RV}$ which inclined at $15-$ $25^{\circ}$ rearward; $\mathrm{ADB}$ long and slightly convex, $\mathrm{AB}$ with large radius of curvature, maximum convexity located at lower fourth of $\mathrm{H}$; AVB short and convex; VB concave; PVB short and convex; $\mathrm{PB}$ round with large radius of curvature, maximum convexity located at lower fourth of $\mathrm{H}$; maximum of curvature of $\mathrm{AB}$ and $\mathrm{PB}$ located at the same level; greatest $\mathrm{H}$ located at mid L; surface smooth; LV larger and overlapping RV at DB.

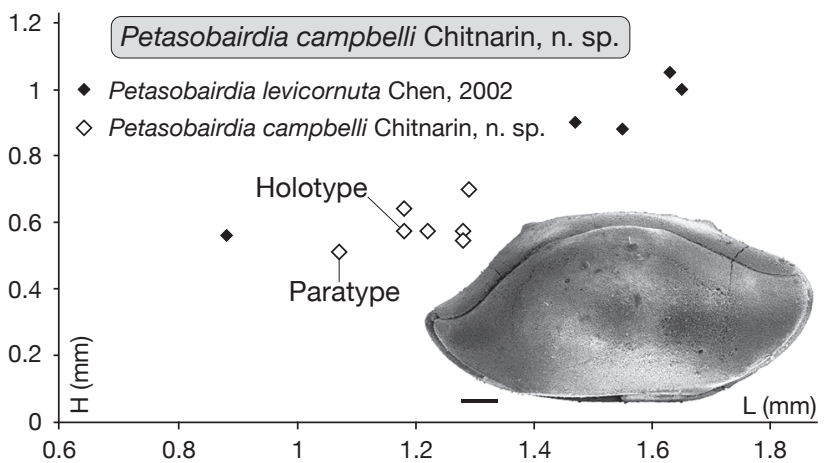

FIG. 17. - Height (H)-length (L) diagram of Petasobairdia campbelli Chitnarin, n. sp. Scale bar: $100 \mu \mathrm{m}$.

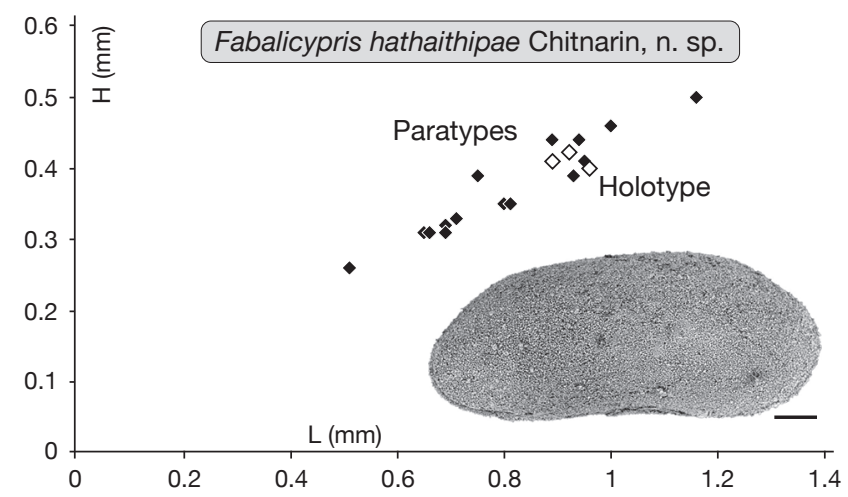

FIG. 18. - Height (H)-length (L) diagram of Fabalicypris hathaithipae Chitnarin, n. sp. Scale bar: $100 \mu \mathrm{m}$.

\section{REMARKS}

Silenites sureeae Chitnarin, n. sp. looks similar to three species from the Early Permian of Jiangsu Province, Eastern China (Chen \& Bao 1986): 1) Silenites testatus Chen, 1958 (in Chen \& Bao 1986: 125, pl. 2, figs 10, 11) but differs in having more rounded DB and maximum $\mathrm{H}$ located at mid L; 2) S. cf. marginiferus (Geis, 1932) sensu Chen \& Bao (1986: 125, 126, pl. 2, fig. 12) but can be differentiated by the straight $\mathrm{DB}$ on RV of $S$. sureeae; and 3) S. unicostus Chen \& Bao, 1986 (Chen \& Bao 1986: 126 , pl. 2, figs 13,14$)$ but it lacks a crest on posteroventral region of RV.

Family ACRATIIDAE Gründel, 1962

Genus Acratia Delo, 1930

TYPE SPECIES. - Acratia typica Delo, 1930 by original designation.

Acratia chongpani Chitnarin, n. sp.

(Figs 21A-G; 22)

Acratina gusevae Kozur, 1985b: 104, pl. 20, fig. 1. - CrasquinSoleau \& Baud 1998: pl. 3, fig. 1. n. syn. 


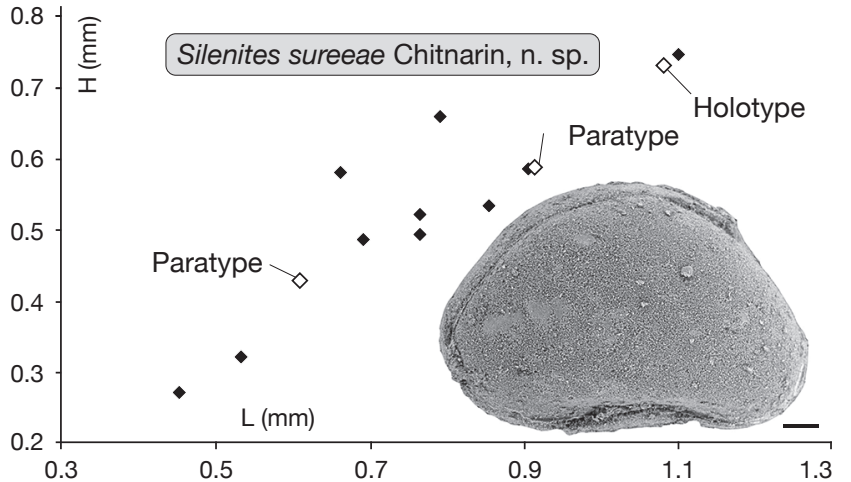

FIG. 19. - Height (H)-length (L) diagram of Silenites sureeae Chitnarin, n. sp. Scale bar: $100 \mu \mathrm{m}$.

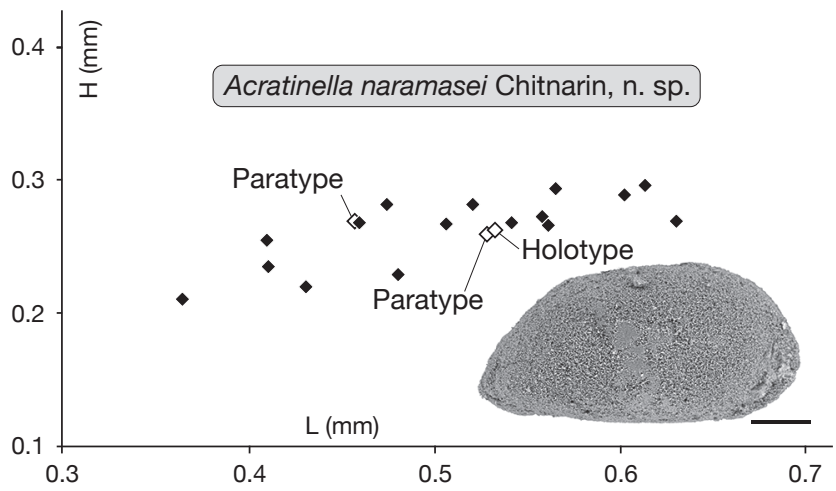

FlG. 20. - Height (H)-length (L) diagram of Acratinella naramasei Chitnarin, n. sp. Scale bar: $100 \mu \mathrm{m}$.

TYPE MATERIAL. - Holotype, one complete carapace (SUT-092006, Fig. 21A); paratypes, two complete carapaces (SUT-09-2001, Fig. 21E and SUT-09-2013, Fig. 21F).

ETyMOLOGY. - In honour of Dr Chongpan Chonglakmani, Thailand.

MATERIAL EXAMINED. - 51 complete carapaces.

DimENSIONS. $-\mathrm{H}=0.25-0.51 \mathrm{~mm}, \mathrm{~L}=0.66-1.18 \mathrm{~mm}, \mathrm{H} / \mathrm{L}=0.43$ 0.53 , Holotype: $\mathrm{H}=0.73 \mathrm{~mm}, \mathrm{~L}=1.08 \mathrm{~mm}$; Paratype (Fig. $21 \mathrm{~F}$ ): $\mathrm{H}=0.42 \mathrm{~mm}, \mathrm{~L}=0.60 \mathrm{~mm}$; Paratype (Fig. 21E): $\mathrm{H}=0.58 \mathrm{~mm}$, $\mathrm{L}=0.90 \mathrm{~mm}$ (Fig. 22).

TyPe HORIZON. - Sample 07LB05-6, Ta Kli section, Tak Fa Formation, Early Permian.

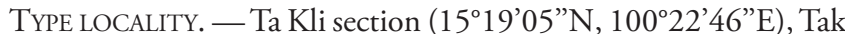
Fa Formation, Nakhon Sawan Province, central Thailand.

OCCURRENCES. — Hydra Island, Greece, late Middle Permian (Crasquin-Soleau \& Baud 1998); sample 08LO07-3, Sak Chai section, Pha Nok Khao Formation, Chiyaphum Province, northeast Thailand, Early Permian; samples 07PB03-2, 07PB03-3 and 07PB03-5, Khao Kana section, Pha Nok Khao Formation, Phetchabun Province, central Thailand, Early Permian; sample 07LB05-6, Ta Kli section, Tak Fa Formation, Nakhon Sawan Province, central Thailand, Early Permian; sample 08LB01-1, Khao Som Phot section, Tak Fa Formation, Lopburi Province, central Thailand, Middle Permian.

Diagnosis. - Species of Acratia with a laterally flattened, long, hook-like $A B$, pointing downward, and located at lower $30 \%$ of $\mathrm{H}$, angle between $\mathrm{AVB}$ and $\mathrm{VB}$ is $150^{\circ}$ and $\mathrm{PB}$ is located at ventral level.

\section{DESCRIPTION}

Carapace long and large; dorsal outline broadly arched; DB slightly convex on LV, straight on RV which gently inclined rearward at $15-20^{\circ}$; $\mathrm{ADB}$ convex and with an angle of $140^{\circ}$ with $\mathrm{DB}$; $\mathrm{PDB}$ convex and with an angle of $140^{\circ}$ with $\mathrm{DB}$; $\mathrm{AB}$ of a hook-like shape, maximum convexity located at lower third of $\mathrm{H}, \mathrm{AB}$ flattened laterally; AVB long and making an angle of $150^{\circ}$ with $\mathrm{VB}$; VB straight to slightly concave; ventral area flat; PVB short in extension of VB; PB bluntly round and located at VB; maximum $\mathrm{H}$ located in front of mid L; surface smooth; LV larger and overlapping RV at ADB and PDB; carapace subfusiform in dorsal view.

\section{REMARKS}

Acratia chongpani Chitnarin, n. sp. can be compared to A. praetypica Posner, 1951 from the Early Carboniferous of Moscow Basin, Russia (Posner 1951: 98, pl. 21, fig. 9) in lateral outline. Differences occur at VB which is blunt in $A$. chongpani but raised upward in $A$. praetypica, and at the angle between $\mathrm{AVB}$ and $\mathrm{VB}$ which is of $150^{\circ}$ in the former species but of $140^{\circ}$ in the latter. Acratina gusevae Kozur sensu Crasquin-Soleau \& Baud 1998 is included to A. chongpani Chitnarin, n. sp. due to the smaller AVB and the shorter PVB than those in Acratina gusevae Kozur, 1985.

\section{Acratia mongkoli Chitnarin, n. sp.}

(Figs 21J-M; 23)

Bairdia galei-Crasquin-Soleau \& Baud 1998: pl. 3, fig. 18 (nec Croneis \& Thurman 1939: 325, pl. 7, fig. 21).

TYPE MATERIAL. - Holotype, one complete carapace (SUT-092058, Fig. 21K); paratypes, two complete carapaces (SUT-09-2063, Fig. 21L and SUT-09-2062, Fig. 21M).

Eтymology. - In honour of Assistant Professor Dr Mongkol Udchachon, Mahasarakham University, Thailand.

MATERIAL EXAMINED. - Ten complete carapaces.

DiMENSIONS. $-\mathrm{H}=0.29-0.54 \mathrm{~mm}, \mathrm{~L}=0.56-1.01 \mathrm{~mm}, \mathrm{H} / \mathrm{L}=0.50$ 0.53 , Holotype: $\mathrm{H}=0.38 \mathrm{~mm}, \mathrm{~L}=0.79 \mathrm{~mm}$; Paratype (Fig. $21 \mathrm{M}$ ): $\mathrm{H}=0.38 \mathrm{~mm}, \mathrm{~L}=0.77 \mathrm{~mm}$; Paratype (Fig. $21 \mathrm{~L}$ ): $\mathrm{H}=0.30 \mathrm{~mm}$, $\mathrm{L}=0.66 \mathrm{~mm}$ (Fig. 23).

Type HORIZON. - Sample 07PB04-2, Nong Phai section, Pha Nok Khao Formation, Asselian-Sakmarian, Early Permian.

TyPe LOCALITY. — Nong Phai section (1601'06”N, 10058'59”E), Pha Nok Khao Formation, Phetchabun Province, central Thailand.

OCCURRENCES. - Early Permian of Hydra Island, Greece (CrasquinSoleau \& Baud 1998); sample 08LO07-2, Sak Chai quarry section, Pha Nok Khao Formation, Chaiyaphum Province, northeast Thailand, Early Permian; sample 07PB03-3, Khao Kana section, Pha Nok Khao Formation, Phetchabun Province, central Thailand, Early Permian; sample 07PB04-2, Nong Phai section, Pha Nok Khao Formation, Phetchabun Province, central Thailand, Early Permian; sample 08PB02-12, Phu Phra That section, Tak Fa Formation, Phetchabun Province, central Thailand, Middle Permian; sample 08LB01-1, Khao Som Phot section, Tak Fa Formation, Lopburi Province, central Thailand, Middle Permian. 

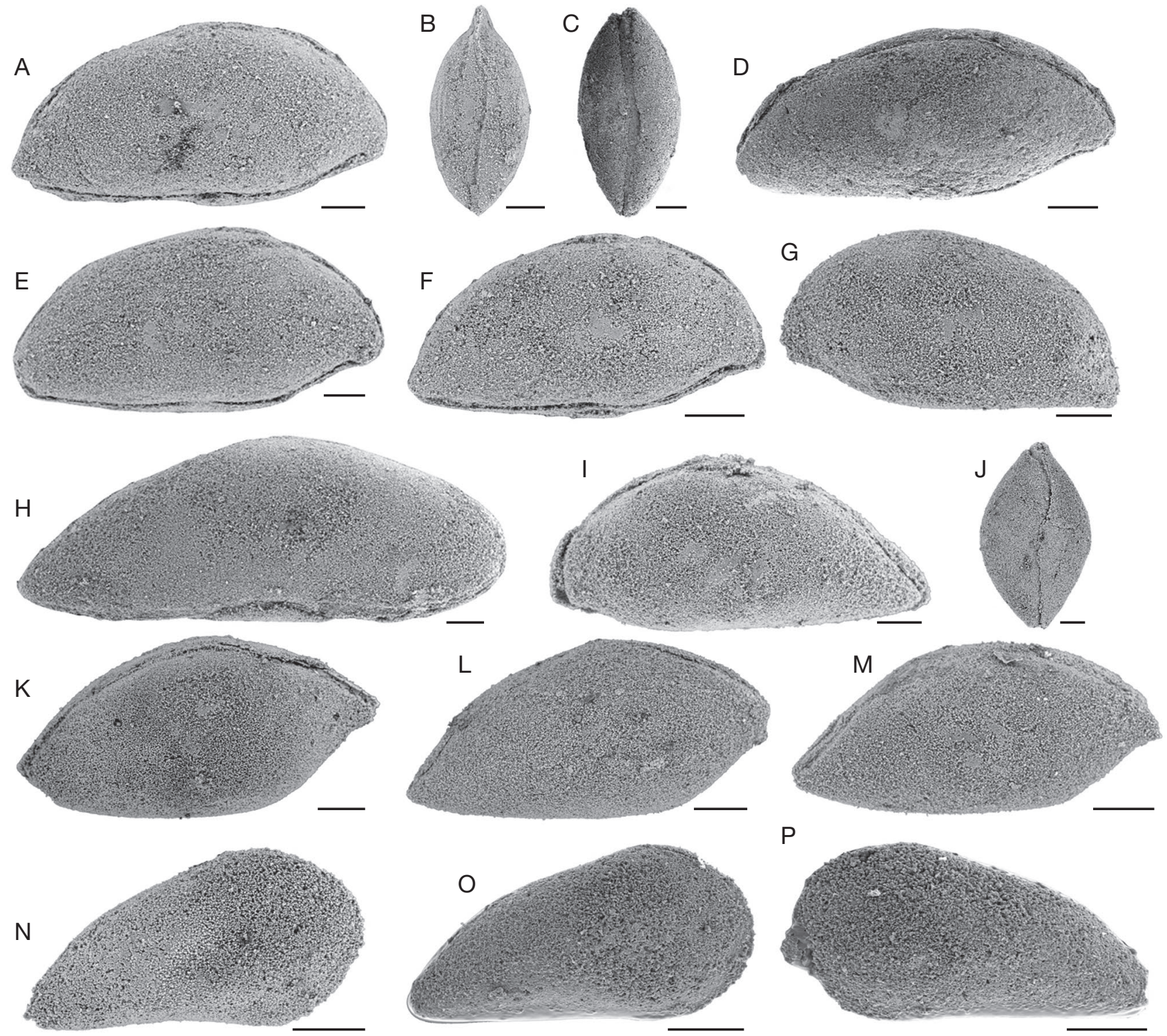

FIG. 21. - Ostracods from the limestones of the Indochina Block, Central Thailand. All specimens are stored at the Suranaree University of Technology Collections (Nakhon Ratchasima, Thailand) numbers SUT-09-xxxx. All the specimens are represented by complete carapaces. A-G, Acratia chongpani Chitnarin, n. sp.; A, holotype, right lateral view, SUT-09-2006, sample 07PB03-3; B, dorsal view, SUT-09-2018, sample 07PB03-5; C, ventral view, SUT-09-2042, sample 07PB03-2; D, right lateral view, SUT-09-2036, sample 07PB03-2; E, paratype, right lateral view, SUT-09-2001, sample 07PB03-5; F, paratype, right lateral view, SUT-09-2013, sample 07PB03-5; G, left lateral view, SUT-09-2020, sample 07PB03-3; H, Bairdiidae indet. sp. B, right lateral view, SUT-09-2567, sample 08LB01-2; I, Acratia sp., left lateral view, SUT-09-2055, sample 08LB01-3; J-M, Acratia mongkoli Chitnarin, n. sp.; J, ventral view, SUT-09-2067, sample 07PB04-2; K, holotype, right lateral view, SUT-09-2058, sample 07PB04-2; L, paratype, right lateral view, SUT-09-2063, sample 07LB09-1; M, paratype, right lateral view, SUT-09-2062, sample 07LB09-1; N-P, Baschkirina cf. huzhouensis Forel, 2010; N, left lateral view, SUT-09-2531, sample 08LB01-1; O, left lateral view, SUT-09-2528, sample 08LB01-1; P, right lateral view, SUT-09-2530, sample 08LB01-1. Scale bars: $100 \mu \mathrm{m}$.

DiAGNOSIs. - Species of Acratia with diamond shape carapace, with laterally flattened and tapering $A B, A B$ points horizontally, AVB makes an angle of $140^{\circ}$ with VB, PVB make an angle of $170^{\circ}$ with VB, PB tapering.

\section{DESCRIPTION}

Carapace with diamond shape in lateral view; dorsal outline broadly arched at LV, angulated at RV; DB of RV inclined at $30^{\circ}$ rearward; $\mathrm{ADB}$ with an angle of $140^{\circ}$ with $\mathrm{DB} ; \mathrm{AB}$ tapering and flattened, pointing horizontally, with maximum of curvature located above mid H; AVB long and straight, making an angle of $140^{\circ}$ with VB; VB straight; ventral area flattened; PVB short, angle of PVB and VB is $170^{\circ}$; PB tapering with maximum of curvature located very low; greatest $\mathrm{H}$ located at mid L; surface smooth; LV larger and overlapping $\mathrm{RV}$ at DB; carapace fusiform in dorsal view.

\section{REMARKS}

Acratia mongkoli Chitnarin, n. sp. can be distinguished from A. chongpani Chitnarin, n. sp. (see above) by the smaller radius of curvature of $\mathrm{AB}$, the tapering $\mathrm{PB}$, and the angle between $\mathrm{AVB}$ and VB. It can be compared with $A$. subfusiformis Wang, 1978 from the Late Permian of South China (Crasquin et al. 2010a: figs 23I-L), however, the $\mathrm{AB}$ of the present species is 
more tapering. It can be compared to A. pulchra Posner, 1951 from the Early Carboniferous of Moscow Basin, Russia (Posner 1951: 98, pl. 21, fig. 8) by the small radius of curvature of $A B$ and $\mathrm{PB}$ but is differentiated by the position of $\mathrm{AB}$ and the angle between AVB and VB. Noteworthy, the specimens documented from the Early Permian of Greece by Crasquin-Soleau \& Baud (1988) have been attributed to the Bairdia galei Croneis \& Thurman, 1939 sensu Chen \& Bao (1986). However, Bairdia galei is not mentioned neither figured by Chen \& Bao (1986).

\section{Acratia? sp.}

(Fig. 21I)

Material EXAMined. - Two complete carapaces.

OCCURRENCES. - Samples 08LB01-1 and 08LB01-3, Khao Som Phot section, Tak Fa Formation, Lopburi Province, central Thailand, Middle Permian.

DimENSIONS. $-\mathrm{H}=0.39-0.40 \mathrm{~mm}, \mathrm{~L}=0.83-0.85 \mathrm{~mm}, \mathrm{H} / \mathrm{L}=0.46$

\section{REMARKS}

Acratia? sp. is characterized by an unusual reverse overlap of RV over LV. The inversion of overlap was observed by Zazzali et al. (2015: fig. 15C, D) on specimens from GuadalupianLopingian boundary of South China. The overlapping of RV over LV can be recognized as a distinct character of the genus Macrocypris Brady, 1867 (Moore 1961: Q207, fig. 146 $1 \mathrm{a}-1 \mathrm{~b})$; however, the studied specimen is assigned here, with doubt, to Acratia due to the presence of acratian beak at AB.

Genus Liuzhinia Zheng, 1976

TYPE SPECIES. — Liuzhinia subovata Zheng, 1976 by original designation.

\section{Liuzhinia praeantalyaensis Forel, 2010}

(Fig. 15L, N, O)

Liuzhinia praeantalyaensis Forel in Crasquin, Forel, Feng, Yuan, Baudin \& Collin, 2010a: 253-255, fig. 23Z-B'.

MATERIAL EXAMINED. - Three complete carapaces.

OCCurRenCes. - Kongtongshan section, Fujian Province, China, Late Permian (Yi 2004); Meishan section, Zhejiang Province, South China, Late Permian (Crasquin et al. 2010a); sample 08LO02-5, Tham Nam Maholan section, Nam Maholan Formation, Loei Province, northeast Thailand, Early Permian.

DiMENSIONS. $-\mathrm{H}=0.29-0.30 \mathrm{~mm}, \mathrm{~L}=0.59-0.59 \mathrm{~mm}, \mathrm{H} / \mathrm{L}=0.50-0.51$

Liuzhinia naramasei Chitnarin, n. sp. (Figs 13A-H; 20)

TYPE MATERIAL. - Holotype, one complete carapace (SUT-092071, Fig. 13A); paratypes, two complete carapaces (SUT-09-2076, Fig. 13E and SUT-09-2074, Fig. 13H).
ETYMOLOGY. - In honour of Mr Naramase Teerarungsigul, Department of Mineral Resources, Thailand.

MATERIAL EXAMINED. - 29 complete carapaces.

Dimensions. $-\mathrm{H}=0.26-0.44 \mathrm{~mm}, \mathrm{~L}=0.52-0.56 \mathrm{~mm}, \mathrm{H} / \mathrm{L}=0.50$ 0.58 , Holotype: $\mathrm{H}=0.26 \mathrm{~mm}, \mathrm{~L}=0.53 \mathrm{~mm}$; Paratype (Fig. 13E): $\mathrm{H}=0.26 \mathrm{~mm}, \mathrm{~L}=0.528 \mathrm{~mm}$; Paratype (Fig. $13 \mathrm{H}): \mathrm{H}=0.27 \mathrm{~mm}$, $\mathrm{L}=0.63 \mathrm{~mm}$ (Fig. 20)

TyPe HORIzON. - Sample 07LB09-1, Khao Phu Chong Kho section, Tak Fa Formation, Kungurian, Early Permian.

TyPe LOCAlity. - Khao Phu Chong Kho section $\left(15^{\circ} 22^{\prime} 45^{\prime \prime} \mathrm{N}\right.$, $100^{\circ} 35^{\prime} 12$ ”E) Tak Fa Formation, Lopburi Province, central Thailand.

OCCURRENCES. - Sample 07PB03-5, Khao Kana section, Pha Nok Khao Formation, Phetchabun Province, central Thailand, Early Permian; sample 08LO07-1, Sak Chai Quarry, Pha Nok Khao Formation, central Thailand, Early Permian; sample 07LB09-2, Khao Phu Chongko section, Tak Fa Formation, Nakhon Sawan Province, central Thailand, Early Permian; samples 07LB05-B1 and 07LB05-B2, Ta Kli section, Tak Fa Formation, Nakhon Sawan Province, central Thailand, Early Permian.

DiagnOSIS. - Species of Liuzhinia with a subtrapezoidal carapace, $\mathrm{ADB}$ and $\mathrm{PDB}$ straight, $\mathrm{AB}$ rounded and laterally flattened, maximum convexity located below mid $\mathrm{H}, \mathrm{PB}$ acuminate and with maximum of curvature located at $\mathrm{VB}$, ventral area flat.

\section{DESCRIPTION}

Carapace small, subtrapezoid; dorsal outline broadly arched; $\mathrm{DB}$ straight and gently inclined to posterior; $\mathrm{ADB}$ and $\mathrm{PDB}$ straight; $A B$ rounded with medium radius of curvature, maximum convexity located below mid H; AVB short and slightly convex; VB straight; $\mathrm{AB}$ and AVB laterally flattened; PVB short; $\mathrm{PB}$ acuminated and located at VB level; greatest $\mathrm{H}$ located anteriorly at one-fourth of length; dorsal outline subfusiform; surface smooth; LV larger and overlap RV all around the carapace;.

\section{REMARKS}

The new species differs from Liuzhinia antalyaensis CrasquinSoleau, 2004 described from the Late Permian-Early Triassic interval of Turkey (Antalya Nappes; Crasquin-Soleau et al. 2004) by having a smaller PB and max $\mathrm{H}$ located in front of mid L. It can be compared with $L$. praeantalyaensis from the latest Permian af Meishan section, Zhejiang Province, South China (Crasquin et al. 2010a) by having AB located below mid $\mathrm{H}$ and the smaller PB but the carapace of $L$. naramase $i$ Chitnarin, n. sp. is thicker in dorsal view.

\section{Genus Baschkirina Rozdestvenskaja, 1959}

TYPE SPECIES. - Baschkirina memorabilis Rozdestvenskaja, 1959 by original designation.

\section{Baschkirina ruchae Chitnarin, n. sp.}

(Figs 24A-D; 25)

TyPE MATERIAL. - Holotype, one complete carapace (SUT-092375, Fig. 24A); paratypes, two complete carapaces (SUT-09-2374, Fig. 24C and SUT-09-2380, Fig. 24D). 
Eтумодоgy. — In honour to Mrs Rucha Ingavat-Helmkce.

MATERIAL EXAMINED. - 17 complete carapaces.

Dimensions. - $\mathrm{H}=0.35-0.51 \mathrm{~mm}, \mathrm{~L}=0.55-0.73 \mathrm{~mm}, \mathrm{H} / \mathrm{L}=0.64-$ 0.69, Holotype: $\mathrm{H}=0.51 \mathrm{~mm}, \mathrm{~L}=0.74 \mathrm{~mm}$; Paratype (Fig. 24C): $\mathrm{H}=0.39 \mathrm{~mm}, \mathrm{~L}=0.59 \mathrm{~mm}$; Paratype (Fig. 24D): $\mathrm{H}=0.53 \mathrm{~mm}$, $\mathrm{L}=0.82 \mathrm{~mm}$ (Fig. 25).

TYPe HORIZON. - Sample 08LO02-2, Nam Maholan section, Nam Maholan Formation, Loei Province, Early Permian.

TyPe LOCALITy. - Sample 08LO02-2, Nam Maholan section (17 $\left.066^{\prime} 23^{\prime \prime} \mathrm{N}, 101^{\circ} 52^{\prime} 48^{\prime \prime} \mathrm{E}\right)$, Nam Maholan Formation, Loei Province, Early Permian.

OCCURRENCES. - Sample 08LO02-2, Nam Maholan section, Nam Maholan Formation, Loei Province, northeast Thailand, Early Permian; sample 07PB04-5, Nong Phai section, Pha Nok Khao Formation, Phetchabun Province, central Thailand, Early Permian.

DiAGNOSIS. - Species of Baschkirina with subtriangular carapace, highly arched dorsal outline, and $\mathrm{AB}$ laterally compressed with maximum convexity located below mid $\mathrm{H}$, flanks subparallel in dorsal view.

\section{DESCRIPTION}

Carapace subtriangular; dorsal outline highly arched; DB straight and inclined at $30^{\circ}$ rearward; $\mathrm{ADB}$ long, straight to slightly convex, with an angle of $120^{\circ}$ with $\mathrm{DB}$; PDB inclined sharply, making angle of $150^{\circ}$ with $\mathrm{DB}$; $\mathrm{AB}$ rounded with medium radius of curvature, maximum convexity located below mid H, and flattened laterally; AVB short; VB straight; PVB short; PB small, sub-spinose and located at or just above ventral level; LV slightly overlaps RV all around the carapace; greatest $\mathrm{H}$ located at mid L; in dorsal view, carapace thick, flanks subparallel and AB laterally compressed.

\section{REMARKS}

Baschkirina ruchae Chitnarin, n. sp. can be compared with B. buekkensis Kozur, 1985 from the Pennsylvanian of French Pyrénées and Bükk Mts, Hungary (Kozur 1985b: 90-91, pl. 22, fig. 1); however, B. buekkensis is characterized by a smaller $\mathrm{H}$, a longer DB and the position of maximum $\mathrm{H}$ in front of mid L. It can be differentiated from B. shii Kozur, 1985 from the Wuchiapingian of Bükk Mts, Hungary (Kozur 1985a: 92, pl. 22, fig. 4) by an $A B$ with smaller radius of curvature and strongly compressed laterally.

\section{Baschkirina cf. ballei Crasquin, 2010}

(Fig. 24I-L)

Baschkirina ballei Crasquin in Crasquin, Forel, Feng, Yuan, Baudin \& Collin, 2010a: 358, fig. 23R-W.

MATERIAl EXAMINED. - 35 complete carapaces.

Dimensions. $-\mathrm{H}=0.23-0.42 \mathrm{~mm}, \mathrm{~L}=0.32-0.64 \mathrm{~mm}, \mathrm{H} / \mathrm{L}=$ $0.65-0.75$.

OCCURRENCES. - Samples 08LO02-1, 08LO02-2 and 08LO02-10, Tham Nam Maholan section, Nam Maholan Formation, Loei Province, northeastern Thailand, Early Permian; sample 07LB04-8, Phu

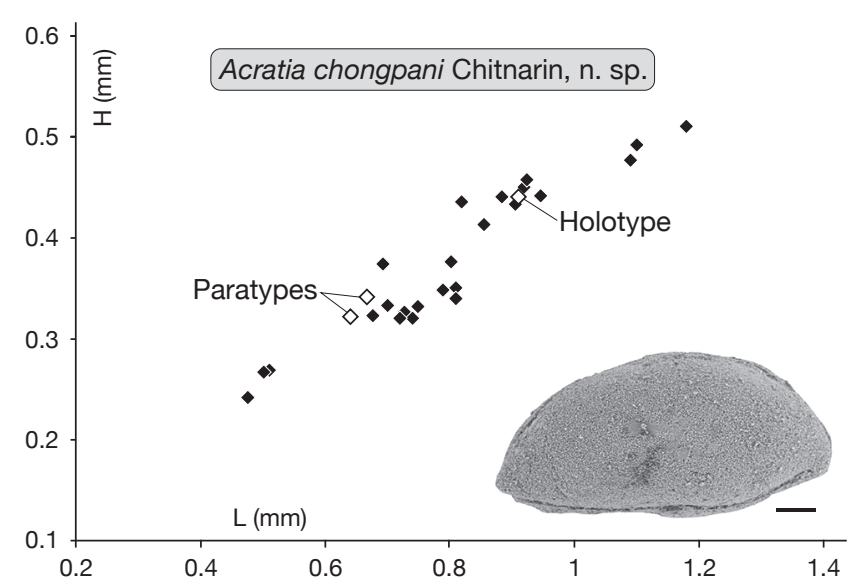

FIG. 22. - Height (H)-length (L) diagram of Acratia chongpani Chitnarin, n. sp. Scale bar: $100 \mu \mathrm{m}$.

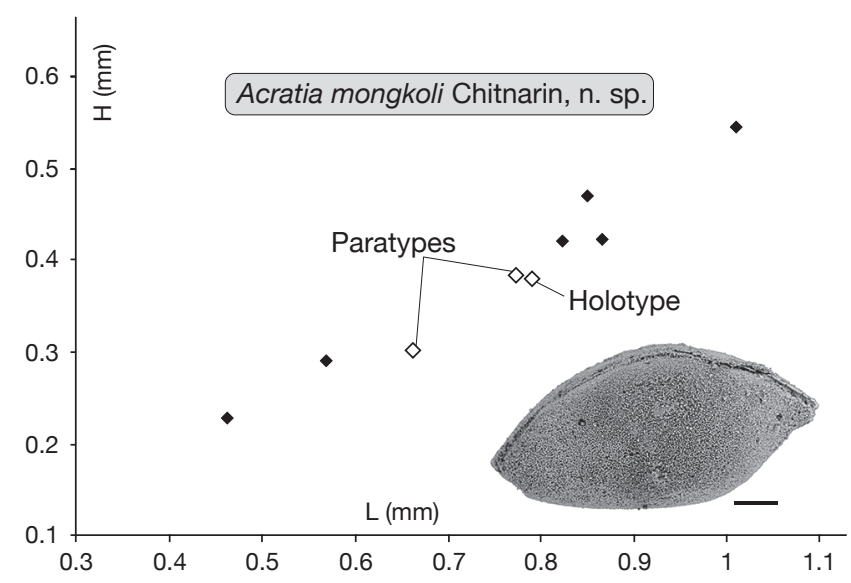

FIG. 23. - Height (H)-length (L) diagram of Acratia mongkoli Chitnarin, n. sp. Scale bar: $100 \mu \mathrm{m}$.

Lam Yai section, Tak Fa Formation, Nakhon Sawan Province, central Thailand, Early Permian; sample 07PB03-3, Khao Kana section, Pha Nok Khao Formation, Phetchabun Province, central Thailand, Early Permian; sample 07PB04-5, Nong Phai section, Pha Nok Khao Formation, Phetchabun Province, central Thailand, Early Permian.

\section{REMARKS}

The studied specimens look similar to Baschkirina ballei Crasquin, 2010 from latest Permian of Meishan section, South China (Crasquin et al. 2010a: 358, figs 23R-W). Baschkirina ballei have longer PDB and less compressed $\mathrm{AB}$.

Baschkirina cf. huzhouensis Forel, 2010

(Fig. 21N-P)

Baschkirina huzhouensis Forel in Crasquin, Forel, Feng, Yuan, Baudin \& Collin, 2010a: 359, fig. 23E-H.

MATERIAL EXAMINED. - Five complete carapaces.

OCCurrences. - Sample 08LB01-1, Khao Som Phot section, Tak Fa Formation, Lopburi Province, central Thailand, Middle Permian.

DiMENSIONS. - $\mathrm{H}=0.23-0.26 \mathrm{~mm}, \mathrm{~L}=0.45-0.49 \mathrm{~mm}, \mathrm{H} / \mathrm{L}=0.50-0.52$. 


\section{REMARKS}

Our specimens are similar to B. huzhouensis Forel, 2010 from the latest Permian of Meishan section, South China (Crasquin et al. 2010a: 359, fig. 23E-H) in having long carapace with straight $\mathrm{DB}$ and $\mathrm{PDB}$. However, $\mathrm{AB}$ of $\mathrm{B}$. cf. huzhouensis is less compressed and located above mid $\mathrm{H}$, but $\mathrm{AB}$ is located at mid $\mathrm{H}$ in our specimens.

\section{Genus Bohlenatia Gründel, 1962}

TyPe SPECIEs. - Acratia inornata Cordell, 1952 by subsequent designation of Gründel (1962: 88).

\section{Bohlenatia sp.}

(Fig. 13J)

MATerial EXAMined. - One complete carapace.

OCCURRENCES. - Sample 08LO02-1, Tham Nam Maholan section, Nam Maholan Formation, Loei Province, northeast Thailand, Early Permian.

Dimensions. $-\mathrm{H}=0.268 \mathrm{~mm}, \mathrm{~L}=0.586 \mathrm{~mm}, \mathrm{H} / \mathrm{L}=0.49$.

\section{REMARKS}

The studied specimen has an $\mathrm{AB}$ with very small radius of curvature and pointing upward, straight VB. It resembles Bohlenatia bukkensis Kozur, 1985 (Kozur 1985a: 86, pl. 18, fig. 7) in lateral outline. However here, the posterior dorsal point is located more ventrally than in B. bukkensis and the PDB is steeper than in B. bukkensis. Note here PDB and AVB are parallel.

Suborder SIGILLIOCOPINA Martens, 1992

Superfamily SigiLlioidea Mandelstam, 1960

Family MicrocheILINELLIDAE Gram, 1975

Genus Microcheilinella Geis, 1933

TYPE SPECIES. - Microcheilus distortus Geis, 1932 by original designation.

Microcheilinella shicheni Crasquin, 2010

(Fig. 26R-S)

Microcheilinella shicheni Crasquin in Crasquin, Forel, Feng, Yuan, Baudin \& Collin, 2010a: 31, fig. 22H, K, L, Q.

Microcheilinella subreniformis Chen, 1958: 253, pl. 2, figs 7-11, 24-29. - Chen \& Shi 1982: 141, pl. 12, figs 1-6. — Shi \& Chen 1987; 61, pl. 14, fig. 14-22; 2002: 93, pl. 25, figs 15-18.

MATERIAL EXAMINED. - One complete carapace and one broken carapace.

OCCURRENCES. - Late Permian of Guangxi Province (Shi \& Chen 2002), of Meishan section, Zhejian Province (Shi \& Chen 1987 and Crasquin et al. 2010a); Sample 07PB08-2, Ban Naen Sawan II section, Pha Nok Khao Formation, Phetchabun Province, central Thailand, Middle Permian.

Dimensions. $-\mathrm{H}=0.24 \mathrm{~mm}, \mathrm{~L}=0.56 \mathrm{~mm}, \mathrm{H} / \mathrm{L}=0.42$
Suborder CYTHEROCOPINA Baird, 1850

Superfamily CYTHEROIDAE Baird, 1850

Family CYTHERIDEIDAE Sars, 1925

Genus Basslerella Kellett, 1935

TyPE SPECIES. - Basslerella crassa Kellett, 1935 by original designation.

Basslerella wipanuae Chitnarin, n. sp.

(Figs 27A-E; 28)

TYPE MATERIAL. - Holotype, complete carapace (SUT-09-2429, Fig. 27A); paratypes, two complete carapaces (SUT-09-2430; Fig. 27B and SUT-09-2421; Fig. 27D)

ETYMology. - In honour of Dr Wipanu Rukmai, Northeastern Research Institute of Petrified Wood and Mineral Resources, Nakhon Ratchasima Rajabhat University, Nakhon Ratchasima, Thailand.

MATERIAL EXAMINED. - 60 complete carapaces.

DiMENSIONS. $-\mathrm{H}=0.28-0.36 \mathrm{~mm}, \mathrm{~L}=0.36-0.48 \mathrm{~mm}, \mathrm{H} / \mathrm{L}=0.70$ 0.80, Holotype: $\mathrm{H}=0.31 \mathrm{~mm}, \mathrm{~L}=0.44 \mathrm{~mm}$; Paratype (Fig. 27B): $\mathrm{H}=0.35, \mathrm{~L}=0.48 \mathrm{~mm}$; Paratype (Fig. 27D): $\mathrm{H}=0.34 \mathrm{~mm}, \mathrm{~L}=$ $0.43 \mathrm{~mm}$ (Fig. 28).

Type HORIZON. - Sample 07PB03-5, Khao Kana section, Pha Nok Khao Formation, Sakmarian, Early Permian.

TyPE LOCALITY. - Sample number 07PB03-5, Khao Kana section (160.'12"N, 10054’20"E), Pha Nok Khao Formation, Phetchabun province, central Thailand.

OCCURRENCES. - Sample 08LO07-10, Sak Chai Quarry section, Pha Nok Khao Formation, Chaiyaphum Province, northeastern Thailand, Early Permian; samples 07PB03-1, 07PB03-5, Khao Kana section, Pha Nok Khao Formation, Phetchabun Province, central Thailand, Early Permian; samples 07PB04-2, 07PB04-5, 07PB04-7, Nong Phai section, Pha Nok Khao Formation, Phetchabun Province, central Thailand, Early Permian; sample 07PB05-5, Ban Naen Sawan I section, Tak Fa Formation, Phetchabun Province, central Thailand, Middle Permian; sample 07LB04-8, Phu Lam Yai section, Tak Fa Formation, Nakhon Sawan province, central Thailand, Early Permian; sample 07LB05-5, Ta Kli section, Tak Fa Formation, Nakhon Sawan province, central Thailand, Early Permian; samples 07LB09-1, 07LB09-2, Khao Phu Chong Kho locality, Tak Fa Formation, Nakhon Sawan province, central Thailand, Early Permian.

DiAgNosis. - Species of Basslerella with triangular carapace, AB and $\mathrm{PB}$ round with nearly the same small radius of curvature, $\mathrm{H}$ max at mid L, faint striation with digital print aspect oriented from $\mathrm{AD}$ to PV on carapace surface.

\section{DESCRIPTION}

Carapace small, subtriagular; dorsal outline highly arched; $\mathrm{DB}$ short; $\mathrm{ADB}$ convex, forming in angle of $130^{\circ}$ with $\mathrm{DB}$; $\mathrm{AB}$ rounded with large radius of curvature, maximum convexity located at lower third of $\mathrm{H}$; AVB short and convex; VB slightly convex; ventral laterally inflated; PVB short and convex; PB rounded with smaller radius of curvature, maximum convexity located ventrally, at lower fourth of $\mathrm{H}$; PDB short; greatest $\mathrm{H}$ located at mid L; faint striation with digital print aspect developed on the surface oriented from AD to 

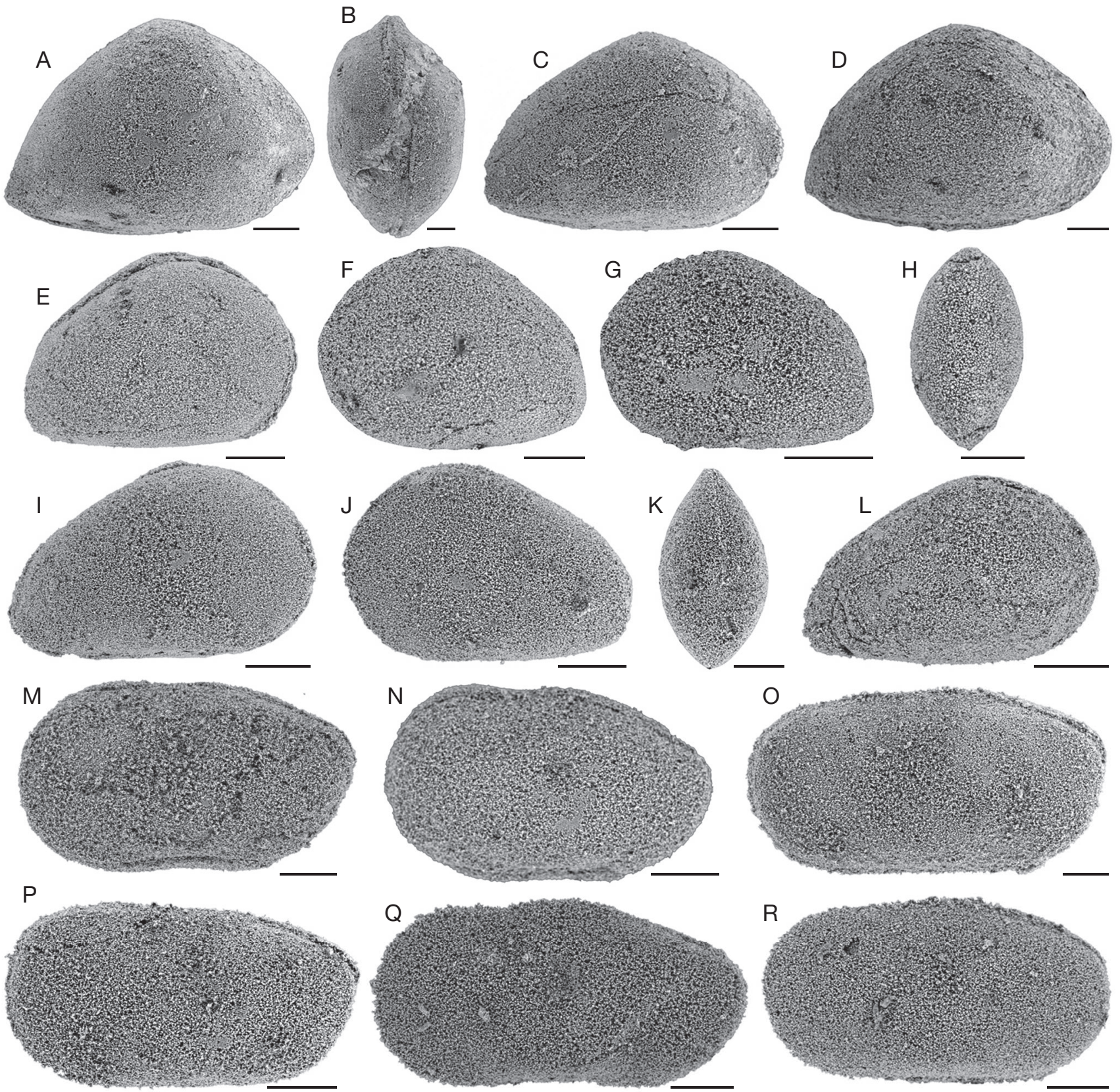

FIG. 24. - Ostracods from the limestones of the Indochina Block, Central Thailand. All specimens are stored at the Suranaree University of Technology Collections (Nakhon Ratchasima, Thailand) numbers SUT-09-xxxx. All the specimens are represented by complete carapaces. A-D, Baschkirina ruchae Chitnarin, n. sp.;

A, holotype, right lateral view, SUT-09-2375, sample 08LO02-2; B, dorsal view, SUT-09-2376, sample 08LO02-2; C, paratype, right lateral view, SUT-09-2374, sample 08LO02-2; D, paratype, right lateral view, SUT-09-2380, sample 08LO02-5; E-H, Basslerella tota Chen and Bao, 1986; E, right lateral view, SUT-09-2389, sample 07PB04-5; F, left lateral view, SUT-09-2387, sample 07LB04-8; G, left lateral view, SUT-09-2383, sample 07LB04-8; H, dorsal view, SUT-09-2385, sample 07LB04-8; I-L, Baschkirina cf. ballei Crasquin, 2010; I, right lateral view, SUT-09-2410, sample 08LO02-2; J, left lateral view, SUT-09-2414, sample 08LO02-2; K, dorsal view, SUT-09-2412, sample 08LO02-2; L, right lateral view, SUT-09-2405, sample 07PB04-5; M, N, Sulcella suprapermiana Kozur, 1985; M, right lateral view, SUT-09-2662, sample 07PB03-1; N, right lateral view, SUT-09-2664, sample 07PB03-3; O-R, Sulcella mesopermiana Kozur, 1985; O, right lateral view, SUT-09-2665, sample 08LB01-1; P, right lateral view, SUT-09-2666, sample 08LB01-1; Q, right lateral view, SUT-09-2676, sample 07PB03-1; R, right lateral view, SUT-09-2682, sample 07PB03-1. Scale bars: $100 \mu \mathrm{m}$.

PV; in dorsal view, carapace biconvex with maximum thickness at mid lenght; LV larger and slightly overlaping RV all around the carapace.

\section{REMARKS}

Basslerella wipanuae Chitnarin, n. sp. is characterized by its diagnosis characters. The ornamentation is different from the reticulation of B. reticulata Shi, 1987 from the latest Permian of Meishan section, Zhejiang Province, East China (Shi \& Chen 1987: 55, pl. 17, figs 7-12). The more elliptical form of $B$. wipanuae is similar to $B$. annesophieae Crasquin, 2010 (Crasquin et al. 2010a: 362-363, fig. $20 \mathrm{~N}-\mathrm{V}$ ), but PDB of $B$. wipanuae is more rounded than in $B$. annesophieae. 


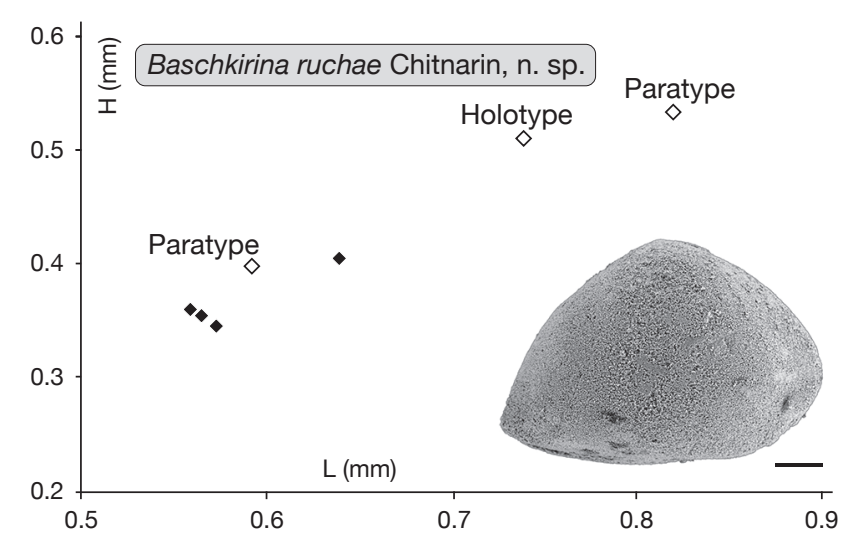

FIG. 25. - Height (H)-length (L) diagram of Baschkirina ruchae Chitnarin, n. sp. Scale bar: $100 \mu \mathrm{m}$.

\section{Basslerella naresi Chitnarin, n. sp.}

(Figs 27F-J; 29)

Type MATERIAL. - Holotype, one complete carapace (SUT-092463, Fig. 27G); paratypes, two complete carapaces (SUT-09-2486, Fig. 27F and SUT-09-2477, Fig. 27J).

ETYMOLOGY. - In honour of Mr Nares Sattayalak, PTTEP, Thailand.

MATERIAl EXAMINED. - 21 complete carapaces.

Dimensions. $-\mathrm{H}=0.20-0.56 \mathrm{~mm}, \mathrm{~L}=0.30-0.83 \mathrm{~mm}, \mathrm{H} / \mathrm{L}=0.64$ 0.74, Holotype: $\mathrm{H}=0.50 \mathrm{~mm}, \mathrm{~L}=0.77 \mathrm{~mm}$; Paratype (Fig. 27F): $\mathrm{H}=0.40, \mathrm{~L}=0.61 \mathrm{~mm}$; Paratype (Fig. 27J): $\mathrm{H}=0.42 \mathrm{~mm}, \mathrm{~L}=$ $0.67 \mathrm{~mm}$ (Fig. 29).

Type Horizon. - Sample number 08LO02-2, Tham Nam Maholan section, Nam Maholan Formation, Asselian, Early Permian.

Type LOCALiTy. - Sample 08LO02-2, Tham Nam Maholan section ( $\left.17^{\circ} 06^{\prime} 23^{\prime \prime} \mathrm{N}, 101^{\circ} 52^{\prime} 48^{\prime \prime} \mathrm{E}\right)$, Nam Maholan Formation, Loei province, northeastern Thailand.

OCCurREnCES. - Samples 08LO02-1, 08LO02-2, Tham Nam Maholan section, Nam Maholan Formation, Loei province, northeastern Thailand, Early Permian; samples 07LB05-B1, 07LB05-B2, 07LB05-A2, 07LB05-5, Ta Kli section, Tak Fa Formation, Nakhon Sawan province, central Thailand, Early Permian.

DiAgNosis. - Species of Basslerella with long carapace, straight and long $\mathrm{DB}, \mathrm{AB}$ and $\mathrm{PB}$ maximum of curvature located below mid $\mathrm{H}$, and very wide and flat ventral area, dorsal view with water drop aspect.

\section{DESCRIPTION}

Carapace long, subelliptical; dorsal outline broadly arched; $\mathrm{DB}$ straight, inclined at $30^{\circ}$ rearward; $\mathrm{ADB}$ slightly convex; $\mathrm{PDB}$ short, forming an angle of $135^{\circ}$ with $\mathrm{DB} ; \mathrm{AB}$ rounded with large radius of curvature, maximum convexity located, at lower third of $\mathrm{H}$; AVB short and convex; VB straight; ventral area flat; PVB short and convex; PB blunt, located just above $\mathrm{VB}$; greatest $\mathrm{H}$ located at just in front of mid L; surface smooth; LV larger and slightly overlaps RV all around the carapace; carapace with water drop aspect in dorsal view, maximum thickness in the posterior part.

\section{REMARKS}

Basslerella naresi Chitnarin, n. sp. can be recognized very easily by its very wide and flat ventral area. Larger specimens have the wider and thicker posterior part and $\mathrm{ADB}$ rounded. B. naresi Chitnarin, n. sp. can be differentiated from B. annesophieae from the latest Permian of Meishan section, Eastern China (Crasquin et al. 2010a: 362, 363, figs $20 \mathrm{~N}-\mathrm{V}$ ) by the smaller radius of curvature of $\mathrm{AB}$ and the longer carapace.

\section{Basslerella tota Chen \& Bao, 1986} (Fig. 24E-H)

Basslerella tota Chen \& Bao, 1986: 123, pl. 1, figs 31, 32, pl. 4 figs 7 , 8. - Crasquin-Soleau et al. 2004: 288, pl. 4, figs 9, 10. - Yi 2004: pl. 2, fig. 20. - Crasquin et al. 2008b: pl. 5, figs 17, 18. — Forel 2012: 22, fig. 14O-Q.

MATERIAL EXAMINED. - 35 complete carapaces.

DimENSIONS. $-\mathrm{H}=0.23-0.42 \mathrm{~mm}, \mathrm{~L}=0.32-0.64 \mathrm{~mm}, \mathrm{H} / \mathrm{L}=0.71-0.74$.

Occurrences. - Jiangsu Province, Early Permian (Chen \& Bao 1986); Hydra Island, Greece, late Middle Permian (Crasquin-Soleau \& Baud 1998); Western Taurus, Turkey, Late Permian (Crasquin-Soleau et al. 2004); Meishan section, East China, latest Permian (Crasquin et al. 2010a); Fujian province, South China, Late Permian (Yi 2004); Dajiang section, Guizhou Province, South China, Late Permian (Forel 2012); samples 08LO02-1, 08LO02-2 and 08LO02-10, Tham Nam Maholan section, Nam Maholan Formation, Loei Province, northeastern Thailand, Early Permian; sample 07LB04-8, Phu Lam Yai section, Tak Fa Formation, Nakhon Sawan Province, central Thailand, Early Permian; sample 07PB03-3, Khao Kana section, Pha Nok Khao Formation, Phetchabun Province, central Thailand, Early Permian; sample 07PB04-5, Nong Phai section, Pha Nok Khao Formation, Phetchabun Province, central Thailand, Early Permian.

Order PLATYCOPIDA Sars, 1866

Suborder PLATYCOPINA Sars, 1866

Superfamily CAVELLINOIDEA Egorov, 1950

Family CAVELlinidaE Egorov, 1950

Genus Cavellina Coryell, 1928

TYPE SPECIES. - Cavellina pulchella Coryell, 1928 by original designation.

\section{Cavellina sp.}

(Fig. 13M-P)

MATERIAL EXAMINED. - 12 complete carapaces.

OCCURRENCES. - Samples 07PB03-1, 07PB03-3, Khao Kana section, Pha Nok Khao Formation, Phetchabun Province, central Thailand, Early Permian; samples 07LB05-A3, 07LB05-A2, Ta Kli section, Tak Fa Formation, Nakhon Sawan province, central Thailand, Early Permian.

Dimensions. $-\mathrm{H}=0.31-0.41 \mathrm{~mm}, \mathrm{~L}=0.49-0.68 \mathrm{~mm}$

\section{REMARKS}

Cavellina sp. has subelliptical carapace, broadly arched dorsal outline, round $\mathrm{AB}$ and $\mathrm{PB}$, and strong overlap of $\mathrm{LV}$ on $\mathrm{RV}$ at $\mathrm{DB}$ and $\mathrm{VB}$. 

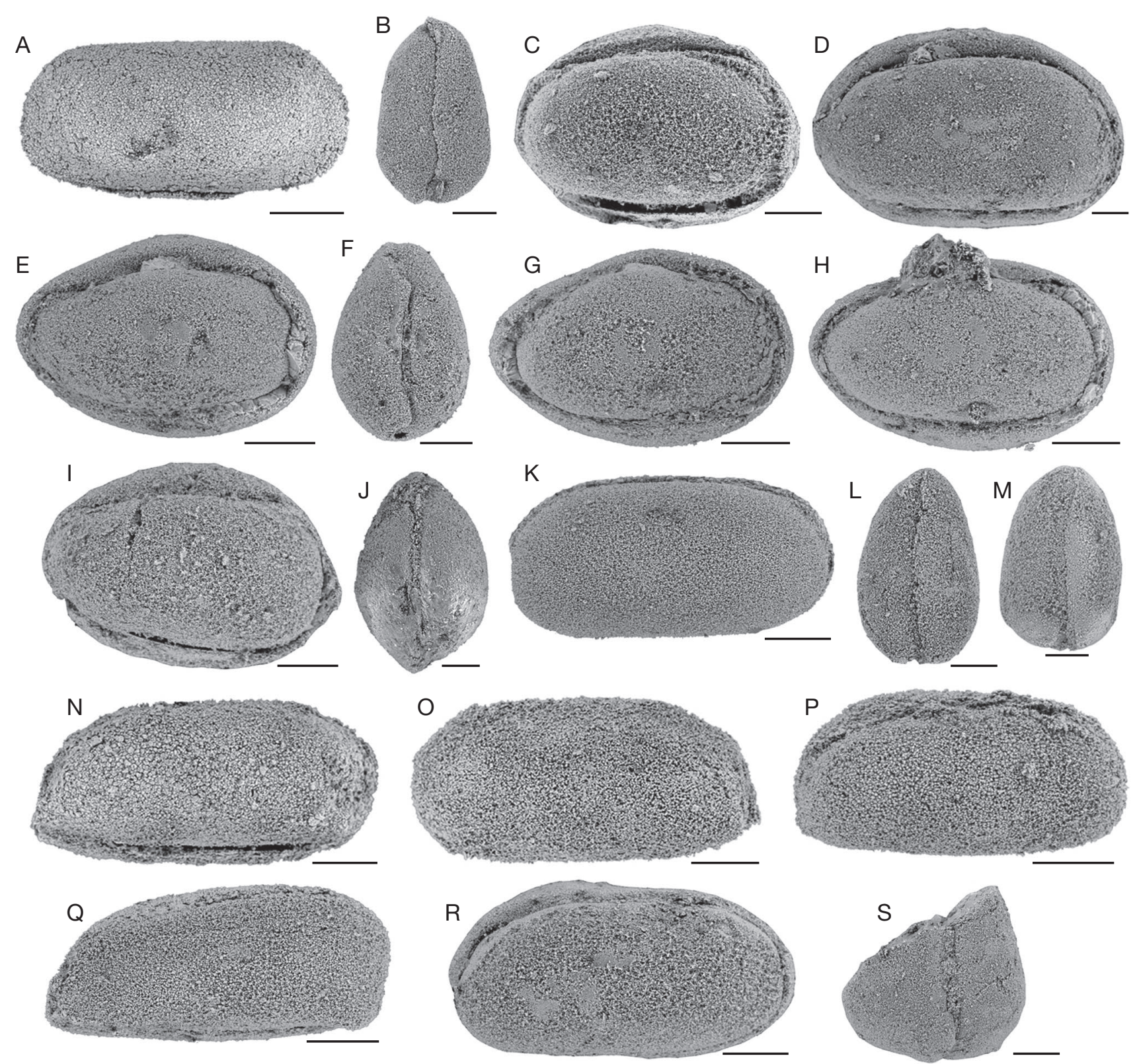

FIG. 26. - Ostracods from the limestones of the Indochina Block, Central Thailand. All specimens are stored at the Suranaree University of Technology Collections (Nakhon Ratchasima, Thailand) numbers SUT-09-xxxx. All the specimens, except $\mathbf{S}$ which is broken, are represented by complete carapaces. A, B, Microcheilinella sp. A; A, right lateral view, SUT-09-2547, sample 07PB04-2; B, ventral view, SUT-09-2548, sample 07PB04-2; C, Microcheilinella sp. B, right lateral view, SUT-09-2549, sample 08LB01-2; D, Microcheilinella sp. C, right lateral view, SUT-09-2550, sample 07PB08-3; E-H, Microcheilinella sp. D; E, right lateral view, SUT-09-2551, sample 07PB05-2; F, dorsal view, SUT-09-2554, sample 07PB05-2; G, right lateral view, SUT-09-2552, sample 07PB05-2; H, right lateral view, SUT-09-2553, sample 07PB05-2; I, J, Microcheilinella sp. E; I, right lateral view, SUT-09-2555, sample 07LB05-B1; J, dorsal view, SUT-09-2556, sample 07LB05B1; K, L, Microcheilinella sp. F; K, right lateral view, SUT-09-2559, sample 07PB04-2; L, ventral view, SUT-09-2560, sample 07PB04-2; M-Q, Microcheilinella sp. G, 2010; M, ventral view, SUT-09-2564, sample 08LO07-1; N, right lateral view, SUT-09-2561, sample 07PB04-2; O, left lateral view, SUT-09-2562, sample 07PB04-2; P, right lateral view, SUT-09-2563, sample 08LO07-1; Q, right lateral view, SUT-09-2565, sample 07PB04-2; R, S, Microcheilinella shicheni Crasquin, 2010; R, right lateral view, SUT-09-2566, sample 08PB02-13; L, ventral view, SUT-09-2567, sample 08PB02-13. Scale bars: 100 4m.

Genus Sulcella Coryell \& Sample, 1932

TYPE SPECIES. — Sulcella sulcata Coryell \& Sample, 1932 by original designation.

\section{Sulcella mesopermiana Kozur, 1985}

(Fig. 24O-R)

Sulcella mesopermiana Kozur, 1985b: 22, pl. 5, figs 3, 4. - Crasquin-Soleau \& Baud 1998: pl. 3, figs 3, 5, 6, 9. - ?Zazzali et al. 2015: 306, fig. 17A.

\section{MATERIAL EXAMINED. — 43 complete carapaces.}

Occurrences. - Bükk Mountains, Hungary, Middle and Late Permian (Kozur 1985b); Hydra Island, Greece, Early to Late Permian (Crasquin-Soleau \& Baud 1998); ?Chaotian section, Sichuan, South China, earliest Late Permian (Zazzali et al. 2015); samples 07PB03-1, 07PB03-3, Khao Kana section, Pha Nok Khao Formation, Phetchabun Province, central Thailand, Early Permian.

Dimensions. $-\mathrm{H}=0.30-0.47 \mathrm{~mm}, \mathrm{~L}=0.51-0.64 \mathrm{~mm}$. 

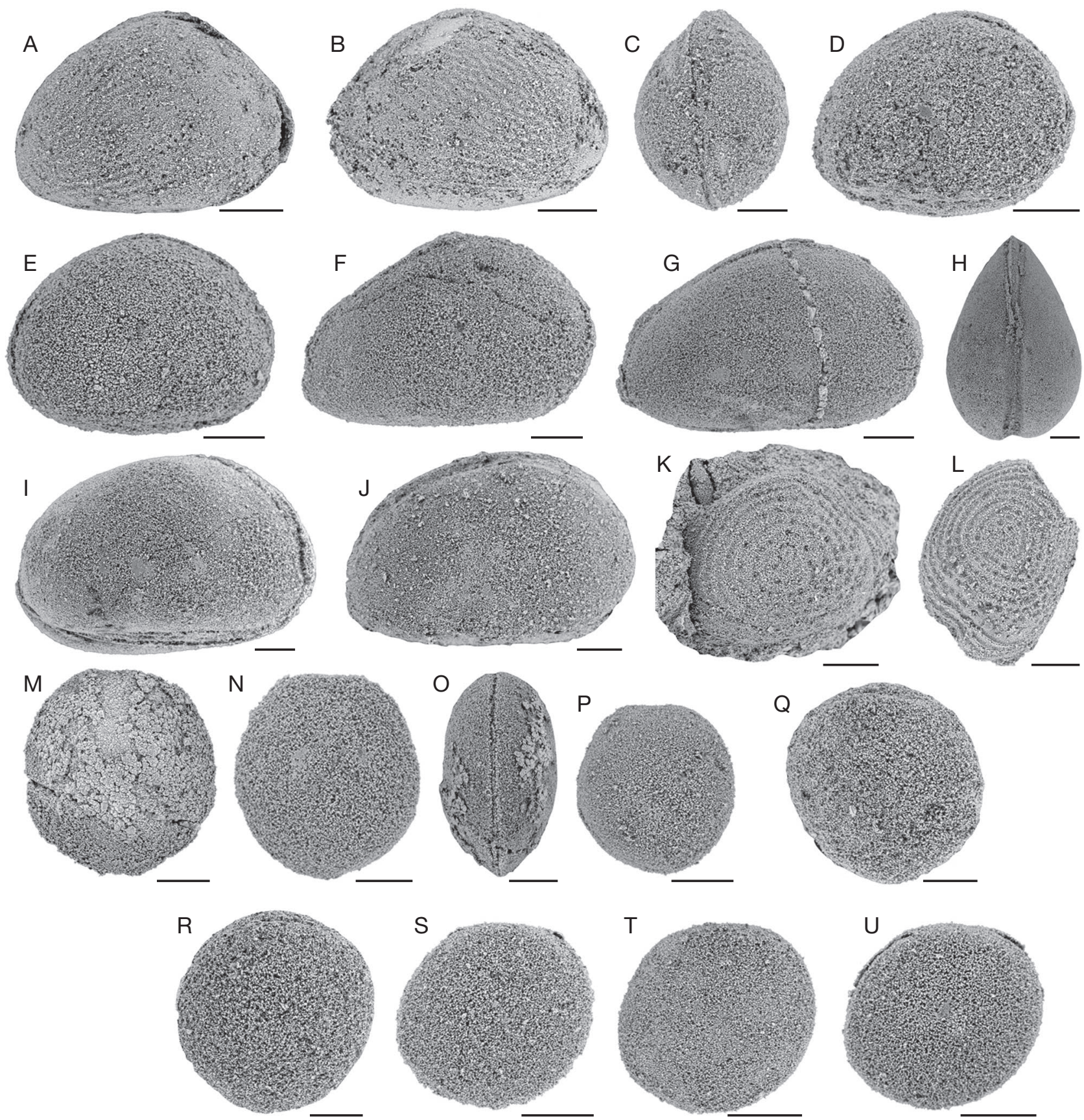

FIG. 27. - Ostracods from the limestones of the Indochina Block, Central Thailand. All specimens are stored at the Suranaree University of Technology Collections (Nakhon Ratchasima, Thailand) numbers SUT-09-xxxx. All the specimens, except K and L, are represented by complete carapaces. A-E, Basslerella wipanuae Chitnarin, n. sp; A, holotype, right lateral view, SUT-09-2429, sample 07PB03-5; B, paratype, left lateral view, SUT-09-2430, sample 07PB03-5; C, ventral view, SUT-09-2426, sample 07PB04-2; D, paratype, right lateral view, SUT-09-2421, sample 07PB03-5; E, right lateral view, SUT-09-2425, sample 07PB04-2. F-J, Basslerella naresi Chitnarin, n. sp.; F, left lateral view, SUT-09-2486, sample 08LO02-1; G, holotype, left lateral view, SUT-09-2463, sample 08LO02-2; H, ventral view, SUT-09-2462, sample 08LO02-2; I, left lateral view, SUT-09-2461, sample 08LO02-2; J, left lateral view, SUT-09-2477, sample 07LB05-A2. K, L, Polycope sp.; K, SUT-09-2604, sample 07PB06-5; L, SUT-09-2606, sample 07PB06-5. M-P, Polycope sp.A; M, left lateral view, SUT-09-2610, sample 07PB04-2; N, left lateral view, SUT-09-2621, sample 08LO02-2; O, anterior view, SUT-09-2615, sample 07PB04-2; P, left lateral view, SUT-09-2627, sample 07LB05-B2. Q, R, Polycope sp. B; Q, left lateral view, SUT-09-2628, sample 08LO02-5; R, left lateral view, SUT-09-2630, sample 08LO02-5. S-U, Polycope sp.C; S, left lateral view, SUT-09-2644, sample 07LB09-1; T, left lateral view, SUT-09-2643, sample 07LB09-1; U, left lateral view, SUT-09-2642, sample 07LB09-1. Scale bars: 100 4 m.

\section{Sulcella suprapermiana Kozur, 1985}

(Fig. 24M, N)

Sulcella suprapermiana Kozur, 1985b: 22, pl. 5, figs 3-4. - Crasquin-Soleau \& Baud 1998: pl.4, figs 1-3. - Crasquin-Soleau et al. 1999: pl. 4, fig. 15. - Crasquin et al. 2008a: 255, pl. 6, fig. 1-2. Zazzali et al. 2015: 306, fig. 17B, C.
MATERIAL EXAMINED. - Six complete carapaces.

Occurrences. - Bűkk Mountains, Hungary, Middle and Late Permian (Kozur 1985b); Hydra Island, Greece, Early to Late Permian (Crasquin-Soleau \& Baud 1998); Khuff Formation, Sultanate of Oman, Middle Permian (Crasquin-Soleau et al. 1999); Bulla section, northern Italy, Late Permian (Crasquin et al. 2008a); 
Chaotian section, Sichuan, South China, earliest Late Permian (Zazzali et al. 2015); samples 07PB03-1, 07PB03-3, 07PB035, Khao Kana section, Pha Nok Khao Formation, Phetchabun Province, central Thailand, Early Permian; samples 07LB04-8, 07LB04-12, 07LB04-13, 07LB04-17, Phu Lam Yai section, Tak Fa Formation, Nakhon Sawan province, central Thailand, late Early Permian; sample 07LB05-C3, Ta Kli section, Tak Fa Formation, Nakhon Sawan province, central Thailand, Early Permian; sample 08LB01-1, Khao Som Phot section, Tak Fa Formation, Lopburi Province, central Thailand, Middle Permian.

Dimensions. $-\mathrm{H}=0.27-0.50 \mathrm{~mm}, \mathrm{~L}=0.48-0.89 \mathrm{~mm}, \mathrm{H} / \mathrm{L}=$ $0.50-0.56$

Subclass MYODOCOPA Sars, 1866

Order HALOCYPRIDA Dana, 1853

Suborder CLADOCOPINA Sars, 1866

Family POLYCOPIDAE Sars, 1866

Genus Polycope Sars, 1866

TYPE SPECIES. - Polycope orbicularis Sars, 1866 by original designation.

\section{Polycope sp. A}

(Fig. 27M-P)

MATERIAL EXAMINED. - Seven complete carapaces.

OCCURRENCES. - Samples 08LO02-1, 08LO02-2, Tham Nam Maholan section, Nam Maholan Formation, Loei province, northeastern Thailand, Early Permian; sample 07PB04-2, Nong Phai section, Pha Nok Khao Formation, Phetchabun Province, central Thailand, Early Permian; sample 07LB05-B2, Ta Kli section, Tak Fa Formation, Nakhon Sawan province, central Thailand, Early Permian; sample 08LB01-1, Khao Som Phot section, Tak Fa Formation, Lopburi Province, central Thailand, Middle Permian.

Dimensions. $-\mathrm{H}=0.28-0.45 \mathrm{~mm}, \mathrm{~L}=0.26-0.40 \mathrm{~mm}, \mathrm{H} / \mathrm{L}=$ 1.07-1.6.

\section{REMARKS}

Polycope sp. A is characterized by high and suboval carapace with straight $\mathrm{DB}$.

\section{Polycope sp. B}

(Fig. 27Q-R)

MATERIAL EXAMINED. - Three complete carapaces.

OCCURRENCES. - Sample 08LO02-5, Tham Nam Maholan section, Nam Maholan Formation, Loei province, northeastern Thailand, Early Permian.

Dimension. $-\mathrm{H}=0.39-0.40 \mathrm{~mm}, \mathrm{~L}=0.39-0.40 \mathrm{~mm}, \mathrm{H} / \mathrm{L}=1.00$

\section{REMARKS}

Polycope sp. B is subequivalved and has subcircular carapace. $\mathrm{DB}$ is convex on both valves with an incised dorsum and $\mathrm{H} / \mathrm{L}$ ratio is of 1.00 .

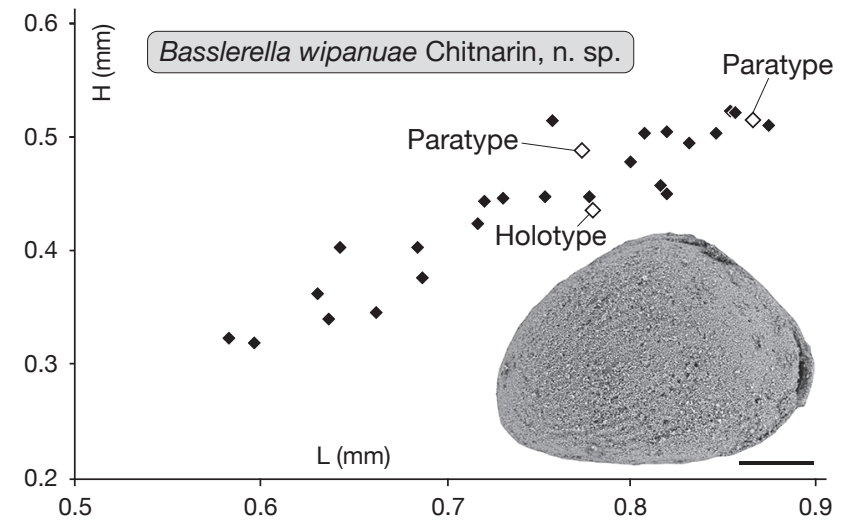

FIG. 28. - Height (H)-length (L) diagram of Basslerella wipanuae Chitnarin n. sp. Scale bar: $100 \mu \mathrm{m}$

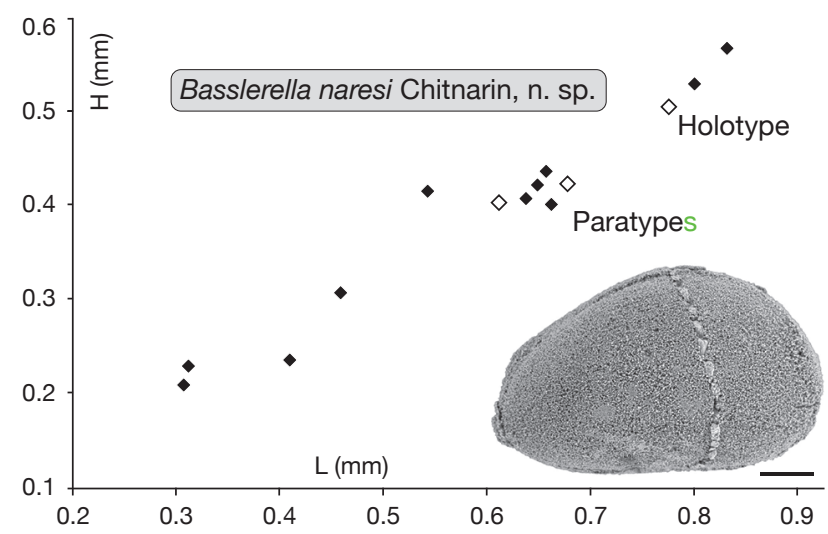

FIG. 29. - Height (H)-length (L) diagram of Basslerella naresi Chitnarin, n. sp. Scale bar: $100 \mu \mathrm{m}$.

\section{Polycope sp. C}

(Fig. 27S-U)

MATERIAL EXAMINED. - Six complete carapaces.

OCCuRRENCES. - Samples 08LO02-6, 08LO02-10 Tham Nam Maholan section, Nam Maholan Formation, Loei province, northeastern Thailand, Early Permian; samples 07PB04-2, 07PB04-5, Nong Phai section, Pha Nok Khao Formation, Phetchabun Province, central Thailand, Early Permian; sample 07LB09-1, Khao Phu Chongkho locality, Tak Fa Formation, Nakhon Sawan province, central Thailand, late Early Permian.

DimensiONS. $-\mathrm{H}=0.25-0.48 \mathrm{~mm}, \mathrm{~L}=0.27-0.54 \mathrm{~mm}, \mathrm{H} / \mathrm{L}=$ 0.88-0.96

\section{REMARKS}

Polycope sp. C has subcircular carapace and straight DB. Polycope sp. C differs from $P$. sp. A by the less $\mathrm{H} / \mathrm{L}$ ratio, and from $P$. sp. B by straight DB and absence of the incised dorsum.

\section{Polycope sp. D}

(Fig. 27K-L)

MATERIAL EXAMINED. - Three incomplete carapaces. 
TABLE 3. - Relationships between the Early to Middle Permian ostracods from Indochina block of central Thailand and other known Permian localities. Column abbreviations: 1, Bradfield 1935, Cooper 1946, Cordell 1952, Western USA, upper Carboniferous; 2, Knight 1928, Missouri, USA, upper Carboniferous; 3, Kellett 1934, Kansas, USA, upper Carboniferous to lower Permian; 4, Chen 1958, Nanking, Eastern China, lower Permian; 5, Guan et al. 1978, Hunan, South China, lower Permian; 6, Chen \& Bao 1986, Jiangsu, Eastern China, lower Permian; 7, Kozur 1985a, Bükk Mts, Hungary, upper Permian; 8, Crasquin et al.1999, Sultanate Oman, middle Permian; 9, Crasquin-Soleau \& Baud 1998, Hydra Island, Greece, upper Permian; 10, Ishizaki 1964, Miyagi, Northeast Japan, middle Permian; 11, Lethiers et al. 1989, Jebel Tebaga, Tunisia, middle Pemian; 12, Chen \& Shi 1982, Jiangsu, China, upper Permian; 13, Shi \& Chen 1987, Zhejiang, Eastern China, upper Permian; 14, Shi \& Chen 2002, Guanxi, South China, upper Permian; 15, Crasquin-Soleau et al. 2007, Tibet, China, upper Permian; 16, Crasquin et al. 2008b, Alps, Italy, Permian-Triassic; 17, Crasquin et al. 2010b, Vardar, NW Serbia, upper Permian; 18, Crasquin et al. 2010a, Zhejiang, Eastern China, upper Permian; 19, Forel 2012, Guizhou, South China, upper Permian; 20, Zazzali et al. 2015, Sichuan, South China, upper Permian. Other abbreviations: SI, Simpson Index; na, data not available.

\begin{tabular}{|c|c|c|c|c|c|c|c|c|c|c|c|c|c|c|c|c|c|c|c|c|c|}
\hline $\begin{array}{l}\text { Age of the } \\
\text { studied } \\
\text { sections }\end{array}$ & $\begin{array}{l}\text { Palaeobigeographic relationships of } \\
\text { Early and Middle Permian ostracods from } \\
\text { Indochina block }\end{array}$ & 1 & 2 & 3 & 4 & 5 & 6 & 7 & 8 & 9 & 10 & 11 & 12 & 13 & 14 & 15 & 16 & 17 & 18 & 19 & 20 \\
\hline middle & Microcheilinella shicheni Crasquin, 2010 & - & - & - & - & - & - & - & - & - & - & - & - & - & - & - & - & - & - & - & - \\
\hline Permian & Bairdia guangxiensis Guan, 1978 & - & - & - & - & - & - & - & - & - & - & - & - & $\mathbf{n}$ & - & - & - & - & - & - & - \\
\hline & Bairdiacypris deloi Bradfield, 1935 & - & - & - & - & - & - & - & - & - & - & - & - & - & - & - & - & - & - & - & - \\
\hline lower & Samarella viscusforma Chitnarin, 2012 & - & - & - & - & - & - & - & - & - & - & - & - & - & - & - & - & - & - & - & - \\
\hline Permian & Liuzhinia praeantalayaensis Forel, 2010 & - & - & - & - & - & - & - & - & - & - & - & - & - & - & - & - & - & - & - & - \\
\hline & Bairdia pierrevalentini Crasquin, 2010 & - & - & - & - & - & - & - & - & - & - & - & - & - & - & - & - & - & - & - & - \\
\hline & Bairdia deweveri Crasquin, 2010 & - & - & - & - & - & - & - & - & - & - & - & - & - & - & - & घ & घ & 曰 & - & - \\
\hline & Hollinella $(H$.$) martensiformis Crasquin, 2010$ & - & - & - & - & - & - & - & - & - & - & - & - & - & - & - & - & - & - & - & - \\
\hline & Cyathus elliptica Shi, 1987 & - & - & - & - & - & - & - & - & - & - & - & - & - & - & - & - & - & - & - & - \\
\hline & Bairdia broutini Crasquin, 2010 & - & - & - & - & - & - & - & - & - & - & - & - & - & - & - & - & - & घ & - & - \\
\hline & Bairdia urodeloformis Chen, 1987 & - & - & - & - & - & - & - & - & - & - & - & - & - & ш & - & - & - & - & - & - \\
\hline & Paraparchites chenshii Crasquin, 2010 & - & - & - & - & - & - & - & - & - & - & - & - & - & - & - & - & - & - & - & - \\
\hline & Petasobairdia subnantongensis Chen, 1987 & - & - & - & - & - & - & - & - & - & - & - & - & - & - & - & - & - & - & - & - \\
\hline & Acratia chongpani Chitnarin, n. sp & - & - & - & - & - & - & - & - & - & - & - & - & - & - & - & - & - & - & - & - \\
\hline & Acratia mongkoli Chitnarin, n. sp. & - & - & - & - & - & - & - & - & - & - & - & - & - & - & - & - & - & - & - & - \\
\hline & Hollinella (H.) herrickana (Girty, 1909) & - & - & - & - & - & - & - & 曰 & - & - & 曰 & - & - & - & - & - & - & - & - & - \\
\hline & Sulcella mesopermiana Kozur, 1985 & - & - & - & - & - & - & - & - & - & - & - & - & - & - & - & - & - & - & - & - \\
\hline & Sulcella suprapermiana Kozur, 1985 & - & - & - & - & - & - & $\mathbf{- 1}$ & - & 曰 & - & - & - & - & - & - & - & - & - & - & - \\
\hline & $\begin{array}{l}\text { Bairdia deducta deducta Zalanyi, } 1974 \\
\text { sensu Kozur (1985a) }\end{array}$ & - & - & - & - & - & - & - & - & - & - & - & - & - & - & - & - & - & - & - & - \\
\hline & Cyathus caperata (Guan, 1978) & - & - & - & - & - & - & - & - & - & - & - & - & - & - & - & - & - & - & - & - \\
\hline & $\begin{array}{l}\text { Bairdia menardensis Harlton, } 1929 \\
\text { sensu Chen (1958) }\end{array}$ & - & - & - & - & - & - & - & - & - & - & - & - & - & - & - & - & - & - & - & - \\
\hline & Bairdia altiarcus Chen, 1958 & - & - & - & - & - & - & - & - & - & - & - & - & - & - & - & - & - & - & - & - \\
\hline & Lobobairdia ventriconcava (Chen, 1958) & - & - & - & - & - & - & - & - & - & - & - & - & - & घ & - & - & - & - & - & - \\
\hline & $\begin{array}{l}\text { Bairdia beedei Ulrich \& Bassler, } 1906 \\
\text { sensu Chen (1958) }\end{array}$ & - & - & - & - & - & - & - & - & - & - & - & - & - & - & - & - & - & - & - & - \\
\hline & Bairdia piscariformis Chen, 1958 & - & - & - & - & - & - & - & - & - & - & - & - & - & - & - & - & - & - & - & - \\
\hline & Bairdia lungtanensis Chen, 1958 & - & - & - & - & - & - & - & - & - & - & - & - & - & - & - & - & - & - & - & - \\
\hline & Basslerella tota Chen \& Bao, 1986 & - & - & - & - & - & - & - & - & - & - & - & - & - & - & 曰 & - & - & - & - & - \\
\hline & Bairdia piscariformis Chen, 1958 & - & - & - & - & - & - & - & - & - & - & - & - & - & - & - & - & - & - & - & - \\
\hline & Bairdia trianguliformis Chen, 1958 & - & - & - & - & - & - & - & - & - & - & - & - & - & घ & - & - & - & - & - & - \\
\hline & Bairdiacypris longirobusta Chen, 1958 & - & - & - & - & - & - & - & - & - & - & - & - & - & - & - & - & - & - & - & - \\
\hline & Cryptobairdia seminalis (Knight, 1928) & - & - & - & - & - & - & - & - & - & - & - & - & - & - & - & - & - & - & - & - \\
\hline & Bairdiacypris deloi Bradfield, 1935 & - & - & - & - & - & - & - & - & - & - & - & - & - & - & - & - & - & - & - & - \\
\hline \multicolumn{2}{|c|}{ Number of species in the area where they are less: $n 1$} & na & na & 23 & 29 & na & 63 & 130 & 35 & 66 & 22 & 14 & 60 & 87 & 94 & 53 & 62 & 38 & 98 & 112 & 130 \\
\hline \multicolumn{2}{|c|}{ number of common species: C } & 1 & 1 & 1 & 11 & 2 & 4 & 2 & 2 & 4 & 1 & 2 & 8 & 9 & 9 & 2 & 3 & 2 & 11 & 1 & 3 \\
\hline \multicolumn{2}{|l|}{$\mathrm{SI}=\mathrm{C} / \mathrm{n} 1$} & na & na & 0.04 & 0.38 & na & $0.06 c$ & 0.02 & 0.06 & 0.06 & 0.05 & 0.140 & $0.13 c$ & 0.10 & 0.10 & 0.04 & $0.05 c$ & $0.05 c$ & $0.11 \mathrm{C}$ & $0.01 \mathrm{C}$ & 0.02 \\
\hline
\end{tabular}

OCCURRENCES. - Sample 07PB06-5, Ban Naen Sawan II section, Tak Fa Formation, Phetchabun Province, central Thailand, Middle Permian.

\section{REMARKS}

Polycope sp. D is attributed to the genus Polycope because of its subcircular carapace with fine ornamentation on carapace surface.

\section{PALAEOBIOGEOGRAPHIC RELATIONS}

Of the 130 ostracods species reported from the Early and Middle Permian of the studied localities (this work; Chitna- rin et al. 2012), 31 have previously been documented from other localities such as South China (Chen 1958; Guan et al. 1978; Chen \& Shi 1982; Chen \& Bao 1986; Shi \& Chen 1987; Shi \& Chen 2002; Crasquin et al. 2010a; Forel 2012; Zazzali et al. 2015 ), Hungary (Kozur 1985b), Greece (Crasquin-Soleau \& Baud 1998), Italy (Crasquin et al. 2008a), Oman (Crasquin-Soleau et al. 1999), Tunisia (Lethiers et al. 1989), Japan (Ishizaki 1964), Tibet (Crasquin-Soleau et al. 2007), Serbia (Crasquin et al. 2010b), as shown in Table 3. These areas were located within the Palaeo-Tethys during the Early to Late Permian interval (Scotese \& Langford 1995; Crasquin-Soleau et al. 2001; Fig. 30). The encountered genera are quite well known from the Permian shallow platforms (Pe- 


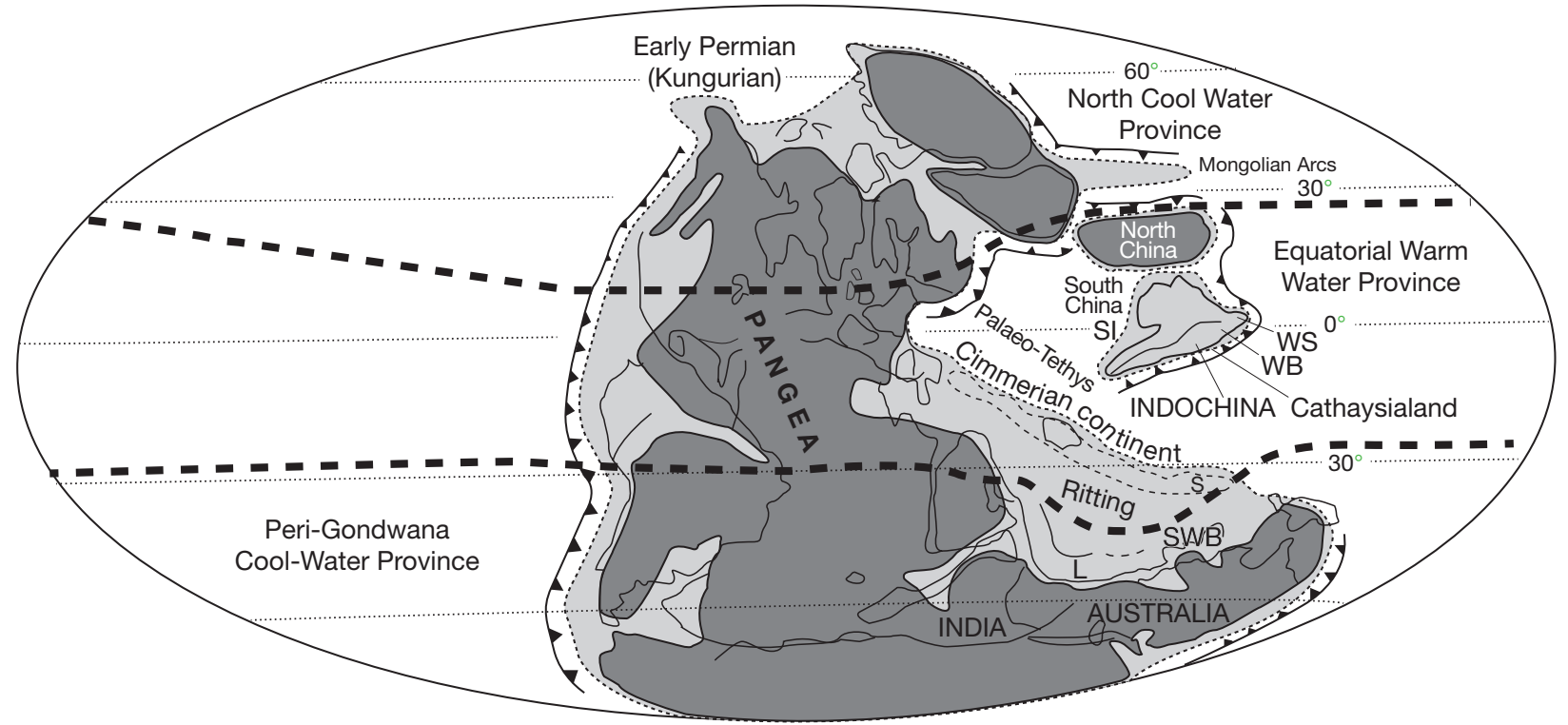

FIG. 30. - Palaeogeographic reconstructions of the Tethyan region with localities of Indochina, Pangea, and Sibumasu (Western Cimmerian Continent) for Late Early Permian (Kungurian) after Metcalfe (2011).

terson \& Kaesler 1980; Costanzo \& Kaesler 1987; Melnyk \& Maddocks 1988; Crasquin-Soleau et al. 1999). Outside the Palaeo-Tethys, Bairdiacypris deloi and Cryptobairdia seminalis are known from Late Carboniferous to Early Permian of the United States of America (Knight 1928; Kellett 1934; Bradfield 1935; Cooper 1946; Sohn 1960).

The Simpson Index (SI) is adopted here as a provincialism index, which can be calculated by the following equation: $\mathrm{SI}=\mathrm{C} / \mathrm{n}_{1}$, where $\mathrm{C}$ is number of common species between two localities and $n_{1}$ is the number of species in the area of lower diversity. The calculated indices are shown in Table 3. As a whole, Thai ostracods have close relationships with the studies of Early Permian in Eastern China (Chen 1958) as illustrated with the highest SI value (0.38). Relationships are recorded during the Middle to Late Permian with Tunisia, South China, and Eastern China (Lethiers et al. 1989; Chen \& Shi 1982; Shi \& Chen 1987; Shi \& Chen 2002; Crasquin et al. 2010a). The relationships with USA is low. Thus, the Indochina and South China blocks were very closed to each other since the Early to Late Permian (Fig. 30).

Most of the studied sections in Thailand are assigned to the Early Permian; therefore, $80 \%$ of the recovered species are recognized from Early Permian onward. In the literature, Chen (1958), Guan et al. (1978) and Chen \& Bao (1986) provide information on the Early Permian ostracods, while others provide information regarding the Middle to Late Permian. These informations are summarized in the Table 3 where the ostracod species ( $2^{\text {nd }}$ and $3^{\text {rd }}$ columns) are grouped by age: numbers 1-3 are of Middle Permian age and numbers 4 to 32 refer to Early Permian data. The columns 4 to 6 correspond to species occurrences of Late Carboniferous to Early Permian age from USA; the column 7 to 9 correlate to Early Permian age in the literature; the column 10 to 23 gather Middle to Late Permian occurrences from the literatures.
The oldest known of the studied ostracods is Bairdiacypris deloi which was reported for the first time from the Late Carboniferous of Western USA; it was later reported from the Middle Permian strata of Greece, Japan and Tunisia. It is also found here in the MIddle Permian limestone of the Tak Fa Formation (sample 07PB05-6). Cryptobairdia seminalis the Late Carboniferous to Early Permian fauna of USA is recovered from Early Permian of the Tak Fa Formation (samples 07PB03-5, 07PB03-7, 07PB04-2), and from Latest Permian of Jiangsu province in China. The presence of these fauna shows connection between Panthlassa Ocean and Palaeo-Tethys at least during the Early Permian (Fig. 30).

Twenty-seven species which originated on the Western shelf of Indochina block during the Early Permian (number 4 to 30 in $2^{\text {nd }}$ and $3^{\text {rd }}$ columns) later dispersed to other parts of the Palaeo-Tethys in the Middle to Late Permian. For example, Bairdia trianguliformis Chen, 1958 known from the early Early Permian (Asselian) of the Nam Maholan Formation in Loei province and the Tak Fa Formation (Artinskian) in Nakhon Sawan province, later occurs in the latest Permian of Guangxi, South China (Chen 1958). Samarella viscusforma Chitnarin, 2012 recovered from the Early Permian strata of the present analysis was found in the latest Permian strata of Sichuan Province, South China (Zazzali et al. 2015). Sulcella mesopermiana Kozur, 1985, S. suprapermiana Kozur, 1985 and Bairdia deducta deduta Zalanyi, 1974 sensu Kozur (1985a) which have been reported from the Middle to Late Permian of Hungary, Oman, Greece, Italy and Serbia (Kozur 1985a, b; Crasquin-Soleau \& Baud 1998; Crasquin-Soleau et al. 1999; Crasquin et al. 2008b; Crasquin et al. 2010b), are recovered from the Early Permian of Thailand. This confirms the connection between Eastern (Thailand-Indochina block) and Western rims of the Palaeo-Tethys. On another hand, two species Microcheilinella shicheni Crasquin, 2010 
and Bairdia guangxiensis Guan, 1978 reported from the Early Permian of Eastern China (Chen 1958; Guan et al. 1978) are recovered from the Middle Permian strata here. This also shows the relationship between Indochina and South China Blocks during the Early Permian.

\section{CONCLUSIONS}

The Early to Middle Permian ostracods have been recovered from limestones collected from 12 sections of central Thailand (the Indochina Block). At least, 130 species have been identified. We presented an earlier article focusing on Order Palaeocopida (Chitnarin et al. 2012) where 39 species were reported, eight were newly described. We publish here Podocopida, Platycopida and Myodocopida. There are 91 species described in this article, 14 are newly described. These ostracods represent the shallow to slightly deep condition on the carbonate platform of the Indochina Block. They have close relationships to South China from the Early to Late Permian.

\section{Acknowledgements}

This research was funded by grant fund under the program Strategic Scholarships for Frontier Research Network for the PhD Program Thai Doctoral degree from the Office of the Higher Education Commission, Thailand (The CHEPhD-THA Scholarship) and French Embassy in Thailand. The authors are very gratefull to Dr Ewa Olempska (Instytut Paleobiologii PAN, Warzaw, Poland) and to two anonymous reviewers for the accurate examination and review of the manuscript.

\section{REFERENCES}

Altermann W. 1989. - Facies Development in the Permian Phetchabun Basin Central Thailand. VWBVerlag fur Wissenschaft und Bildung, Berlin, 275 p.

Assama K., IWa i I., Veeraburus M. \& Hongnusonthi A. 1968. - Permian plants from Loei, Thailand. Journal of Geology and Paleontology of Southeast Asia 4: 82-99.

AssaWapatchara S. 2001. - Geology of Ta Kli on Scale 1:50000 Sheet Ta Kli (5039 I), Changwat Nakhon Sawan. Department of Mineral Resources, Bangkok.

BeCKER G. 2002. - Palaeozoic Ostracoda: the standard classification scheme. Neues Jahrbuch für Geologie und Paläontologie 266: 165-228.

Bradfield H. H. 1935. - Pennsylvanian Ostracoda of Ardmore Basin, Oklahoma. Bulletins of American Paleontology 22: 1-145.

BRADY G. S. 1867. - A Synopsis of the Recent British Ostracoda. The Intellectual Observer 12 (2): 110-130, 2 pls.

Burrett B., UdChachon M., ThassanapaK H. \& Chitnarin A. 2014. - Conodonts, radiolarians and ostracodes in the Permian E-Lert Formation, Loei Fold Belt, Indochina Terrane, Thailand. Geological Magazine 152 (1): 106-142 (published online 16 May 2014). https://doi.org/10.1017/S001675681400017X

Charoenprawat A. \& Wongwanich T. 1976. - Preliminary Geology of Changwat Loei, Map Sheet (ND 47-12). Geological Survey Division, Department of Mineral Resources, Bangkok.

Chen D. \& BAO H. 1986. - Lower Permian ostracodes from the Chihsia Formation of Jurong and Longtan, Jiangsu province. Acta Micropalaeontologica Sinica 3 (2): 107-132.
Chen D. \& SHI C. 1982. — Latest Permian ostracoda from Nantong, Jiangsu and from Miannyang, Hubei. Bulletin of Nanjing Institute of Geology and Palaeontology Academia Sinica 4: 105-152.

CHEN T. C. 1958. - Permian ostracods from the Chihsia limestone of Lungtan, Nanking. Acta Palaeontologica Sinica 6: 215-257.

Chitnarin A., Crasquin S., Charoentitirat T., Tepnarong P. \& Thanee N. 2012. — Ostracods (Crustacea) of the EarlyMiddle Permian from Central Thailand (Indochina block). Part I. Order Palaeocopida. Geodiversitas 34 (4): 801-835. https://doi. org/10.5252/g2012n4a5

Chitnarin A., Crasquin S. Chonglakmani C., Broutin J., Grote P. J. \& Thanee N. 2008. — Middle Permian ostracods from Tak Fa limestone, Phetchabun province, central Thailand. Geobios 41: 341-353. https://doi.org/10.1016/j.geobios.2007.09.001

ChOnglakmani C. \& SatTaYAlaK N. 1979. - Geological Map of Thailand on 1: 250000 Scale: Sheet Changwat Phetchabun (NE4716). Department of Mineral Resources, Bangkok.

Chonglakmani C. \& Fontaine H. 1990. — The Lam Narai-Phetchabun region: a platform of Early Carboniferous to Late Permian age, in Charusiri P., Pisutha-ARnONd V. \& JarupongSAKUl $S$. (eds), Proceedings of the Development Geology of Thailand into the Year 2000. Chulalongkorn University, Bangkok: 39-98.

Cooper C. L. 1946. - Pennsylvanian ostracodes of Illinois. Illinois State Geological Survey Bulletin 70: 1-177. https://hdl.handle. net/2027/uiuo.ark:/13960/t9h42xw6s

Cordell R. J. 1952. — Ostracodes from the Upper Pennsylvanian of Missouri. Part I: the family Bairdiidae. Journal of Paleontology 26: 74-112. http://www.jstor.org/stable/1299773

Coryell H. N. 1928. — Some new Pennsylvanian Ostracoda. Journal of Paleontology 2: 87-94. http://www.jstor.org/stable/1298021

Coryell H. N. \& BoоTH R. T. 1933. - Pennsylvanian Ostracoda; a continuation of the study of the Ostracoda Fauna from the Wayland Shale, Graham, Texas. American Midland Naturalist 14: 258-287. https://doi.org/10.2307/2420189

Coryell H. N. \& SAmple C. H. 1932. - Pennsylvanian Ostracoda, a study of the ostracode fauna of the East Mountain Shale, Mineral Wells, Texas. American Midland Naturalist 13: 245-281. https://doi.org/10.2307/2420173

Costanzo G. V. \& Kaesler R. L. 1987. — Changes in Permian marine Ostracode faunas during regression, Florena Shale, Northeastern Kansas. Journal of Paleontology 61: 1204-1215. https:// doi.org/10.1017/S0022336000029577

Crasquin-Soleau S. \& Baud A. 1998. - New Permian ostracods from Greece (Hydra Island). Journal of Micropalaeontology 17: 131-152. https://doi.org/10.1144/jm.17.2.131

Crasquin-Soleau S., Broutin J., Roger J., Plate, J.P., Al Hashmi H., Angiolini L., Baud A., Bucher H., \& Marcoux J. 1999. First Permian Ostracode Fauna from the Arabian Plate (Khuff Formation, Sultanate of Oman). Micropaleontology 45: 163-183. https://doi.org/10.2307/1486111

Crasquin-Soleau S., Boutin J., Besse J. \& Berthelin M. 2001. Ostracods and palaeobotany from the middle Permian of Oman: implications on Pangaea reconstruction. Terra Nova 13: 38-43. https://10.1046/j.1365-3121.2001.00311.x

Crasquin-Soleau S., Vaslet D. \& Le Nindre Y. M. 2005. Ostracods from Permian-Triassic boundary in Saudi Arabia (Khuff Formation). Palaeontology 48: 853-868. https://doi. org/10.1111/j.1475-4983.2005.00476.x

Crasquin-Soleau S., Marcoux J., Angiolini L., Richoz S., Nicron A., Baud A. \& Bertho Y. 2004. - A new ostracodes fauna from the Permian-Triassic boundary in Turkey (taurua, Antalya Nappes). Micropaleontology 50 (3): 281-295. https:// doi.org/10.2113/50.3.281

Crasquin-Soleau S., Galfetti T., Bucher H. \& Brayard A. 2006. - Early Triassic ostracods from Guangxi Province, South China. Rivista Italiana di Paleontologia e Stratigraia 112: 55-75. Crasquin-Soleau S., Galfetti T., Bucher H., Kershaw S. \& FENG Q. 2007. - Ostracod fauna recovery in the aftermath of 
Permian-Triassic crisis: Palaeozoic-Mesozoic turnover. Hydrobiologia 585: 13-27. https://doi.org/10.1007/978-1-4020-6418-0_2

Crasquin S., Carcione L. \& Martini R. 2008a. - Permian ostracods from the Lercara Formation (Middle Triassic-Carnian?), Silcily, Italy. Palaeontology 51 (3): 537-560. https://doi. org/10.1111/j.1475-4983.2008.00755.x

Crasquin S., Perri M. C., Nicora A. \& De Wever P. 2008b. Ostracods across the Permian-Triassic boundary in western Tethys: the Bulla Parastratotype (Southern Alps, Italy). Rivista Italiana di Paleontologia e Stratigrafia 114: 233-262.

Crasquin S., Forel M. B., Feng Q., YUAN A., Baudin F. \& Collin P. Y. 2010a. - Ostracods (Crustacea) through Permian-Triassic boundary in South China: the Meishan stratotype (Zhejiang Province). Journal of Systematic Palaeontology 8: 331-370. https:// doi.org/10.1080/14772011003784992

Crasquin S., Sudar M. N., Jovanović D. \& Kolar-Jurkovšek T. 2010b - Upper Permian ostracode assemblage from the Jadar Block (Vardar Zone, NW Serbia). Geološki anali Balkanskoga polistrva 71: 23-25.

Croneis C. \& Thurman F. A. 1939. - New Ostracodes from the Kinkaid Formation. Journal of the Scientific Laboratories, Denison University 33: 297-330.

DAWSON O. \& RACEY A. 1993. - Fusuline-calcareous algal biofacies of the Permian Ratburi Limestone, Saraburi, Central Thailand. Journal of Southeast Asian Earth Sciences 8: 49-65. https://doi. org/10.1016/0743-9547(93)90007-C

Delo D. M. 1930. - Some Upper Carboniferous Ostracoda from the Shale Basin of Western Texas. Journal of Paleontology 4: 152178. http://www.jstor.org/stable/1297872

Fontaine H., Salyapongse S. \& Vachard D. 1999. - New carboniferous fossils found in Ban Bo Nam Area, Central Thailand, in Khantaprab C. (ed.), Proceedings of the Symposium on Mineral, Energy, and Water Resources of Thailand: Towards the Year 2000. Bangkok: 201-211.

Fontaine H., Salyaponges S. Tien D. N. \& Vachard D. 2002. The Permian of Khao Tham Yai area in Northeast Thailand, in Nopadon Mantajit (ed.), Proceedings of the Symposium on Geology of Thailand. Bangkok: 45-57.

FOREL M. B. 2012. - Ostracods (Crustacea) associated with microbialites across the Permian-Triassic boundary in Dajiang (Guizhou Province, south China). European Journal of Taxonomy 19: 1-34. https://doi.org/10.5852/ejt.2012.19

GEIS H. L. 1933. - Microcheilinella, a new name for the ostracode genus Microcheilus. Journal of Paleontology 7: 112.

GRÜNDEL J. 1962. — Zur Taxonomie der Ostracoden der Gattendorfia-Stufe Thüringens. Freiberger Forschungshefte 151: 51-105.

Guan S., Sun Q., Jiang Y., Li L., Zhao B., Zhang X., Yang R. \& FENG B. 1978. - Subclass Ostracoda, in Paleontological Atlas of Central and South China. Geological Publishing House, Beijing: 115-325 (in Chinese).

Hada S., Khosithanont S., Goto H., Fontaine H. \& Salyapongse S. 2015. - Evolution and extinction of Permian fusulinid fauna in the Khao Tham Yai Limestone in NE Thailand. Journal of Asian Earth Sciences 104: 175-184.

Hamilton I. B. 1942. - Ostracodes from the Upper Permian of Texas. Journal of Paleontology 16 (6): 712-718. http://www.jstor. org/stable/1298975

Harlton B. H. 1928. - Pennsylvanian ostracodes from Oklahoma and Texas. Journal of Paleontology 2 (2): 132-141, pl. 21. http:// www.jstor.org/stable/1297837

HarLton B. H. 1929a. - Some Upper Mississippian (Fayetteville) and Lower Pennsylvanian (Wapanucka-Morrow) Ostracoda of Oklahoma and Arkansas. American Journal of Sciences 18 (105): 254-270.

Harlton B. H. 1929b. - Pennsylvanian Ostracoda from Menard County, Texas. Texas University Bulletin 2901: 139-161.

Horne D. J., Cohen A. \& Martens K. 2002. - Taxonomy, Morphology and Biology of Quaternary and living Ostracoda in Holmes J. A. \& A. Chivas A. (ed.), The Ostracoda: applications in Quaternary Research. Geophysical Monograph 131: 5-36.

IgO H. 1972. - Fusulinacean fossils from Thailand, Part VI. Fusulinacean fossils from North Thailand, in KOBAYASHI T. \& ToriYama R. (eds), Geology and Palaeontology of Southeast Asia 10: 63-116.

Ishibashi T., FujIYAma M. \& NAKORNSRi N. 1996. - Permian Ammonoids from the Loei area, Northeast Thailand. Earth and Planetary Science. Memoir of Faculty of Science, Kyoshu University: $169-175$

IsHIZAKI K. 1964. - Middle Permian ostracodes from the Iwaizaki Limestone, northeast Japan. Science Reports of the Tohoku University, Second Series, Geology 36: 139-160.

KelletT B. 1934. - Ostracodes of the Upper Pennsylvanian and the Lower Permian Strata of Kansas II. The Genus Bairdia. Journal of Paleontology 8 (2): 120-138. http://www.jstor.org/stable/1298077

Kellett B. 1935. - Ostracodes of the Upper Pennsylvanian and the Lower Permian strata of Kansas: III. Bairdiidae (concluded), Cytherellidae, Cypridinidae, Entomoconchidae, Cytheridae and Cypridae. Journal of Paleontology 9: 132-166. http://www.jstor. org/stable/1298216

Ketwetsuriya C., Nützel A. \& Kanjanapayont P. 2016. - Permian gastropods from the Tak Fa Limestone, Nakhon Sawan, Northern Thailand. Bulletin of Geosciences 19 (3): 481-513. https://doi.org/10.3140/bull.geosci.1617

KNIGHT J. 1928. - Some Pennsylvanian ostracodes from the Henrietta Formation of eastern Missouri. Journal of Paleontology 2: 229-267, 318-337. http://www.jstor.org/stable/1298017

KozUR H. 1985a. - Biostratigraphic evaluation of the Upper Paleozoic conodonts, ostracods and holothurian sclerites of the Bükk Mts. Part II: Upper Paleozoic ostracods. Acta Geologica Hungarica 28 (34): 225-256.

Kozur H. 1985b. - Neue Ostracoden - Arten aus dem Oberen Mittelkarbon (Höberes Moskovian), Mittel- und Oberperm des Bükk-gebirges (N-Ungarn). Geologish Paläontologische Mitteilungen Innsbruck 2: 1-145.

Kozur H., McKenzie K. G. \& Mostler H. 1996. - Microcheilinella Geis (ostracoda) with preserved soft parts from Upper Triassic Hallstatt Limestonees of the Alps, with remarks on the relationsand systematic position of Bairdiacypridacea and Sigillicea, in KeEn M. (ed.), Proceedings of the Second European Ostracodologist Meeting. British Micropalaeontological Society, London: 9-16.

KuMmerow E. 1953. - Ueber Oberkarbonische und Devonische Ostracoden in Deutschland und in der Volksrepublik Polen. Geologie, Beiheft 7, 75 p.

Lethiers F. \& Crasquin-Soleau S. 1988. - Comment extraire des microfossiles à tests calcitiques de roches calcaires dures. Revue de Micropaléontologie 31: 56-61.

Lethiers F., Razagalliah S., Colin J.-P. \& Vachard D. 1989. Micropalaeontology of the Permian marls of Merbah el Oussif (Jebel Tebaga, Tunisia) with special emphasis on the ostracods. Journal of Micropaleontology 8: 227-238. https://doi.org/10.1144/ jm.8.2.227

NAKORNSRI N. 1981. - Geology and Mineral Resources of Map Sheet Amphoe Ban Mi. Department of Mineral Resources, Bangkok.

MELNYK D. \& MADDOCKS R. 1988. — Ostracode biostratigraphy of the Permo Carboniferous of central and north central Texas, part I: Paleoenvironmental framework. Micropaleontology 34 (1): 1-20. https://doi.org/10.2307/1485607

MetCalfe I. 2011. - Tectonic framework and Phanerozoic evolution of Sundaland. Gondwana Research 19: 3-21. https://doi org/10.1016/j.gr.2010.02.016

MetCAlfe I. \& Sone M. 2008. - Biostratigraphy and palaeobiogeography of Lower Permian (lower Kungurian) conodonts from the Tak Fa Formation (Saraburi Limestone), Thailand. Palaeogeography, Palaeoclimatology, Palaeoecology 257: 139-151. https://doi.org/10.1016/j.palaeo.2007.09.014

MoORE R. C. 1961. — Treatise of Invertebrate Paleontology, part Q, 
Arthropoda 3. Geological Society of America, University of Kansas Press, Lawrence, $442 \mathrm{p}$.

Pérez-Huerta A., Chonglakmani C. \& Chitnarin A. 2007. Permian brachiopods from new localities in northeast Thailand: Implications for paleobiogeographic analysis. Journal of Asian Earth Science 30: 504-517. https://doi.org/10.1016/j.jseaes.2006.11.012

Peterson R. M. \& KAesLer R. L. 1980. — Distribution and diversity of ostracodes assemblages from the Hamlin Shale and the Americus limestones (Permian, Wolfcampian) in northern Kansas. University of Kansas Paleontological Contributions 100: 1-26.

Posner V. W. 1951. - Lower Carboniferous ostracodes from the western Flank of the Moscow Basin. Trudy Vsesoyuznogo Neftyanogo Nauchno-Issledovatel'skogo Geologo-Razvedochnogo Instituta 56: 5-108.

ROTH R. \& SKINNER J. 1930. - The fauna of the McCoy Formation, Pennsylvanian of Colorado. Journal of Paleontology 4: 332-352. http://www.jstor.org/stable/1298000

ROZHDESTVENSKAJA A. A. 1959. - [ O Ostracodes from the terrigenous Devonian strata of Western Bashkiria and their stratigraphic significance]. Akademia Nauk SSSR Bashkirkskii fiflial, RISO AN CCCP, 111 (33B): 117-247 (in Russian).

SCOTESE C. R. \& LANGFord R. P. 1995. - Pangea and the Paleogeography of the Permian, in SCHOlle P. A., PERYT T. M. \& UlMERScholle D. S. (eds), The Permian of Northern Pangea. Springer, Berlin: 3-19. https://doi.org/10.1007/978-3-642-78593-1_1

SHI C. G. \& CHEN D. Q. 1987. - The Changhsingian ostracodes from Meishan, Chanxing, Zhejiang. Stratigraphy and palaeontology of Systemic boundary in China, Permian and Triassic Boundary 5: 23-80.

SHI C. G. \& CHEN D. Q. 2002. — Late Permian ostracodes from Heshan and Yishan of Guangxi. Bulletin of Nanjing Institute Geology and Paleontology, Academia Sinica 15: 47-129 (in Chinese with English abstract).

SoHN I. 1960. - Paleozoic species of Bairdia and related genera. Geological Survey Professional Paper 330-A: 1-105.

Sone M., Chonglakmani C. \& Chitnarin A. 2009. — Middle Permian Producidine brachiopods from central Thailand (the Indochina Terrane) with paleobiogeographic implications. Journal of Paleontology 83: 804-810. https://doi.org/10.1666/09-001.1

UdChachon M., Chonglakmani C., Campbell, H. \& Thanee N. 2007. — Late Middle Permian Alatoconchid-bearing limestones from the south of the Khao Khwang Platform, Central Thailand, in TANTIWANIT W. (ed.), Proceedings of International Confer- ence on GEOTHAI'2007. Department of Mineral Resources, Bangkok: 169-176.

Ulrich E. O. \& BASSLER R. S. 1906. — New American Palaeozoic ostracoda. Notes and descriptions of the Upper Carboniferous genera and species. U.S. National Museum Paper 30: 149-164. https://doi.org/10.5479/si.00963801.30-1446.149

Ulrich E. O. \& Bassler R. S. 1923. - Paleozoic Ostracoda: Their Morphology, Classification and Occurrence. Maryland Geological Survey, Silurian 9: 271-391.

WANG S. Q. 1978. - Late Permian and Early Triassic ostracods of Western Guizhou and northeastern Yunnan. Acta Palaeontologica Sinica 17: 277-312 (in Chinese with English abstract).

WielchowsKy C. \& YOUNG J. 1985. - Regional facies variation in Permian of the Phetchabun fold and thrust belt, Thailand, in Thanuvarachon P., Hokja Roen S. \& Yanungme W. (eds), Proceeding of the Conference on Geology and Mineral Resources Development of Northeastern Thailand. Department of Geotechnology, Khon Kaen University, Khon Kaen: 41-55.

YANAGIDA J. \& NAKORNSRI H. 1999. — Permian brachiopods from the Khao Hin Kling area near Phetchabun, North-central Thailand. Bulletin of the Kitakyushu Museum of Natural History and Human History 18: 105-136.

YI W. 1993. - Ostracode fauna from Lower Permian Chihsia Formation of Guangping in Datian, Fujian. Acta Palaeontologica Sinica 32 (5): 561-586.

YI W. 2004. - Ostracodes from the Upper Permian and Lower Triassic at the Kongtongshan section of Datian, Fujian. Acta Palaeontologica Sinica 43: 556-570 (in Chinese with English abstract).

Yuan A., Crasquin S., Feng Q. \& Gu S. 2009. — Ostracods from the uppermost Permian siliceous and muddy rocks of Guizhou, Guangxi and Anhui. Acta Micropalaeontologica Sinica 26: 385-403.

ZALÁNYI B. 1974. - Die oberpermischen ostracoden des Bükkgebirges, in Sidó M., ZALÁNYI B. \& SCHrÉTER Z. (eds), Neue paläontologische Ergebnisse aus dem Oberpaläozoikum des Bükk-Gebirgrs. Akadémiai Kiado, Budapest: 95-251.

Zazzali S., CRasquin S., Deconinck J. F. \& Feng Q. 2015. Biodiversity across the Guadalupian-Lopingian Boundary: first results on the ostracod (Crustacea) fauna, Chaotian section (Sichuan Province, South China). Geodiversitas 37 (3): 283-313. https://doi.org/10.5252/g2015n3a1

ZHENG S. 1976. - Early Mesozoic ostracodes from some localities in SouthWest China. Acta Palaeontologica Sinica 15: 77-93 (in Chinese with English abstract). 